Pacific

Journal of

Mathematics

CRITICAL-EXPONENT SOBOLEV NORMS AND THE SLICE THEOREM FOR THE QUOTIENT SPACE OF CONNECTIONS

Paul M.N. Feehan

Volume $200 \quad$ No. 1

September 2001 


\title{
CRITICAL-EXPONENT SOBOLEV NORMS AND THE SLICE THEOREM FOR THE QUOTIENT SPACE OF CONNECTIONS
}

\author{
PAUl M.N. FEEHAN
}

\begin{abstract}
Following Taubes, we describe a collection of critical-exponent Sobolev norms, discuss their embedding and multiplication properties, and describe optimal Green's operator estimates where the constants depend at most on the first positive eigenvalue of the covariant Laplacian of a $G$ connection and the $L^{2}$ norm of the connection's curvature, for arbitrary compact Lie groups $G$. Using these critical-exponent norms, we prove a sharp, global analogue of Uhlenbeck's Coulomb gaugefixing theorem, where the usual product connection over a ball is replaced by an arbitrary reference connection over the entire manifold. We also prove a quantitative version of the conventional slice theorem for the quotient space of $G$ connections, with an invariant and sharp characterization of those points in the quotient space which are contained in the image of an $L^{4}$ ball in the Coulomb-gauge slice. Our gauge-fixing and slice theorems use $L_{1}^{2}$ distance functions on the quotient space and the estimate constants depend at most on the first positive eigenvalue of the covariant Laplacian of the reference connection and the $L^{2}$ norm of its curvature.
\end{abstract}

\section{Introduction.}

The use of certain "critical-exponent" Sobolev norms is an important feature of methods employed by Taubes to solve the anti-self-dual and related non-linear elliptic partial differential equations $[\mathbf{2 3}],[24],[25]$. Indeed, the estimates one can obtain using these critical-exponent norms appear to be the best possible when one needs to bound the norm of a Green's operator for a Laplacian, depending on a connection varying in a non-compact family, in terms of minimal data such as the first positive eigenvalue of the Laplacian or the $L^{2}$ norm of the curvature of the connection. Despite their utility, particularly in applications where an optimal analysis is required for gluing or degeneration problems (for example, when considering Uhlenbeck-bubbling families of anti-self-dual connections or PU(2) monopoles), these methods are not widely known. Following Taubes [21], [23], [24], [25] we describe a collection of critical-exponent Sobolev norms and general Green's operator 
estimates depending only on first positive eigenvalues or the $L^{2}$ norm of the connection's curvature. These estimates are especially useful both for the construction of gluing maps, in the case of either anti-self-dual connections [24] or, more recently, in the case of $\mathrm{PU}(2)$ monopoles [6], [7], [9] and for analyzing their asymptotic behavior with respect to Uhlenbeck limits of the underlying gluing data. We apply them here to prove an optimal slice theorem for the quotient space of connections. The result is "optimal" in the sense that if a point $[A]$ in the quotient space is known to be just $L_{1}^{2}$-close enough to a reference point $\left[A_{0}\right]$ (see below for the precise statement), then $A$ can be placed in Coulomb gauge relative to $A_{0}$, with all constants depending at most on the first positive eigenvalue of the covariant Laplacian defined by $A_{0}$ and the $L^{2}$ norm of the curvature of $A_{0}$. Such slice theorems are particularly advantageous when analyzing gluing maps and their differentials in situations (such as those of $[\mathbf{8}],[\mathbf{9}]$ ) where the underlying gluing data is allowed to "bubble". In this paper we shall for simplicity only consider connections over four-dimensional manifolds, but the methods and results can adapted to the case of manifolds of arbitrary dimension, as in [26], to prove slice theorems applicable to cases where the reference connection is allowed to degenerate.

\subsection{Critical-exponent Sobolev norms and the slice theorem. Sup-} pose that $X$ is a closed, Riemannian four-manifold, that $G$ is a compact Lie group, and that $\mathcal{B}_{E}^{k, p}=\mathcal{A}_{E}^{k, p} / \mathcal{G}_{E}^{k+1, p}$ is the quotient space of $L_{k}^{p}$ connections on a $G$ bundle $E$ modulo the Banach Lie group of $L_{k+1}^{p}$ gauge transformations. Here, the integer $k \geq 1$ and the Sobolev exponent $1<p<\infty$ obey the constraint $(k+1) p>4$, so $L_{k+1}^{p}(X) \subset C^{0}(X)$ and gauge transformations in $\mathcal{G}_{E}^{k+1, p}$ are continuous. When $(k+1) p=4$ we have the "borderline", "critical", or "limiting case" of the Sobolev embedding theorem: $L_{k+1}^{p}(X) \subset L^{q}(X)$ for all $q<\infty$ but not $q=\infty$.

A connection $A \in \mathcal{A}_{E}^{k, p}$ is in Coulomb gauge relative to a reference connection $A_{0}$ if $d_{A_{0}}^{*}\left(A-A_{0}\right)=0$ and it is a standard result that $\mathbf{S}_{A_{0}}=$ $A_{0}+\operatorname{Ker} d_{A_{0}}^{*} \subset \mathcal{A}_{E}^{k, p}$ provides a slice for the action of the gauge group $\mathcal{G}_{E}^{k+1, p}[\mathbf{2}],[\mathbf{5}],[\mathbf{1 0}],[\mathbf{1 1}],[\mathbf{1 3}],[\mathbf{1 4}],[\mathbf{1 6}],[\mathbf{1 8}]$. (See Proposition 3.4 for a detailed statement.) More exactly, if $\mathbf{B}_{A_{0}}^{k, p}(\varepsilon)$ is the $L_{k}^{p}$ ball in $\mathbf{S}_{A_{0}}$ with center $A_{0}$ and $L_{k, A_{0}}^{p}$-radius $\varepsilon$ and $\operatorname{Stab}_{A_{0}} \subset \mathcal{G}_{E}^{k+1, p}$ is the stabilizer of $A_{0}$, then the projection $\pi: \mathbf{B}_{A_{0}}^{k, p}(\varepsilon) / \operatorname{Stab}_{A_{0}} \rightarrow \mathcal{B}_{E}^{k, p}$ is a homeomorphism onto its image and thus contains a small enough $L_{k}^{p}$ ball

$$
B_{\left[A_{0}\right]}^{k, p}(\eta)=\left\{[A] \in \mathcal{B}_{E}^{k}: \operatorname{dist}_{L_{k, A_{0}}^{p}}\left([A],\left[A_{0}\right]\right)<\eta\right\}
$$


where gauge-invariant distance functions on the $\mathcal{G}_{E}^{k+1, p}$-quotient are defined by

$$
\operatorname{dist}_{L_{k^{\prime}, A_{0}}^{p^{\prime}}}\left([A],\left[A_{0}\right]\right)=\inf _{u \in \mathcal{G}_{E}^{k+1, p}}\left\|u(A)-A_{0}\right\|_{L_{k^{\prime}+1, A_{0}}^{p^{\prime}}},
$$

whenever $L_{k}^{p} \subset L_{k^{\prime}}^{p^{\prime}}$. One unsatisfactory aspect of the standard slice theorem concerns the dependence of the constants $\varepsilon\left(\left[A_{0}\right], k, p\right)$ and $\eta\left(\left[A_{0}\right], k, p\right)$ above on the orbit $\left[A_{0}\right]$ - in particular on the curvature $F_{A_{0}}$ - when $k$ and $p$ are large enough that gauge transformations in $\mathcal{G}_{E}^{k+1, p}$ are continuous. Even in the minimal cases, $k=1$ and $p>2$ or $k=2$ and $p=2$, the constants $\varepsilon, \eta$ depend unfavorably on $\left[A_{0}\right]$ when the curvature $F_{A_{0}}$ bubbles. This makes it difficult to analyze the asymptotic behavior of Taubes' gluing maps [20], $[\mathbf{2 2}],[\mathbf{2 3}],[\mathbf{2 4}]$ and their differentials on neighborhoods of points in the Uhlenbeck boundary of the moduli space of anti-self-dual connections, since the balls $\mathbf{B}_{A_{0}}^{k, p}(\varepsilon)$ and $B_{\left[A_{0}\right]}^{k, p}(\eta)$ tend to shrink as $\left[A_{0}\right]$ approaches an Uhlenbeckboundary point. For example, if the connection $A_{0}$ is anti-self-dual, then its energy is bounded by a constant depending only on the topology of $E$ via the Chern-Weil identity $[5, \S 2.1 .4]$, whereas $\left\|F_{A_{0}}\right\|_{L^{p}}$ (with $p>2$ ) or $\left\|F_{A_{0}}\right\|_{L_{1, A_{0}}^{2}}$ tends to infinity as the curvature of $A_{0}$ bubbles.

Our main purpose in this article is to prove a global analogue, Theorem 1.1, of Uhlenbeck's local Coulomb gauge-fixing theorem [26, Theorems $1.3 \& 2.1]$ and a corresponding slice theorem, Theorem 1.2, where the radii of the coordinate balls on the quotient $\mathcal{B}_{E}^{k, p}$ depend only on $\left\|F_{A_{0}}\right\|_{L^{2}}$ and the least positive eigenvalue $\nu_{0}\left[A_{0}\right]$ of the Laplacian $d_{A_{0}}^{*} d_{A_{0}}$ on $\Omega^{0}\left(\mathfrak{g}_{E}\right)$. The key difficulty in establishing Theorem 1.1 is to ensure that the constants depend at most on $\left\|F_{A_{0}}\right\|_{L^{2}}$ and $\nu_{0}\left[A_{0}\right]$ : To guarantee this minimal dependence, we employ critical-exponent Sobolev norms (defined below) to circumvent the fact that when $(k+1) p=4$ the standard Sobolev embedding and multiplication theorems fall just short of what one needs to give the quotient $\mathcal{B}_{E}^{k, p}=\mathcal{A}_{E}^{k, p} / \mathcal{G}_{E}^{k+1, p}$ a manifold structure (see Section 4). Such norms were introduced by Taubes for related purposes in [23].

1.2. Statement of results. For clarity, we now fix $p=2$ and $k \geq 2$ and define the following distance functions on the quotient space $\mathcal{B}_{E}^{k}=\mathcal{A}_{E}^{k} / \mathcal{G}_{E}^{k+1}$ of $L_{k}^{2}$ connections modulo $L_{k+1}^{2}$ gauge transformations,

$$
\begin{aligned}
& \operatorname{dist}_{\mathcal{L}_{1, A_{0}}^{\sharp, 2}}\left([A],\left[A_{0}\right]\right)=\inf _{u \in \mathcal{G}_{E}^{k+1}}\left(\left\|u(A)-A_{0}\right\|_{L^{2 \sharp, 4}}+\left\|d_{A_{0}}^{*}\left(u(A)-A_{0}\right)\right\|_{L^{\sharp, 2}}\right), \\
& \operatorname{dist}_{L_{1, A_{0}}^{\sharp, 2}}\left([A],\left[A_{0}\right]\right)=\inf _{u \in \mathcal{G}_{E}^{k+1}}\left(\left\|u(A)-A_{0}\right\|_{L_{1, A_{0}}^{2}}+\left\|d_{A_{0}}^{*}\left(u(A)-A_{0}\right)\right\|_{L^{\sharp, 2}}\right),
\end{aligned}
$$


where the norms of $a \in \Omega^{1}\left(\mathfrak{g}_{E}\right)$ are defined by (see Equations (4.1), (4.2), and (4.3)),

$$
\begin{aligned}
\|a\|_{L^{\sharp}(X)} & =\sup _{x \in X}\left\|\operatorname{dist}^{-2}(x, \cdot)|a|\right\|_{L^{1}(X)}, \\
\|a\|_{L^{2 \sharp}(X)} & =\sup _{x \in X}\left\|\operatorname{dist}^{-1}(x, \cdot)|a|\right\|_{L^{2}(X)}, \\
\|a\|_{L^{\sharp, 2}(X)} & =\|a\|_{L^{2}(X)}+\|a\|_{L^{\sharp}(X)}, \\
\|a\|_{L^{2 \sharp, 4}(X)} & =\|a\|_{L^{4}(X)}+\|a\|_{L^{2 \sharp}(X)}, \\
\|a\|_{L_{1, A_{0}}^{2}(X)} & =\left(\|a\|_{L^{2}(X)}^{2}+\left\|\nabla_{A_{0}} a\right\|_{L^{2}(X)}^{2}\right)^{1 / 2} .
\end{aligned}
$$

Here, $\operatorname{dist}(x, y)$ denotes the geodesic distance between points $x, y \in X$. The distance function $\operatorname{dist}_{L_{1, A_{0}}^{\sharp, 2}}\left([A],\left[A_{0}\right]\right)$ is bounded by scale invariant norms, $\|a\|_{L^{4}(X, g)}+\left\|\nabla_{A}^{g} a\right\|_{L^{2}(X, g)}+\sup _{x \in X}\left\|\operatorname{dist}_{g}^{-2}(x, \cdot)\left|d_{A_{0}}^{* g} a\right|\right\|_{L^{1}(X, g)}, \quad a \in \Omega^{1}\left(\mathfrak{g}_{E}\right)$, since the $L^{4 / \ell}$ norm on $\otimes^{\ell}\left(T^{*} X\right)$ is conformally invariant, while the third term is invariant under constant rescalings $g \mapsto \tilde{g}=\lambda^{-2} g$ of the metric, as $d_{A_{0}}^{* \tilde{g}} a=\lambda^{2} d_{A_{0}}^{*_{g}} a$, dist $\operatorname{dig}_{\tilde{g}}^{-2}(x, y)=\lambda^{2} \operatorname{dist}_{g}^{-2}(x, y)$ and $d V_{\tilde{g}}=\lambda^{-4} d V_{g}$. Similarly for dist ${ }_{\mathcal{L}_{1, A_{0}}^{\sharp, 2}}\left([A],\left[A_{0}\right]\right)$. Like the $L^{4}$ norm, the $L^{2 \sharp}$ norm on one-forms is scaleinvariant. Our first result is the following global analogue of Uhlenbeck's theorem and complements results of Taubes in $[\mathbf{2 3}, \S 6]$ :

Theorem 1.1. Let $X$ be a closed, smooth four-manifold with metric $g$ and let $G$ be a compact Lie group. Then there are positive constants $c, z$ with the following significance. Let $E$ be a $G$ bundle over $X$ and suppose that $k \geq 2$ is an integer. Given a point $\left[A_{0}\right]$ in $\mathcal{B}_{E}^{k}$, let $\nu_{0}\left[A_{0}\right]$ be the least positive eigenvalue of the Laplacian $d_{A_{0}}^{*} d_{A_{0}}$ on $\Omega^{0}\left(\mathfrak{g}_{E}\right)$ and set $K_{0}=(1+$ $\left.\nu_{0}\left[A_{0}\right]^{-1}\right)\left(1+\left\|F_{A_{0}}\right\|_{L^{2}}\right)$. Let $\varepsilon_{1}$ be a constant satisfying $0<\varepsilon_{1} \leq z K_{0}^{-2}(1+$ $\left.\nu_{0}\left[A_{0}\right]^{-1 / 2}\right)^{-1}$. Then:

(1) For any $[A] \in \mathcal{B}_{E}^{k}$ with $\operatorname{dist}_{\mathcal{L}_{1, A_{0}}^{\sharp, 2}}\left([A],\left[A_{0}\right]\right)<\varepsilon_{1}$, there is a gauge transformation $u \in \mathcal{G}_{E}^{k+1}$, unique up to an element of the stabilizer $\operatorname{Stab}_{A_{0}} \subset \mathcal{G}_{E}^{k+1}$, such that:

(a) $d_{A_{0}}^{*}\left(u(A)-A_{0}\right)=0$,

(b) $\left\|u(A)-A_{0}\right\|_{L^{2 \sharp, 4}} \leq c K_{0} \operatorname{dist}_{\mathcal{L}_{1, A_{0}}^{\sharp, 2}}\left([A],\left[A_{0}\right]\right)$.

(2) For any $[A] \in \mathcal{B}_{E}^{k}$ with $\operatorname{dist}_{L_{1, A_{0}}^{\sharp, 2}}\left([A],\left[A_{0}\right]\right)<\varepsilon_{1}$, there is a gauge transformation $u \in \mathcal{G}_{E}^{k+1}$, unique up to an element of the stabilizer $\operatorname{Stab}_{A_{0}} \subset \mathcal{G}_{E}^{k+1}$, such that:

(a) $d_{A_{0}}^{*}\left(u(A)-A_{0}\right)=0$,

(b) $\left\|u(A)-A_{0}\right\|_{L^{2 \sharp, 4}} \leq c K_{0} \operatorname{dist}_{\mathcal{L}_{1, A_{0}}^{\sharp, 2}}\left([A],\left[A_{0}\right]\right)$, 
(c) $\left\|u(A)-A_{0}\right\|_{L_{1, A_{0}}^{2}} \leq c K_{0} \operatorname{dist}_{L_{1, A_{0}}^{\sharp, 2}}\left([A],\left[A_{0}\right]\right)$.

In Theorem 2.1 of $[\mathbf{2 6}]$ the $L^{2}$ norm of the curvature $F_{A}$ of a local connection matrix $A$ over the unit ball in $\mathbb{R}^{4}$ provides a natural (gauge-invariant) measure of the distance from $[A]$ to $[\Gamma]$, where $\Gamma$ is the product connection. Uhlenbeck's theorem guarantees the existence of an $L_{k+1}^{p}$ gauge transformation $u$ taking an $L_{k}^{p}$ connection $A$ on the product bundle over the unit four-ball, with product connection $\Gamma$, to a connection $u(A)$ satisfying $d_{\Gamma}^{*}(u(A)-\Gamma)=0$ and $\|u(A)-\Gamma\|_{L_{1}^{2}} \leq c\left\|F_{A}\right\|_{L^{2}}$; one only requires that $\left\|F_{A}\right\|_{L^{2}}$ be smaller than a universal constant.

We next have the following refinement of the standard slice theorem for the quotient space $\mathcal{B}_{E}^{k}$. The observation that an $L^{4}$-ball in Ker $d_{A_{0}}^{*}$ provides a slice for $\mathcal{G}_{E}^{k+1}$ was pointed out to us Mrowka; that slightly smaller $L^{2 \sharp, 4}$ and $L_{1, A_{0}}^{2}$ balls provide slices follows from the second of our two proofs of Theorem 1.1 in Section 8. For any $\varepsilon>0$, we define open balls

$$
\begin{aligned}
B_{\left[A_{0}\right]}^{1, \sharp, 2}(\varepsilon) & =\left\{[A] \in \mathcal{B}_{E}^{k}: \operatorname{dist}_{L_{1, A_{0}}^{\sharp, 2}}\left([A],\left[A_{0}\right]\right)<\varepsilon\right\} \subset \mathcal{B}_{E}^{k}, \\
B_{\left[A_{0}\right]}^{1, *, 2}(\varepsilon) & =\left\{[A] \in \mathcal{B}_{E}^{k}: \operatorname{dist}_{\mathcal{L}_{1, A_{0}}^{\sharp, 2}}\left([A],\left[A_{0}\right]\right)<\varepsilon\right\} \subset \mathcal{B}_{E}^{k}, \\
\mathbf{B}_{A_{0}}^{4}(\varepsilon) & =\left\{A \in \mathcal{A}_{E}^{k}: d_{A_{0}}^{*}\left(A-A_{0}\right)=0 \text { and }\left\|A-A_{0}\right\|_{L^{4}(X)}<\varepsilon\right\} \\
& \subset \mathbf{S}_{A_{0}},
\end{aligned}
$$

where $\mathbf{S}_{A_{0}}=\left\{A_{0}\right\}+\operatorname{Ker}\left(\left.d_{A_{0}}^{*}\right|_{L_{k}^{2}}\right) \subset \mathcal{A}_{E}^{k}$ is the slice through $A_{0}$. We let $\bar{B}_{\left[A_{0}\right]}^{1, \sharp, 2}(\varepsilon)$ and $B_{\left[A_{0}\right]}^{1, *, 2}(\varepsilon)$ denote the closed balls.

Theorem 1.2. Let $X$ be a closed, smooth four-manifold with metric $g$ and let $G$ be a compact Lie group. Then there are positive constants $c_{1}, c_{2}, z$ with the following significance. Let $E$ be a $G$ bundle over $X$, let $k \geq 2$ be an integer, and suppose that $\left[A_{0}\right] \in \mathcal{B}_{E}^{k}$. Then:

(1) For $\varepsilon_{0}$ such that $0<\varepsilon_{0}<z\left(1+\nu_{0}\left[A_{0}\right]^{-1 / 2}\right)^{-1}$, the projection $\pi$ : $\mathbf{B}_{A_{0}}^{4}\left(\varepsilon_{0}\right) / \operatorname{Stab}_{A_{0}} \rightarrow \mathcal{B}_{E}^{k}, A \mapsto[A]$, is a homeomorphism onto an open neighborhood of $\left[A_{0}\right] \in \mathcal{B}_{E}^{k}$ and a diffeomorphism on the open subset where $\operatorname{Stab}_{A_{0}} / \operatorname{Center}(G)$ acts freely;

(2) For any constant $\varepsilon_{1}$ satisfying $0<\varepsilon_{1} \leq z K_{0}^{-2}\left(1+\nu_{0}\left[A_{0}\right]^{-1 / 2}\right)^{-1}$ we have the following inclusions of open neighborhoods in $\mathcal{B}_{E}^{k}$ :

$$
B_{\left[A_{0}\right]}^{1, \sharp, 2}\left(\varepsilon_{1}\right) \subset B_{\left[A_{0}\right]}^{1, *, 2}\left(c_{1} \varepsilon_{1}\right) \subset \pi\left(\mathbf{B}_{A_{0}}^{4}\left(c_{2} K_{0} \varepsilon_{1}\right)\right) .
$$

That sharper versions of the standard slice theorem (as in $[\mathbf{5}],[\mathbf{1 0}],[\mathbf{1 1}]$, for example) would hold is suggested by related results of Taubes, namely [21, Lemma A.1] and [23, Lemma 6.5]: For example, they show that if $u$ is an $L_{2}^{2}$ gauge transformation intertwining $L_{1}^{2}$ connections $A_{i}, i=1,2$, 
obeying a slice condition $d_{A_{0}}^{*}\left(A_{i}-A_{0}\right)=0$ defined by an $L_{1}^{2}$ connection $A_{0}$, then $u$ is necessarily in $C^{0}$. Moreover, transition functions relating neighborhoods of the origin in $\operatorname{Ker}\left(\left.d_{A_{0}}^{*}\right|_{L_{1}^{2}}\right)$ and $\operatorname{Ker}\left(\left.d_{A_{0}+a}^{*}\right|_{L_{1}^{2}}\right)$, where $a$ is $L_{1, A_{0}}^{2}$-small, are constructed in [23, Lemma 6.5]; the constants depend only on $\left\|F_{A_{0}}\right\|_{L^{2}}$ and $\nu_{0}\left[A_{0}\right]$. (See $[\mathbf{2 3}, \S 6]$ for detailed statements and related results.) The proof of Theorem 1.1 makes use of methods developed in [21], [23], [24]. To illustrate applications of the methods of Sections 4 and 5 and to point to possible generalizations of the estimates in this article, we derive some elliptic estimates for the linearization of the anti-self-dual equation in Section 5.2.

1.3. Outline of the proofs. Assertion (1) of Theorem 1.2 is proved in Section 3. The proof that the projection map $\pi: \mathbf{B}_{A_{0}}^{4}\left(\varepsilon_{0}\right) \rightarrow \mathcal{B}_{E}^{k}$ is a local diffeomorphism away from connections with non-minimal stabilizer essentially follows Uhlenbeck's verification of "openness" in her proof of Theorem 2.1 in $[\mathbf{2 6}]$ via the method of continuity (see Lemma 3.6). The proof that the $L^{4}$ ball $\mathbf{B}_{A_{0}}^{4}\left(\varepsilon_{0}\right)$ injects into the quotient (see Lemma 3.7) was suggested to us by Mrowka. The remainder of our article is taken up with the proof of Theorem 1.1 and hence Assertion (2) of Theorem 1.2.

In Section 4 we introduce the family of critical-exponent Sobolev norms, $L_{k, A_{0}}^{\sharp, 2}, k=0,1,2$, used to complete the proof of Theorem 1.1 and in Section 5 we describe the crucial embedding theorems enjoyed by those Sobolev spaces, as well as estimates for the Green's operator of the Laplacian $d_{A_{0}}^{*} d_{A_{0}}$. In particular, $L_{k, A_{0}}^{p} \subset L_{k, A_{0}}^{\sharp, 2}$, for every $p>2$ while, in the other direction, $L_{2, A_{0}}^{\sharp, 2} \subset C^{0}$. The latter embedding is the key motivation for the definition of these norms and it greatly facilitates the derivation of Green's operator estimates, in a wide number of applications in gauge theory [23], [24], with minimal dependence on the curvature of the connection $A_{0}$. The main ideas and embedding results in Sections 4 and 5 are due to Taubes [21], [23], [24], [25], so these sections are essentially expository. An earlier exposition from a somewhat different perspective, due to Donaldson, of Taubes' methods and some applications appears in [4]. The estimates of Section 5 are stated only in the four-dimensional case. While we might expect all of them to hold, in some form, for higher dimensions we confine our attention to dimension four as our intended applications are primarily concerned with smooth fourmanifold topology. In essence, the critical-exponent norms make a virtue out of necessity of the familiar fact that while the Green's operator of the Laplacian $d^{*} d$ on $C^{\infty}(X)$ maps $L^{p}(X)$ into $L^{2 p /(2-p)}(X)$ for $1<p<2$, it does not map $L^{2}(X)$ into $L^{\infty}(X)$ [19, Chapter V]. We recall that an Orlicz space $L_{\varphi}$ can be used to provide the "best target space" for an embedding of $L_{2}^{2}(X)$ [1, Chapter 8]. Here, we may instead view $L_{2}^{\sharp, 2}(X)$ as providing 
the "best domain space" for an embedding into $L^{\infty}(X)$, since $L_{2}^{p}(X) \subset$ $L_{2}^{\sharp, 2}(X) \subset L^{\infty}(X)$ for all $p>2$.

We give two proofs of Theorem 1.1. For our first proof, in Section 6, we essentially follow the strategy of Uhlenbeck [26] and apply the method of continuity. The difficult step here (in establishing openness - see Section 6.3) is to prove that the intrinsic, gauge-invariant $\mathcal{L}_{1, A_{0}}^{\sharp, 2}$ and $L_{1, A_{0}}^{\sharp, 2}$ distances in the quotient $\mathcal{B}_{E}^{k}$ bound the $L^{2 \sharp, 4}$ and $L_{1, A_{0}}^{2}$ norms in the slice $\mathbf{S}_{A_{0}} \subset \mathcal{A}_{E}^{k}$ : This is the point in our first proof where we use the critical-exponent estimates derived in Section 5 to control gauge transformations. The proof of "closedness" uses a compactness argument and is given in Section 6.2. The proofs of Theorems 1.1 and 1.2 are completed in Section 6.3.

Our second proof of Theorem 1.1 occupies Sections 7 and 8. In Section 7 we show that the exponential map Exp : $\Omega^{0}\left(\mathfrak{g}_{E}\right) \rightarrow \mathcal{G}_{E}$ extends to a continuous map Exp : $L_{2}^{\sharp, 2}\left(\mathfrak{g}_{E}\right) \rightarrow L_{2}^{\sharp, 2}\left(\mathfrak{g}_{E}\right)$ and that the resulting space of $L_{2}^{\sharp, 2}$-gauge transformations $\mathcal{G}_{E}^{2, \sharp, 2}$ is a Banach Lie group. In particular, $L_{2}^{\sharp, 2}$-gauge transformations are continuous and are contained in $\mathcal{G}_{E}^{2, p}$ for every $p>2$. The Sobolev multiplication and composition results for the critical-exponent norms then allow us to apply the inverse function theorem directly in Section 8, while still ensuring that all constants depend at most on $\nu_{0}\left[A_{0}\right]$ and $\left\|F_{A_{0}}\right\|_{L^{2}}$. We first use the compactness result of Section 6.1 to establish the existence of gauge transformations $w$ in $\mathcal{G}_{E}^{3}$ which minimize the $\mathcal{L}_{1, A_{0}}^{\sharp, 2}$ and $L_{1, A_{0}}^{\sharp, 2}$ distances in the quotient $\mathcal{B}_{E}^{k}$. Then, assuming the norm $\left\|w(A)-A_{0}\right\|_{L^{2 \sharp, 4}}$ or $\left\|w(A)-A_{0}\right\|_{L_{1, A_{0}}^{2}}$ is sufficiently small, we use the Sobolev embedding and multiplication theorems of Sections 4, 5, and 7 and a quantitative version of the inverse function theorem to prove the existence of a gauge transformation $v \in \mathcal{G}_{E}^{3}$ such that $d_{A_{0}}^{*}\left(u(A)-A_{0}\right)=0$, $u=v w \in \mathcal{G}_{E}^{k+1}$, and $\left\|u(A)-A_{0}\right\|_{L^{2 \sharp, 4}}$ and $\left\|u(A)-A_{0}\right\|_{L_{1, A_{0}}^{2}}$ are controlled by $\operatorname{dist}_{\mathcal{L}_{1, A_{0}}^{\sharp, 2}}\left([A],\left[A_{0}\right]\right)$ and $\operatorname{dist}_{L_{1, A_{0}}^{\sharp, 2}}\left([A],\left[A_{0}\right]\right)$, respectively.

Acknowledgments. We warmly thank Tom Mrowka for helpful and stimulating discussions on gauge theory and his suggestion that $L^{4}$-balls inject into the quotient, and warmly thank Cliff Taubes for very helpful discussions and especially for explaining his ideas and methods in [21], [23]. We would also like to thank the Harvard Mathematics Department, where this article was completed in September 1996, and the National Science Foundation for their generous hospitality and support during the preparation of this article.

\section{Preliminaries.}

We assume throughout this article that $X$ is a closed, connected, smooth, four-manifold with Riemannian metric $g$. Let $G$ be a compact Lie group with matrix representation $\rho: G \subset \mathrm{SO}(\mathbb{E})=\mathrm{SO}(r)$ where $\mathbb{E} \simeq \mathbb{R}^{r}$ as a real 
inner product space, let $P$ be a principal $G$ bundle, and let $E=P \times_{\rho} \mathbb{E}$ be the corresponding Riemannian vector bundle associated to $P$ by the representation $\rho$. Let $\mathfrak{g}_{E} \subset \mathfrak{g l}(E)$ be the bundle of Lie algebras associated to $P$ via the adjoint representation $\mathrm{Ad}: G \rightarrow \operatorname{Aut}(\mathfrak{g})$ of $G$ on its Lie algebra $\mathfrak{g}$ and viewed as a subbundle of $\mathfrak{g l}(E)$ via the induced representation $\rho_{*}: \mathfrak{g} \subset \mathfrak{s o}(\mathbb{E})$.

Given the covariant derivative $\nabla_{A}: C^{\infty}(E) \rightarrow C^{\infty}\left(T^{*} X \otimes E\right)$, we define the exterior covariant derivative $d_{A}: \Omega^{i}(E) \rightarrow \Omega^{i+1}(E)$ in the usual way by setting $d_{A}=\nabla_{A}$ on $\Omega^{0}(E)=C^{\infty}(E)$ and extending $d_{A}$ to $\Omega^{i}(E)=$ $C^{\infty}\left(\Lambda^{i} \otimes E\right)$, where $\Lambda^{i}:=\Lambda^{i}\left(T^{*} X\right)$, according to the rule $d_{A}(\omega \wedge v)=$ $d \omega \wedge v+(-1)^{i} \omega \wedge d_{A} v$ for $\omega \in \Omega^{i}(X)$ and $v \in \Omega^{j}(E)$.

For any integer $k \geq 0$, exponent $1 \leq p \leq \infty$, and $L_{k}^{p}$ connection $A_{0}$ on $E$ we define the $L_{k}^{p}$ Sobolev completion, $L_{k}^{p}\left(\Lambda^{\ell} \otimes E\right)$, of $\Omega^{\ell}(E)$ with respect to the norm

$$
\|s\|_{L_{k, A_{0}}^{p}(X)}:=\left(\sum_{j=0}^{k}\left\|\nabla_{A_{0}}^{j} s\right\|_{L^{p}(X)}^{p}\right)^{1 / p} .
$$

We define the action of a $C^{\infty}$ gauge transformation $u \in \mathcal{G}_{E}$ on a $C^{\infty}$ connection $A$ on the bundle $E$ by pushforward, so $u(A):=A-\left(d_{A} u\right) u^{-1}$. Fix a connection $A_{0} \in \mathcal{A}_{E}$, let $\mathcal{A}_{E}^{k}=A_{0}+L_{k}^{2}\left(\Lambda^{1} \otimes \mathfrak{g}_{E}\right)$, and define

$$
\mathcal{G}_{E}^{k+1}:=\left\{u \in L_{k+1}^{2}(\mathfrak{g l}(E)): u \in G \text { a.e. }\right\} \subset L_{k+1}^{2}(\mathfrak{g l}(E)) .
$$

The space $\mathcal{G}_{E}^{k+1}$ is a Banach Lie group, with Lie algebra $T_{\mathrm{id}_{E}} \mathcal{G}_{E}^{k+1}=L_{k+1}^{2}\left(\mathfrak{g}_{E}\right)$, and acts smoothly on $\mathcal{A}_{E}^{k}$ with quotient $\mathcal{B}_{E}^{k}:=\mathcal{A}_{E}^{k} / \mathcal{G}_{E}^{k+1}$ endowed with the quotient $L_{k}^{2}$ topology.

The stabilizer subgroup $\operatorname{Stab}_{A} \subset \mathcal{G}_{E}^{k+1}$ for a connection $A$ on $E$ always contains the center $\operatorname{Center}(G) \subset G$. We let $\mathcal{A}_{E}^{*, k} \subset \mathcal{A}_{E}^{k}$ denote the space of connections $A \in \mathcal{A}_{E}^{k}$ with minimal stabilizer $\operatorname{Stab}_{A}=\operatorname{Center}(G)$ and let $\mathcal{B}_{E}^{*, k}=\mathcal{A}_{E}^{*, k} / \mathcal{G}_{E}^{k+1}$. As usual, the stabilizer subgroup $\operatorname{Stab}_{A} \subset \mathcal{G}_{E}$ can be identified with a closed subgroup of $G \subset \mathrm{GL}\left(\left.E\right|_{x_{0}}\right)$ for any point $x_{0} \in X$ by parallel translation with respect to the connection $A$. Let $\mathfrak{s t a \mathfrak { b } _ { A }}$ denote the Lie algebra of $\operatorname{Stab}_{A}$, so $\mathfrak{s t a b}_{A}=\operatorname{Ker}\left\{d_{A}: L_{k+1}^{2}\left(\mathfrak{g}_{E}\right) \rightarrow L_{k}^{2}\left(\Lambda^{1} \otimes \mathfrak{g}_{E}\right)\right\}$.

Throughout the article, we use $c$ or $z$ to denote positive constants which depend at most on the Riemannian manifold $(X, g)$ and the group $G$; constants may increase from one line to the next and are not renamed unless clarity demands otherwise.

\section{The slice theorem.}

In this section we prove the first assertion of Theorem 1.2 - see Proposition 3.4 below - namely, that a small enough $L^{4}$-ball $\mathbf{B}_{A_{0}}^{4}\left(\varepsilon_{0}\right) / \mathrm{Stab}_{A_{0}}$ provides a slice for the action of $\mathcal{G}_{E}^{k+1}$. The proof that the projection $\pi$ : 
$\mathbf{B}_{A_{0}}^{4}\left(\varepsilon_{0}\right) / \operatorname{Stab}_{A_{0}} \rightarrow \mathcal{B}_{E}^{k}$ is injective (Lemma 3.7) was suggested to us by Mrowka.

Let $k \geq 2$ be an integer. The Banach Lie group $\mathcal{G}_{E}^{k+1}$ has Lie algebra $T_{\mathrm{id}_{E}} \mathcal{G}_{E}^{k+1}=L_{k+1}^{2}\left(\mathfrak{g}_{E}\right)$ and exponential map Exp : $L_{k+1}^{2}\left(\mathfrak{g}_{E}\right) \rightarrow \mathcal{G}_{E}^{k+1}$ given by $\zeta \mapsto u=\operatorname{Exp} \zeta$. Recall that $\operatorname{Stab}_{A}=\left\{\gamma \in \mathcal{G}_{E}^{k+1}: \gamma(A)=A\right\}$ may be identified with a Lie subgroup of $G$ and has Lie algebra $\mathfrak{s t a b}_{A}=$ $\operatorname{Ker}\left(\left.d_{A}\right|_{L_{k+1}^{2}}\right)$. The operator $d_{A}^{*}: L_{k+2}^{2}\left(\Lambda^{1} \otimes \mathfrak{g}_{E}\right) \rightarrow L_{k+1}^{2}\left(\mathfrak{g}_{E}\right)$ has closed range and we have an $L^{2}$-orthogonal decomposition

$$
\begin{aligned}
T_{\mathrm{id}_{E}} \mathcal{G}_{E}^{k+1} & =L_{k+1}^{2}\left(\mathfrak{g}_{E}\right) \\
& =\left(\operatorname{Ker}\left(\left.d_{A}\right|_{L_{k+1}^{2}}\right)\right)^{\perp} \oplus \operatorname{Ker}\left(\left.d_{A}\right|_{L_{k+1}^{2}}\right) \\
& =\operatorname{Im}\left(\left.d_{A}^{*}\right|_{L_{k+2}^{2}}\right) \oplus \operatorname{Ker}\left(\left.d_{A}\right|_{L_{k+1}^{2}}\right) \\
& =\left(\operatorname{Ker}\left(\left.d_{A}\right|_{L_{k+1}^{2}}\right)\right)^{\perp} \oplus \mathfrak{s t a \mathfrak { b } _ { A } .}
\end{aligned}
$$

Let $\operatorname{Stab}_{A}^{\perp}=L_{k+1}^{2} \cap \operatorname{Stab}_{A}^{\perp}=\operatorname{Exp}\left(\left(\left.\operatorname{Ker} d_{A}\right|_{L_{k+1}^{2}}\right)^{\perp}\right)$, the second equality following from the Sobolev composition lemma. The subspace $\operatorname{Stab}_{A}^{\perp} \subset \mathcal{G}_{E}^{k+1}$ is closed and is a Banach submanifold of $\mathcal{G}_{E}^{k+1}$ with codimension $\operatorname{dim} \mathfrak{s t a b} \mathfrak{b}_{A}$. From Claim 3.5 below we see that $\operatorname{Stab}_{A}^{\perp}$ is a slice near $\operatorname{id}_{E} \in \mathcal{G}_{E}^{k+1}$ for the right action of $\operatorname{Stab}_{A}$ on $\mathcal{G}_{E}^{k+1}$.

The map $d_{A}: L_{k+1}^{2}\left(\mathfrak{g}_{E}\right) \rightarrow L_{k}^{2}\left(\Lambda^{1} \otimes \mathfrak{g}_{E}\right)$ has closed range and so we have an $L^{2}$-orthogonal decomposition

$$
\begin{aligned}
T_{A} \mathcal{A}_{E}^{k} & =L_{k}^{2}\left(\Lambda^{1} \otimes \mathfrak{g}_{E}\right) \\
& =\operatorname{Im}\left(\left.d_{A}\right|_{L_{k+1}^{2}}\right) \oplus \operatorname{Ker}\left(\left.d_{A}^{*}\right|_{L_{k}^{2}}\right) \\
& =\operatorname{Im}\left(\left.d_{A}\right|_{L_{k+1}^{2}}\right) \oplus \mathbf{K}_{A},
\end{aligned}
$$

of the tangent space to the space of $L_{k}^{2}$ connections at $A$, where $\mathbf{K}_{A}=$ $\operatorname{Ker}\left(\left.d_{A}^{*}\right|_{L_{k}^{2}}\right)$.

The slice $\mathbf{S}_{A} \subset \mathcal{A}_{E}^{k}$ through a connection $A$ is given by $\mathbf{S}_{A}=A+\mathbf{K}_{A}$. If $\pi$ is the projection from $\mathcal{A}_{E}^{k}$ onto $\mathcal{B}_{E}^{k}=\mathcal{A}_{E}^{k} / \mathcal{G}_{E}^{k+1}$, denoted by $A \mapsto[A]$, we let

$$
\begin{aligned}
\mathbf{B}_{A}(\varepsilon) & =\left\{A_{1} \in \mathbf{S}_{A}:\left\|A_{1}-A\right\|_{L_{k ; A}^{2}}<\varepsilon\right\} \\
& =A+\left\{a \in \mathbf{K}_{A}:\|a\|_{L_{k ; A}^{2}}<\varepsilon\right\}
\end{aligned}
$$

be the open $L_{k}^{2}$-ball in $\mathbf{S}_{A}$ with center $A$ and $L_{k, A^{2}}^{2}$ radius $\varepsilon$. Similarly, we let

$$
\begin{aligned}
\mathbf{B}_{A}^{4}(\varepsilon) & =\left\{A_{1} \in \mathbf{S}_{A}:\left\|A_{1}-A\right\|_{L^{4}}<\varepsilon\right\} \\
& =A+\left\{a \in \mathbf{K}_{A}:\|a\|_{L^{4}}<\varepsilon\right\}
\end{aligned}
$$


be the open ball in $\mathbf{S}_{A}$ with center $A$ and $L^{4}$-radius $\varepsilon$.

The proof that the quotient space $\mathcal{A}_{E}^{k}$ is Hausdorff makes use of the following well-known technical result [10, Proposition A.5]. Note that the space $\mathcal{G}_{E}^{2}$ is neither a Banach Lie group nor does it act smoothly on $\mathcal{A}_{E}^{k}$ for $k \geq 1$.

Lemma 3.1. Let $E$ be a Hermitian bundle over a Riemannian manifold $X$ and let $k \geq 2$ be an integer. Suppose $\left\{A_{\alpha}\right\}$ and $\left\{B_{\alpha}\right\}$ are sequences of $L_{k}^{2}$ unitary connections on $E$ and that $\left\{u_{\alpha}\right\}$ is a sequence in $\mathcal{G}_{E}^{2}$ such that $u_{\alpha}\left(A_{\alpha}\right)=B_{\alpha}$. Then the following hold.

(1) The sequence $\left\{u_{\alpha}\right\}$ is in $\mathcal{G}_{E}^{k+1}$;

(2) If $\left\{A_{\alpha}\right\}$ and $\left\{B_{\alpha}\right\}$ converge in $\mathcal{A}_{E}^{k}$ to limits $A_{\infty}, B_{\infty}$, then there is a subsequence $\left\{\alpha^{\prime}\right\} \subset\{\alpha\}$ such that $\left\{u_{\alpha^{\prime}}\right\}$ converges in $\mathcal{G}_{E}^{k+1}$ to $u_{\infty}$ and $B_{\infty}=u_{\infty}\left(A_{\infty}\right)$.

We shall need the following quantitative version of the inverse function theorem here and especially in Section 8:

Theorem 3.2. Let $\mathbf{\Phi}: \mathbf{E} \rightarrow \mathbf{F}$ be a $C^{\ell}$ map of Banach spaces, for some $\ell \geq 1$, such that the differential $(D \mathbf{\Phi})_{x_{0}}: \mathbf{E} \rightarrow \mathbf{F}$ has a continuous inverse $(D \Phi)_{x_{0}}^{-1}: \mathbf{F} \rightarrow \mathbf{E}$ satisfying

$$
\left\|(D \boldsymbol{\Phi})_{x_{0}}^{-1}\right\| \leq K \quad \text { and } \quad\left\|(D \boldsymbol{\Phi})_{x}-(D \boldsymbol{\Phi})_{x_{0}}\right\| \leq \frac{1}{2} K^{-1}, \quad \text { if } \quad\left\|x-x_{0}\right\| \leq \delta,
$$

for some positive constants $K$ and $\delta$. Then the following hold:

(1) The restriction of $\boldsymbol{\Phi}$ to the ball $U=B^{\mathbf{E}}\left(x_{0}, \delta\right)$ is injective and $\mathbf{\Phi}(U)=$ $V$ is an open set in $\mathbf{F}$ containing the ball $B^{\mathbf{F}}\left(\mathbf{\Phi}\left(x_{0}\right), \delta /(2 K)\right)$;

(2) The inverse map $\boldsymbol{\Phi}^{-1}: V \rightarrow U$ is $C^{\ell}$;

(3) If $x_{1}, x_{2} \in B^{\mathbf{E}}\left(x_{0}, \delta\right)$, then $\left\|x_{1}-x_{2}\right\| \leq 2 K\left\|\mathbf{\Phi}\left(x_{1}\right)-\mathbf{\Phi}\left(x_{2}\right)\right\|$.

For quantitative comparisons in this section, the following elementary fact will suffice:

Lemma 3.3. Let $\mathbf{E}, \mathbf{F}$ be Banach spaces and let $T \in \operatorname{Hom}(\mathbf{E}, \mathbf{F})$ have a right (left) inverse $S$. If $\tilde{T} \in \operatorname{Hom}(\mathbf{E}, \mathbf{F})$ satisfies $\|\tilde{T}-T\|<\|S\|^{-1}$, then $\tilde{T}$ also has a right (left) inverse.

Proof. If $S \in \operatorname{Hom}(\mathbf{F}, \mathbf{E})$ is a right inverse for $T$, so $T S=\mathrm{id}_{\mathbf{F}}$, then $\|(\tilde{T}-$ $T) S\|\leq\| \tilde{T}-T\|\| S \|<1$ and $\operatorname{id}_{\mathbf{E}}+(\tilde{T}-T) S$ is an invertible element of the Banach algebra End $(\mathbf{E})$. Define $\widetilde{S}=S(1+(\tilde{T}-T) S)^{-1}$, so $\widetilde{S} T=\operatorname{id}_{\mathbf{E}}$ and $\widetilde{S}$ is a right inverse for $\tilde{T}$. Similarly for left inverses.

This consequence of the usual characterization of invertible elements of a Banach algebra will be invoked in the proof of Lemma 3.6.

Proposition 3.4. Let $X$ be a closed, Riemannian four-manifold. Then there is a positive constant $z$ with the following significance. Let $E$ be a 
$G$ bundle over $X$. Suppose that $k \geq 2$ is an integer. Given $\left[A_{0}\right]$ in $\mathcal{B}_{E}^{k}$, let $\nu_{0}\left[A_{0}\right]$ be the least positive eigenvalue of the Laplacian $\Delta_{A_{0}}^{0}$ and let $\varepsilon_{0}$ be a constant satisfying $0<\varepsilon_{0}<z\left(1+\nu_{0}\left[A_{0}\right]^{-1 / 2}\right)^{-1}$. Then:

(1) The space $\mathcal{B}_{E}^{k}$ is Hausdorff;

(2) The subspace $\mathcal{B}_{E}^{*, k} \subset \mathcal{B}_{E}^{k}$ is open and is a $C^{\infty}$ Banach manifold with local parameterizations given by $\pi: \mathbf{B}_{A_{0}}^{4}\left(\varepsilon_{0}\right) \rightarrow \mathcal{B}_{E}^{*, k}$;

(3) The projection $\pi: \mathcal{A}_{E}^{*, k} \rightarrow \mathcal{B}_{E}^{*, k}$ is a $C^{\infty}$ principal $\mathcal{G}_{E}^{k+1} / \operatorname{Center}(G)$ bundle;

(4) The projection $\pi: \mathbf{B}_{A_{0}}^{4}\left(\varepsilon_{0}\right) / \mathrm{Stab}_{A_{0}} \rightarrow \mathcal{B}_{E}^{k}$ is a homeomorphism onto an open neighborhood of $\left[A_{0}\right] \in \mathcal{B}_{E}^{k}$ and a diffeomorphism on the subset where $\operatorname{Stab}_{A_{0}} / \operatorname{Center}(G)$ acts freely.

Proof. The stabilizer $\operatorname{Stab}_{A_{0}}$ acts freely on $\mathcal{G}_{E}^{k+1}$ and thus on the Banach manifold $\mathcal{G}_{E}^{k+1} \times \mathbf{S}_{A_{0}}^{k}$ by $(u, A) \mapsto \gamma \cdot(u, A)=\left(u \gamma^{-1}, \gamma(A)\right)$ and so the quotient $\mathcal{G}_{E}^{k+1} \times \operatorname{Stab}_{A_{0}} \mathbf{S}_{A_{0}}$ is again a Banach manifold. We define a smooth map

$$
\boldsymbol{\Psi}: \mathcal{G}_{E}^{k+1} \times \operatorname{Stab}_{A_{0}} \mathbf{S}_{A_{0}} \rightarrow \mathcal{A}_{E}^{k}, \quad[u, A] \mapsto u(A) .
$$

Our main task is to show that the map $\boldsymbol{\Psi}$ is (i) a local diffeomorphism onto its image and (ii) injective upon restriction to a sufficiently small neighborhood $\mathcal{G}_{E}^{k+1} \times \operatorname{Stab}_{A_{0}} \mathbf{B}_{A_{0}}^{4}\left(\varepsilon_{0}\right)$. Given $\delta_{0}>0$, let $B_{\mathrm{id}_{E}}\left(\delta_{0}\right)$ be the ball $\left\{u \in \mathcal{G}_{E}^{k+1}:\left\|u-\operatorname{id}_{E}\right\|_{L_{k+1 ; A_{0}}^{2}}<\delta_{0}\right\}$ and let $B_{\operatorname{id}_{E}}^{\perp}\left(\delta_{0}\right)=B_{\operatorname{id}_{E}}\left(\delta_{0}\right) \cap \operatorname{Stab}_{A_{0}}^{\perp}$.

Claim 3.5. For small enough $\delta=\delta\left(A_{0}, k\right)$, the ball $B_{\operatorname{id}_{E}}(\delta)$ is diffeomorphic to an open neighborhood in $B_{\mathrm{id}_{E}}^{\perp}(\delta) \times \operatorname{Stab}_{A_{0}}$, with inverse map given by $\left(u_{0}, \gamma\right) \mapsto u=u_{0} \gamma$

Proof. The differential of the multiplication map

$$
\operatorname{Stab}_{A_{0}}^{\perp} \times \operatorname{Stab}_{A_{0}} \rightarrow \mathcal{G}_{E}^{k+1}, \quad\left(u_{0}, \gamma\right) \mapsto u_{0} \gamma,
$$

at $\left(\operatorname{id}_{E}, \mathrm{id}_{E}\right)$ is given by

$$
\operatorname{Ker}\left(\left.d_{A_{0}}\right|_{L_{k+1}^{2}}\right)^{\perp} \oplus \mathfrak{s t a \mathfrak { b } _ { A _ { 0 } }} \rightarrow L_{k+1}^{2}\left(\mathfrak{g}_{E}\right) \quad(\zeta, \chi) \mapsto u_{0} \zeta \gamma+u_{0} \gamma \chi
$$

and so is just the identity map with respect to the $L^{2}$-orthogonal decomposition (3.1) of the range. Hence, the Banach space implicit function theorem implies that there is a diffeomorphism from an open neighborhood of $\left(\mathrm{id}_{E}, \mathrm{id}_{E}\right)$ onto an open neighborhood of $\operatorname{id}_{E} \in \mathcal{G}_{E}^{k+1}$. For small enough $\delta$, we may suppose that if $u \in B_{\operatorname{id}_{E}}(\delta)$, then $u$ can be written uniquely as $u=u_{0} \gamma$ with $u_{0} \in B_{\operatorname{id}_{E}}^{\perp}(\delta)$ and $\gamma \in \operatorname{Stab}_{A_{0}}$.

Lemma 3.6. For any $0<\varepsilon_{0}<\frac{1}{2}\left(1+\nu_{0}\left[A_{0}\right]^{-1 / 2}\right)^{-1}$, the map $\boldsymbol{\Psi}$ is a local diffeomorphism from $\mathcal{G}_{E}^{k+1} \times_{\operatorname{Stab}_{A_{0}}} \mathbf{B}_{A_{0}}^{4}\left(\varepsilon_{0}\right)$ onto its image in $\mathcal{A}_{E}^{k}$. 
Proof. We first restrict the map $\boldsymbol{\Psi}$ to a neighborhood $B_{\mathrm{id}_{E}}\left(\delta_{0}\right) \times \operatorname{Stab}_{A_{0}} \mathbf{S}_{A_{0}}$, which is diffeomorphic to the neighborhood $B_{\mathrm{id}_{E}}^{\perp}(\delta) \times \mathbf{S}_{A_{0}}$ in $\operatorname{Stab}_{A_{0}} \times \mathbf{S}_{A_{0}}$ by Claim 3.5. The differential of the induced map

$$
\boldsymbol{\Psi}: \operatorname{Stab}_{A_{0}}^{\perp} \times \mathbf{S}_{A_{0}} \rightarrow \mathcal{A}_{E}^{k}, \quad(u, A) \mapsto u(A),
$$

at $\left(\operatorname{id}_{E}, A\right):=\left(\operatorname{id}_{E}, A_{0}+a_{0}\right)$ is given by

$$
\begin{aligned}
(D \Psi)_{\left(\mathrm{id}_{E}, A\right)} & : T_{\operatorname{id}_{E}} \operatorname{Stab}_{A_{0}}^{\perp} \oplus T_{A} \mathbf{S}_{A_{0}} \rightarrow T_{A} \mathcal{A}_{E}^{k}, \\
(\zeta, a) & \mapsto-d_{A} \zeta+a=-d_{A_{0}} \zeta-\left[a_{0}, \zeta\right]+a,
\end{aligned}
$$

where we recall that $T_{A} \mathbf{S}_{A_{0}}=\mathbf{K}_{A_{0}}=\operatorname{Ker}\left(\left.d_{A_{0}}^{*}\right|_{L_{k}^{2}}\right)$ and

$$
T_{\mathrm{id}_{E}} \operatorname{Stab}_{A_{0}}^{\perp}=\left(\operatorname{Ker}\left(\left.d_{A_{0}}\right|_{L_{k+1}^{2}}\right)\right)^{\perp}=\operatorname{Im}\left(\left.d_{A_{0}}^{*}\right|_{L_{k+2}^{2}}\right) .
$$

Using the $L^{2}$-orthogonal decomposition (3.2) of the range we see that the map

$$
-d_{A_{0}} \oplus \operatorname{id}_{E}:\left(\operatorname{Ker}\left(\left.d_{A_{0}}\right|_{L_{1}^{2}}\right)\right)^{\perp} \oplus \operatorname{Ker}\left(\left.d_{A_{0}}^{*}\right|_{L_{1}^{2}}\right) \rightarrow \operatorname{Im}\left(\left.d_{A_{0}}\right|_{L_{1}^{2}}\right) \oplus \operatorname{Ker}\left(\left.d_{A_{0}}^{*}\right|_{L_{1}^{2}}\right)
$$

given by $(\zeta, b) \mapsto-d_{A_{0}} \zeta+b$ is a Hilbert space isomorphism. More explicitly, the operator

$$
d_{A_{0}}:\left(\operatorname{Ker}\left(\left.d_{A_{0}}\right|_{L_{1}^{2}}\right)\right)^{\perp} \rightarrow \operatorname{Im}\left(\left.d_{A_{0}}\right|_{L_{1}^{2}}\right)=\left(\operatorname{Ker}\left(\left.d_{A_{0}}^{*}\right|_{L^{2}}\right)\right)^{\perp}
$$

has a two-sided inverse

$$
G_{A_{0}}^{0} d_{A_{0}}^{*}: \operatorname{Im}\left(\left.d_{A_{0}}\right|_{L_{1}^{2}}\right) \rightarrow\left(\operatorname{Ker}\left(\left.d_{A_{0}}\right|_{L_{1}^{2}}\right)\right)^{\perp}
$$

where $G_{A_{0}}^{0}$ is the Green's operator for the Laplacian $\Delta_{A_{0}}^{0}=d_{A_{0}}^{*} d_{A_{0}}$ : Indeed, $G_{A_{0}}^{0} d_{A_{0}}^{*} d_{A_{0}}=G_{A_{0}}^{0} \Delta_{A_{0}}^{0}$ is the $L^{2}$-orthogonal projection $\Pi_{A_{0}}^{0}$ from $L_{1}^{2}\left(\Lambda^{1} \otimes \mathfrak{g}_{E}\right)$ onto $\left(\operatorname{Ker}\left(d_{A_{0}} \mid L_{1}^{2}\right)\right)^{\perp}$ and $d_{A_{0}} G_{A_{0}}^{0} d_{A_{0}}^{*}$ is the $L^{2}$-orthogonal projection $\Pi_{A_{0}}^{1, \perp}=$ id $-\Pi_{A_{0}}^{1}$ from $L^{2}\left(\Lambda^{1} \otimes \mathfrak{g}_{E}\right)$ onto $\left(\operatorname{Ker}\left(\left.d_{A_{0}}^{*}\right|_{L^{2}}\right)\right)^{\perp}$, as

$$
d_{A_{0}}^{*}\left(\mathrm{id}-d_{A_{0}} G_{A_{0}}^{0} d_{A_{0}}^{*}\right)=0 .
$$

For $\zeta \in\left(\operatorname{Ker}\left(\left.d_{A_{0}}\right|_{L_{1}^{2}}\right)\right)^{\perp}$ and $b=d_{A_{0}} \zeta \in \operatorname{Im}\left(\left.d_{A_{0}}\right|_{L_{1}^{2}}\right)$, we have

$$
\begin{aligned}
\left\|G_{A_{0}}^{0} d_{A_{0}}^{*} b\right\|_{L_{1, A_{0}}^{2}} & =\left\|G_{A_{0}}^{0} \Delta_{A_{0}}^{0} \zeta\right\|_{L_{1, A_{0}}^{2}}=\left\|\Pi_{A_{0}}^{0} \zeta\right\|_{L_{1, A_{0}}^{2}}=\|\zeta\|_{L_{1, A_{0}}^{2}} \\
& \leq\left\|d_{A_{0}} \zeta\right\|_{L^{2}}+\|\zeta\|_{L^{2}} \leq\left(1+\nu_{0}^{-1 / 2}\right)\left\|d_{A_{0}} \zeta\right\|_{L^{2}} \\
& =\left(1+\nu_{0}^{-1 / 2}\right)\|b\|_{L^{2}}
\end{aligned}
$$

and so $G_{A_{0}}^{0} d_{A_{0}}^{*}$ has $\operatorname{Hom}\left(L^{2}, L_{1, A_{0}}^{2}\right)$ operator norm bound

$$
\left\|G_{A_{0}}^{0} d_{A_{0}}^{*}\right\| \leq 1+\nu_{0}^{-1 / 2} \text {. }
$$


The Sobolev embedding $L_{1}^{2} \subset L^{4}$ and Kato's inequality imply that

$$
\left\|d_{A} \zeta-d_{A_{0}} \zeta\right\|_{L^{2}} \leq\left\|\left[a_{0}, \zeta\right]\right\|_{L^{2}} \leq 2\left\|a_{0}\right\|_{L^{4}}\|\zeta\|_{L^{4}} \leq 2\left\|a_{0}\right\|_{L^{4}}\|\zeta\|_{L_{1, A_{0}}^{2}},
$$

and so $d_{A}-d_{A_{0}}$ has $\operatorname{Hom}\left(L_{1, A_{0}}^{2}, L^{2}\right)$ operator norm bound

$$
\left\|d_{A}-d_{A_{0}}\right\| \leq 2\left\|a_{0}\right\|_{L^{4}} .
$$

In particular, we see that $(D \boldsymbol{\Psi})_{\left(\mathrm{id}_{E}, A_{0}\right)}^{-1}=G_{A_{0}}^{0} d_{A_{0}}^{*} \oplus \mathrm{id}=G_{A_{0}}^{0} d_{A_{0}}^{*} \oplus G_{A_{0}}^{0} \Delta_{A_{0}}^{0}$ satisfies

$\left\|(D \boldsymbol{\Psi})_{\left(\mathrm{id}_{E}, A_{0}\right)}^{-1}\right\| \leq 1+\nu_{0}^{-1 / 2}$ and $\left\|(D \boldsymbol{\Psi})_{\left(\mathrm{id}_{E}, A\right)}-(D \boldsymbol{\Psi})_{\left(\mathrm{id}_{E}, A_{0}\right)}\right\| \leq 2\left\|a_{0}\right\|_{L^{4}}$.

Hence, Lemma 3.3 implies that if $\left\|a_{0}\right\|_{L^{4}}<\frac{1}{2}\left(1+\nu_{0}^{-1 / 2}\right)^{-1}$, then the operator

$$
(D \Psi)_{\left(\mathrm{id}_{E}, A\right)}:\left(\operatorname{Ker}\left(\left.d_{A_{0}}\right|_{L_{1}^{2}}\right)\right)^{\perp} \times \operatorname{Ker}\left(\left.d_{A_{0}}^{*}\right|_{L_{1}^{2}}\right) \rightarrow L^{2}\left(\Lambda^{1} \otimes \mathfrak{g}_{E}\right)
$$

is an isomorphism from $L_{1}^{2}$ to $L^{2}$ and restricts to a bounded linear map from $L_{k+1}^{2}$ to $L_{k}^{2}$. Provided $(D \Psi)_{\left(\mathrm{id}_{E}, A\right)}: L_{k+1}^{2} \rightarrow L_{k}^{2}$ is bijective, the open mapping theorem guarantees the existence of a bounded inverse $(D \Psi)_{\left(\mathrm{id}_{E}, A\right)}^{-1}$ : $L_{k}^{2} \rightarrow L_{k+1}^{2}$. If $(D \Psi)_{\left(\operatorname{id}_{E}, A\right)}(\zeta, a)=0$ for $(\zeta, a) \in L_{k+1}^{2}$, then $(\zeta, a)$ is zero in $L_{1}^{2}$ and thus zero in $L_{k+1}^{2}$, so $(D \Psi)_{\left(\mathrm{id}_{E}, A\right)}$ is injective. If $b \in L_{k}^{2}\left(\Lambda^{1} \otimes \mathfrak{g}_{E}\right)$, then $b=(D \boldsymbol{\Psi})_{\left(\operatorname{id}_{E}, A\right)}(\zeta, a)=-d_{A} \zeta+a$ for some $(\zeta, a) \in\left(\operatorname{Ker}\left(\left.d_{A_{0}}\right|_{L_{1}^{2}}\right)\right)^{\perp} \times$ $\operatorname{Ker}\left(\left.d_{A_{0}}^{*}\right|_{L_{1}^{2}}\right)$. As $d_{A_{0}}^{*} a=0$, we have

$$
d_{A_{0}}^{*} d_{A} \zeta=-d_{A_{0}}^{*} b \in L_{k-1}^{2}
$$

and $d_{A_{0}}^{*} d_{A}: L_{k+1}^{2} \rightarrow L_{k-1}^{2}$ is an elliptic operator with $L_{k-1}^{2}$ coefficients. Thus, $\zeta \in L_{k+1}^{2}$, so $a=b+d_{A} \zeta \in L_{k}^{2}$, and $(D \Psi)_{\left(\mathrm{id}_{E}, A\right)}$ is surjective.

Combining the above observations, we see that the operator

$$
(D \Psi)_{\left(\mathrm{id}_{E}, A\right)}:\left(\operatorname{Ker}\left(\left.d_{A_{0}}\right|_{L_{k+1}^{2}}\right)\right)^{\perp} \oplus \operatorname{Ker}\left(\left.d_{A_{0}}^{*}\right|_{L_{k}^{2}}\right) \rightarrow L_{k}^{2}\left(\Lambda^{1} \otimes \mathfrak{g}_{E}\right),
$$

is an isomorphism for all $A=A_{0}+a_{0}$ with $\left\|a_{0}\right\|_{L^{4}}<\varepsilon_{0}=\frac{1}{2}\left(1+\nu_{0}^{-1 / 2}\right)^{-1}$. So, by the Banach space implicit function theorem, there are positive constants $\varepsilon=\varepsilon(A, k)$ and $\delta=\delta(A, k)$ and an open neighborhood $U_{A} \subset \mathcal{A}_{E}^{k}$ such that the map

$$
\Psi: B_{\mathrm{id}_{E}}^{\perp}(\delta) \times \mathbf{B}_{A}(\varepsilon) \rightarrow U_{A}, \quad\left(u, A_{1}\right) \mapsto u\left(A_{1}\right),
$$

with $\mathbf{B}_{A}(\varepsilon) \subset \mathbf{B}_{A_{0}}^{4}\left(\varepsilon_{0}\right)$, gives a diffeomorphism from an open neighborhood of $\left(\operatorname{id}_{E}, A\right)$ onto an open neighborhood of $A$. In particular, we obtain a map $U_{A} \rightarrow \operatorname{Stab}_{A_{0}}^{\perp}$, given by $A_{1} \mapsto u=u_{A_{1}}$, such that

$$
\Psi^{-1}\left(A_{1}\right)=\left(u, u^{-1}\left(A_{1}\right)\right) \in B_{\mathrm{id}_{E}}^{\perp}(\delta) \times \mathbf{B}_{A}(\varepsilon) \subset \operatorname{Stab}_{A_{0}}^{\perp} \times \mathbf{B}_{A_{0}}^{4}\left(\varepsilon_{0}\right) .
$$


Hence, for any $A_{1} \in U_{A}$ there is a unique $u \in B_{\operatorname{id}_{E}}^{\perp}(\delta)$ such that $u^{-1}\left(A_{1}\right)-$ $A_{0} \in \mathbf{K}_{A_{0}}$ :

$$
d_{A_{0}}^{*}\left(u^{-1}\left(A_{1}\right)-A_{0}\right)=0 .
$$

The neighborhood $\mathbf{B}_{A_{0}}^{4}\left(\varepsilon_{0}\right)$ is Stab $A_{A_{0}}$-invariant: If $A \in \mathbf{B}_{A_{0}}(\varepsilon)$ and $\gamma \in$ $\operatorname{Stab}_{A_{0}}$, then

$$
\left\|\gamma(A)-A_{0}\right\|_{L^{4}}=\left\|A-\gamma^{-1}\left(A_{0}\right)\right\|_{L^{4}}=\left\|A-A_{0}\right\|_{L^{4}}<\varepsilon,
$$

and

$$
\begin{aligned}
d_{A_{0}}^{*}\left(\gamma(A)-A_{0}\right) & =\gamma\left(d_{\gamma^{-1}\left(A_{0}\right)}^{*}\left(A-\gamma^{-1}\left(A_{0}\right)\right)\right) \\
& =\gamma\left(d_{A_{0}}^{*}\left(A-A_{0}\right)\right)=0,
\end{aligned}
$$

so $\gamma(A) \in \mathbf{B}_{A_{0}}(\varepsilon)$.

The group $\mathcal{G}_{E}^{k+1}$ acts on $\mathcal{G}_{E}^{k+1} \times \mathbf{S}_{A_{0}}$ by $(u, A) \mapsto(v u, A)$, and so gives a diffeomorphism

$$
B_{\mathrm{id}_{E}}(\delta) \times \mathbf{B}_{A_{0}}^{4}\left(\varepsilon_{0}\right) \rightarrow B_{v}(\delta) \times \mathbf{B}_{A_{0}}^{4}\left(\varepsilon_{0}\right), \quad(u, A) \rightarrow(v u, A),
$$

and as this action commutes with the given action of $\mathrm{Stab}_{A_{0}}$, it descends to a diffeomorphism

$$
B_{\mathrm{id}_{E}}(\delta) \times \operatorname{Stab}_{A_{0}} \mathbf{B}_{A_{0}}^{4}\left(\varepsilon_{0}\right) \rightarrow B_{v}(\delta) \times \operatorname{Stab}_{A_{0}} \mathbf{B}_{A_{0}}^{4}\left(\varepsilon_{0}\right), \quad[u, A] \rightarrow[v u, A],
$$

for each $v \in \mathcal{G}_{E}^{k+1}$. Consequently, the $\mathcal{G}_{E}^{k+1}$-equivariant map

$$
\mathcal{G}_{E}^{k+1} \times{ }_{\operatorname{Stab}_{A_{0}}} \mathbf{B}_{A_{0}}^{4}\left(\varepsilon_{0}\right) \rightarrow \mathcal{A}_{E}^{k}
$$

is a local diffeomorphism onto its image, as desired.

Plainly, $[\gamma(A)]=[A]$ for each $\gamma \in \operatorname{Stab}_{A_{0}}$ and $A \in \mathbf{B}_{A_{0}}^{4}\left(\varepsilon_{0}\right)$ and hence, the projection $\pi: \mathbf{B}_{A_{0}}^{4}\left(\varepsilon_{0}\right) \rightarrow \mathcal{A}_{E}^{k}$ factors through $\mathbf{B}_{A_{0}}^{4}(\varepsilon) / \operatorname{Stab}_{A_{0}}$.

Lemma 3.7. There is a positive constant $z$ with the following significance. Let $\nu_{0}\left[A_{0}\right]$ be the least positive eigenvalue of the Laplacian $\Delta_{A_{0}}^{0}$. Then for any constant $\varepsilon_{0}$ satisfying $0<\varepsilon_{0}<z\left(1+\nu_{0}\left[A_{0}\right]^{-1 / 2}\right)^{-1}$, the projection map $\pi: \mathbf{B}_{A_{0}}^{4}\left(\varepsilon_{0}\right) / \operatorname{Stab}_{A_{0}} \rightarrow \mathcal{B}_{E}^{k}$ is injective.

Proof. Suppose $A_{i} \in \mathbf{B}_{A_{0}}^{4}\left(\varepsilon_{0}\right)$ for $i=1,2$ and that $\left[A_{1}\right]=\left[A_{2}\right] \in \mathcal{B}_{E}^{k}$, so $u\left(A_{1}\right)=A_{2}$ for some $u \in \mathcal{G}_{E}^{k+1}$. Since $u\left(A_{0}\right)=A_{0}-\left(d_{A_{0}} u\right) u^{-1}$, we see that $u \in \operatorname{Stab}_{A_{0}}$ if and only $d_{A_{0}} u=0$. Here, we view $u \in L_{k+1}^{2}(\mathfrak{g l}(E))$ via the isometric embedding $\mathcal{G}_{E}^{k+1} \subset L_{k+1}^{2}(\mathfrak{g l}(E))$ and write

$$
u=u_{0}-\gamma,
$$

where $u_{0} \in\left(\operatorname{Ker} d_{A_{0}}\right)^{\perp}$ and $\gamma \in \operatorname{Ker} d_{A_{0}}$. We claim that $u_{0}=0$, so $u=\gamma \in$ $\operatorname{Stab}_{A_{0}}$. 
Since $u\left(A_{1}\right):=A_{1}-\left(d_{A_{1}} u\right) u^{-1}=A_{2}$, we have $A_{2} u=A_{1} u-d_{A_{1}} u=$ $A_{1} u-d_{A_{0}} u-\left[A_{1}-A_{0}, u\right]$, and therefore

$$
d_{A_{0}} u_{0}=d_{A_{0}} u=u\left(A_{1}-A_{0}\right)-\left(A_{2}-A_{0}\right) u \text {. }
$$

Since $d_{A_{0}}^{*}\left(A_{i}-A_{0}\right)=0$ for $i=1,2$, we obtain

$$
\begin{aligned}
d_{A_{0}}^{*} d_{A_{0}} u_{0}=- & *\left(d_{A_{0}} u \wedge *\left(A_{1}-A_{0}\right)\right)+u d_{A_{0}}^{*}\left(A_{1}-A_{0}\right) \\
& -\left(d_{A_{0}}^{*}\left(A_{2}-A_{0}\right)\right) u+*\left(*\left(A_{2}-A_{0}\right) \wedge d_{A_{0}} u\right) \\
=- & *\left(d_{A_{0}} u_{0} \wedge *\left(A_{1}-A_{0}\right)\right)+*\left(*\left(A_{2}-A_{0}\right) \wedge d_{A_{0}} u_{0}\right) .
\end{aligned}
$$

Integrating by parts gives

$$
\left\|d_{A_{0}} u_{0}\right\|_{L^{2}}^{2}=\left(d_{A_{0}}^{*} d_{A_{0}} u_{0}, u_{0}\right)_{2} \leq\left\|d_{A_{0}}^{*} d_{A_{0}} u_{0}\right\|_{L^{4 / 3}}\left\|u_{0}\right\|_{L^{4}} .
$$

Kato's inequality and the embedding $L_{1}^{2} \subset L^{4}$ gives $\left\|u_{0}\right\|_{L^{4}} \leq c\left(\left\|d_{A_{0}} u_{0}\right\|_{L^{2}}+\right.$ $\left\|u_{0}\right\|_{L^{2}}$ ), so the eigenvalue estimate $\left\|u_{0}\right\|_{L^{2}} \leq \nu_{0}^{-1 / 2}\left\|d_{A_{0}} u_{0}\right\|_{L^{2}}$ gives $\left\|u_{0}\right\|_{L^{4}} \leq$ $c\left(1+\nu_{0}^{-1 / 2}\right)\left\|d_{A_{0}} u_{0}\right\|_{L^{2}}$ and thus

$$
\left\|d_{A_{0}} u_{0}\right\|_{L^{2}}^{2} \leq\left(1+\nu_{0}^{-1 / 2}\right)\left\|d_{A_{0}}^{*} d_{A_{0}} u_{0}\right\|_{L^{4 / 3}}\left\|d_{A_{0}} u_{0}\right\|_{L^{2}} .
$$

Therefore, if $d_{A_{0}} u_{0} \not \equiv 0$, the preceding expression for $d_{A_{0}}^{*} d_{A_{0}} u_{0}$ yields

$$
\begin{aligned}
\left\|d_{A_{0}} u_{0}\right\|_{L^{2}} & \leq c\left(1+\nu_{0}^{-1 / 2}\right)\left\|d_{A_{0}}^{*} d_{A_{0}} u_{0}\right\|_{L^{4 / 3}} \\
& \leq c\left(1+\nu_{0}^{-1 / 2}\right)\left\|d_{A_{0}} u_{0}\right\|_{L^{2}}\left(\left\|A_{1}-A_{0}\right\|_{L^{4}}+\left\|A_{2}-A_{0}\right\|_{L^{4}}\right),
\end{aligned}
$$

and so we have

$$
1 \leq c\left(1+\nu_{0}^{-1 / 2}\right)\left(\left\|A_{1}-A_{0}\right\|_{L^{4}}+\left\|A_{2}-A_{0}\right\|_{L^{4}}\right) \leq c\left(1+\nu_{0}^{-1 / 2}\right) \varepsilon_{0}
$$

which gives a contradiction for $\varepsilon_{0}<c^{-1}\left(1+\nu_{0}^{-1 / 2}\right)^{-1}$.

We now return to consider the local diffeomorphism $\boldsymbol{\Psi}$ of Lemma 3.6. Suppose $\boldsymbol{\Psi}\left[u_{1}, A_{1}\right]=\boldsymbol{\Psi}\left[u_{2}, A_{2}\right] \in \mathcal{A}_{E}^{k}$, where $\left[u_{1}, A_{1}\right],\left[u_{2}, A_{2}\right] \in \mathcal{G}_{E}^{k+1} \times \operatorname{Stab}_{A_{0}}$ $\mathbf{B}_{A_{0}}^{4}\left(\varepsilon_{0}\right)$, and so $u_{1}\left(A_{1}\right)=u_{2}\left(A_{2}\right) \in \mathcal{A}_{E}^{k}$ and hence $\left[A_{1}\right]=\left[A_{2}\right] \in \mathcal{B}_{E}^{k}$. Provided $\varepsilon_{0}$ also satisfies the constraints of Lemma 3.7 , we have $u_{2}^{-1} u_{1}=$ $\gamma \in \operatorname{Stab}_{A_{0}}$ and $\gamma\left(A_{1}\right)=A_{2}$. Hence $\left[u_{2}, A_{2}\right]=\left[u_{1} \gamma^{-1}, \gamma\left(A_{1}\right)\right]=\left[u_{1}, A_{1}\right]$, so $\boldsymbol{\Psi}$ is injective and therefore a diffeomorphism onto $\mathcal{A}_{E}^{k}$.

The map $\pi: \mathbf{B}_{A_{0}}^{4}\left(\varepsilon_{0}\right) / \operatorname{Stab}_{A_{0}} \rightarrow \mathcal{B}_{E}^{k}$ can be factored as the composition of the inclusion $A \mapsto\left(\mathrm{id}_{E}, A\right)$ of $\mathbf{B}_{A_{0}}^{4}\left(\varepsilon_{0}\right)$ into $\mathcal{G}_{E}^{k+1} \times \mathbf{B}_{A_{0}}^{4}\left(\varepsilon_{0}\right)$, the projection onto the $\operatorname{Stab}_{A_{0}}$-quotient $\mathcal{G}_{E}^{k+1} \times \operatorname{Stab}_{A_{0}} \mathbf{B}_{A_{0}}^{4}\left(\varepsilon_{0}\right)$, the diffeomorphism $\boldsymbol{\Psi}$ of $\mathcal{G}_{E}^{k+1} \times{ }_{\operatorname{Stab}_{A_{0}}} \mathbf{B}_{A_{0}}^{4}\left(\varepsilon_{0}\right)$ with $\mathcal{A}_{E}^{k}$ and the projection from $\mathcal{A}_{E}^{k}$ onto the $\mathcal{G}_{E}^{k+1}$ quotient $\mathcal{B}_{E}^{k}=\mathcal{A}_{E}^{k} / \mathcal{G}_{E}^{k+1}$. Hence, $\pi$ is a homeomorphism onto an open neighborhood of $\left[A_{0}\right]$ in $\mathcal{B}_{E}^{k}$ and a diffeomorphism on the open subset where $\operatorname{Stab}_{A_{0}} / \operatorname{Center}(G)$ acts freely. 
Claim 3.8. The quotient space $\mathcal{B}_{E}^{k}$ is Hausdorff.

Proof. Let $\Gamma$ be the subspace $\left\{\{A, u(A)\}: A \in \mathcal{A}_{E}^{k}\right.$ and $\left.u \in \mathcal{G}_{E}^{k+1}\right\}$ of $\mathcal{A}_{E}^{k} \times$ $\mathcal{A}_{E}^{k}$. If $\left\{\left(A_{\alpha}\right), u_{\alpha}\left(A_{\alpha}\right)\right\}$ is a sequence in $\Gamma$ which converges in $L_{k}^{2}$ to a point $\left\{A_{\infty}, B_{\infty}\right\}$, then Lemma 3.1 implies that there is a subsequence $\left\{\alpha^{\prime}\right\} \subset\{\alpha\}$ such that $\left\{u_{\alpha}\right\}$ converges in $L_{k+1}^{2}$ to $u_{\infty} \in \mathcal{G}_{E}^{k+1}$ and $u_{\infty}\left(A_{\infty}\right)=B_{\infty}$. Thus, $\Gamma$ is closed and $\mathcal{A}_{E}^{k} / \mathcal{G}_{E}^{k+1}$ is Hausdorff.

Claim 3.8 gives Assertion (1) of the proposition and Assertions (2), (3), and (4) now follow from the preceding arguments and Lemma 3.7. This completes the proof of the proposition.

\section{Critical-exponent Sobolev norms.}

We now describe the basic properties of the critical-exponent norms and corresponding Banach spaces introduced by Taubes in [21], [23], [24], [25]. In particular, we give the basic embedding, multiplication, and composition lemmas we need to complete the proof of our slice theorem. We shall make frequent use of the pointwise Kato inequality, $|d| v|| \leq\left|\nabla_{A} v\right|$ for $v \in \Omega^{0}(E)$, so that the norms of the embedding and multiplication maps depend at most on the Riemannian manifold $(X, g)$. Moreover, for simplicity, we confine our attention to the case of closed four-manifolds: There are obvious analogues of the Sobolev lemmas described here for any $n$-manifold, with $n>2$. Similarly, extensions are possible to the case of complete manifolds bounded geometry (bounded curvature and injectivity radius uniformly bounded from below) - see [1], [3] for further details for Sobolev embedding results in those situations and for the construction of Green kernels. We refer the reader to the monograph of R. Adams [1] for a comprehensive treatment of Sobolev spaces and to that of E. Stein [19] for a treatment based on potential functions.

Throughout this section, $A, B$ denote $C^{\infty}$ orthogonal connections on Riemannian vector bundles $E, F$ over $X$ with $C^{\infty}$ sections $u, v$, respectively. We first have the following analogues of the $L^{2}$ and $L^{4}$ norms,

$$
\begin{aligned}
\|u\|_{L^{\sharp}(X)} & =\sup _{x \in X}\left\|\operatorname{dist}^{-2}(x, \cdot)|u|\right\|_{L^{1}(X)}, \\
\|u\|_{L^{2 \sharp}(X)} & =\sup _{x \in X}\left\|\operatorname{dist}^{-1}(x, \cdot)|u|\right\|_{L^{2}(X)},
\end{aligned}
$$

where $\operatorname{dist}(x, y)$ denotes the geodesic distance between points $x$ and $y$ in $X$ defined by the metric $g$; these norms have the same behavior as the $L^{2}$ and $L^{4}$ norms with respect to constant rescalings of the metric $g$ - the $L^{\sharp}$ norm on two-forms and the $L^{2 \sharp}$ norm on one-forms are scale invariant. Indeed, one sees this by noting that if $g \mapsto \tilde{g}=\lambda^{-2} g$, then $\operatorname{dist}_{\tilde{g}}(x, y)=\lambda^{-1} \operatorname{dist}_{g}(x, y)$ and $d V_{\tilde{g}}=\lambda^{-4} d V_{g}$, while for any $a \in \Omega^{1}(E)$ and $v \in \Omega^{2}(E)$, we have $|a|_{\tilde{g}}=\lambda|a|_{g}$, and $|v|_{\tilde{g}}=\lambda^{2}|v|_{g}$. 
Next, we define analogues of the $L_{1}^{2}$ and $L_{2}^{2}$ norms

$$
\begin{aligned}
\|u\|_{L_{1, A}^{2}(X)} & =\left\|\nabla_{A} u\right\|_{L^{2}(X)}+\|u\|_{L^{2}(X)}, \\
\|u\|_{L_{2, A}^{2}(X)} & =\left\|\nabla_{A}^{2} u\right\|_{L^{2}(X)}+\left\|\nabla_{A} u\right\|_{L^{2}(X)}+\|u\|_{L^{2}(X)},
\end{aligned}
$$

and set

$$
\begin{aligned}
& \|u\|_{L_{1, A}^{\sharp}(X)}=\left\|\nabla_{A} u\right\|_{L^{\sharp}(X)}+\|u\|_{L^{2 \sharp}(X)}+\|u\|_{L^{\sharp}(X)}, \\
& \|u\|_{L_{2, A}^{\sharp}(X)}=\left\|\nabla_{A}^{*} \nabla_{A} u\right\|_{L^{\sharp}(X)}+\|u\|_{L^{\sharp}(X)},
\end{aligned}
$$

where $\nabla_{A}^{*}=-* \nabla_{A^{*}}: \Omega^{1}(E) \rightarrow \Omega^{0}(E)$ is the $L^{2}$-adjoint of the map $\nabla_{A}: \Omega^{0}(E) \rightarrow \Omega^{1}(E)$.

Finally, we define analogues of the $C^{0} \cap L_{2}^{2}$ norm

$$
\|u\|_{C^{0} \cap L_{2, A}^{2}(X)}=\|u\|_{C^{0}(X)}+\|u\|_{L_{2, A}^{2}(X)},
$$

and set

$$
\begin{aligned}
\|u\|_{L^{\sharp, 2}(X)} & =\|u\|_{L^{\sharp} \cap L^{2}(X)}=\|u\|_{L^{\sharp}(X)}+\|u\|_{L^{2}(X)}, \\
\|u\|_{L^{2 \sharp, 4}(X)} & =\|u\|_{L^{2 \sharp} \cap L^{4}(X)}=\|u\|_{L^{2 \sharp}(X)}+\|u\|_{L^{4}(X)}, \\
\|u\|_{L_{1, A}^{\sharp, 2}(X)} & =\|u\|_{L_{1, A}^{\sharp} \cap L_{1, A}^{2}(X)}=\|u\|_{L_{1, A}^{\sharp}(X)}+\|u\|_{L_{1, A}^{2}(X)}, \\
\|u\|_{L_{2, A}^{\sharp, 2}(X)} & =\|u\|_{L_{2, A}^{\sharp} \cap L_{2, A}^{2}(X)}=\|u\|_{L_{2, A}^{\sharp}(X)}+\|u\|_{L_{2, A}^{2}(X)} .
\end{aligned}
$$

It might have appeared, at first glance, a little more natural to continue the obvious pattern and instead define $\|u\|_{L_{2, A}^{\sharp}(X)}$ using $\left\|\nabla_{A}^{2} u\right\|_{L^{\sharp}(X)}$ : As we shall see below, though, the given definition is most useful in practice. For related reasons, if $u \in \Omega^{1}(E)=\Omega^{0}\left(\Lambda^{1} \otimes E\right)$, it is convenient to define the norm $\|u\|_{L_{1, A}^{\sharp}(X)}$ by

$$
\|u\|_{L_{1, A}^{\sharp}(X)}=\left\|\nabla_{A}^{*} u\right\|_{L^{\sharp}(X)}+\|u\|_{L^{2 \sharp}(X)}+\|u\|_{L^{\sharp}(X)} .
$$

Let $L^{\sharp}(X)$ be the Banach space completion of $C^{\infty}(X)$ with respect to the norm $\|\cdot\|_{L^{\sharp}}$ and similarly define the remaining Banach spaces above.

We have the following extensions of the standard Sobolev embedding theorem [10], [15]: Their proofs are given in the next section. See also [4], [17], $[\mathbf{2 1}],[\mathbf{2 3}, \S 6],[\mathbf{2 4}$, Eq. (3.4) \& §5], and [25, Lemma 4.7].

Lemma 4.1. The following are continuous embeddings:

(1) $L_{k}^{p}(E) \subset L_{k}^{\sharp}(E)$, for $k=0,1,2$ and all $p>2$;

(2) $L^{q}(E) \subset L^{2 \sharp}(E)$, for all $q>4$;

(3) $L_{1}^{2}(E) \subset L^{2 \sharp}(E)$.

In the reverse direction we have:

Lemma 4.2. The following are continuous embeddings:

(1) $L^{\sharp}(E) \subset L^{1}(E)$ and $L^{2 \sharp}(E) \subset L^{2}(E)$;

(2) $L_{2}^{\sharp}(E) \subset C^{0} \cap L_{1}^{2}(E)$. 
We next consider the extension of the standard Sobolev multiplication lemma [10], [15]. While there is no continuous multiplication map $L_{2}^{2} \times$ $L_{2}^{2} \rightarrow L_{2}^{2}$, it is worth observing that there is a continuous bilinear map $C^{0} \cap L_{2}^{2}(E) \times C^{0} \cap L_{2}^{2}(F) \rightarrow C^{0} \cap L_{2}^{2}(E \otimes F)$ given by $(u, v) \mapsto u \otimes v$. Note that for $u \in \Omega^{0}(E)$ and $v \in \Omega^{0}(F)$ we have

$$
\begin{aligned}
\nabla_{A \otimes B}^{2}(u \otimes v)= & \left(\nabla_{A}^{2} u\right) \otimes v+2 \nabla_{A} u \otimes \nabla_{B} v+u \otimes \nabla_{B}^{2} v \\
\nabla_{A \otimes B}^{*} \nabla_{A \otimes B}(u \otimes v)= & \left(\nabla_{A}^{*} \nabla_{A} u\right) \otimes v+*\left(\left(* \nabla_{A} u\right) \wedge \nabla_{B} v\right) \\
& -*\left(\nabla_{A} u \wedge * \nabla_{B} v\right)+u \otimes \nabla_{B}^{*} \nabla_{B} v .
\end{aligned}
$$

Similarly, for $u \in \Omega^{0}\left(\Lambda^{1} \otimes E\right)$ and $v \in \Omega^{0}(F)$, we have

$$
\nabla_{A \otimes B}^{*}(u \otimes v)=\left(\nabla_{A}^{*} u\right) \otimes v+*\left(* u \wedge \nabla_{B} v\right) .
$$

In particular, we see that if $u, v \in \Omega^{0}(\mathfrak{g l}(E))$, then

$$
\begin{aligned}
\nabla_{A}^{*} \nabla_{A}(u v)= & \left(\nabla_{A}^{*} \nabla_{A} u\right) v+*\left(\left(* \nabla_{A} u\right) \wedge \nabla_{A} v\right) \\
& -*\left(\nabla_{A} u \wedge\left(* \nabla_{A} v\right)\right)+u\left(\nabla_{A}^{*} \nabla_{A} v\right),
\end{aligned}
$$

an identity we will need in the next section.

Lemma 4.3. Let $\Omega^{0}(E) \times \Omega^{0}(F) \rightarrow \Omega^{0}(E \otimes F)$ be given by $(u, v) \mapsto u \otimes v$. Then the following hold.

(1) The map $C^{0}(E) \otimes L^{\sharp}(F) \rightarrow L^{\sharp}(E \otimes F)$ is continuous;

(2) The map $L^{2 \sharp}(E) \otimes L^{2 \sharp}(F) \rightarrow L^{\sharp}(E \otimes F)$ is continuous;

(3) The spaces $L_{1}^{\sharp}(F), L_{1}^{2}(F)$, and $L_{2}^{\sharp}(F)$ are $L_{2}^{\sharp}(E)$-modules;

(4) The spaces $L_{1}^{2}(F), L_{1}^{\sharp, 2}(F)$, and $L_{2}^{\sharp, 2}(F)$ are $L_{2}^{\sharp, 2}(E)$-modules.

The conclusions continue to hold for $\Omega^{1}(E)$ in place of $\Omega^{0}(E)$ and the norms on $L_{1}^{\sharp}\left(\Lambda^{1} \otimes E\right)$ and $L_{1}^{\sharp, 2}\left(\Lambda^{1} \otimes E\right)$ defined via (4.4).

Proof. Let $u \in C^{\infty}(E)$ and $v \in C^{\infty}(F)$ and denote the covariant derivatives on $E, F$, and $E \otimes F$ by $\nabla$. Using $\nabla(u \otimes v)=(\nabla u) v+u \otimes \nabla v$ and the embedding $L_{2}^{\sharp}(E) \subset C^{0}(E)$, we see that

$$
\begin{aligned}
\|u \otimes v\|_{L^{\sharp}} & \leq\|u\|_{C^{0}}\|v\|_{L^{\sharp}} \quad \text { and } \quad\|u \otimes v\|_{L^{\sharp}} \leq\|u\|_{L^{2 \sharp}}\|v\|_{L^{2 \sharp}}, \\
\|\nabla(u \otimes v)\|_{L^{\sharp}} & \leq\|\nabla u\|_{L^{2 \sharp}}\|v\|_{L^{2 \sharp}}+\|u\|_{C^{0}}\|\nabla v\|_{L^{\sharp}} \leq c\|u\|_{L_{2}^{\sharp}}\|v\|_{L_{1}^{\sharp}}, \\
\|\nabla(u \otimes v)\|_{L^{2}} & \leq\|\nabla u\|_{L^{4}}\|v\|_{L^{4}}+\|u\|_{C^{0}}\|\nabla v\|_{L^{2}} \leq\|u\|_{C^{0} \cap L_{2}^{2}}\|v\|_{L_{1}^{2}},
\end{aligned}
$$

and hence the multiplication maps $C^{0} \times L^{\sharp} \rightarrow L^{\sharp}, L^{2 \sharp} \times L^{2 \sharp} \rightarrow L^{\sharp}$, and $L_{2}^{\sharp} \times L_{1}^{\sharp} \rightarrow L_{1}^{\sharp}$ are continuous. Moreover,

$$
\|\nabla(u \otimes v)\|_{L^{2}} \leq\|\nabla u\|_{L^{4}}\|v\|_{L^{4}}+\|u\|_{C^{0}}\|\nabla v\|_{L^{2}} \leq c\|u\|_{C^{0} \cap L_{2}^{2}}\|v\|_{L_{1}^{2}},
$$

and so, using the embedding $L_{2}^{\sharp} \subset C^{0}$, the multiplication $L_{2}^{\sharp, 2} \times L_{1}^{\sharp, 2} \rightarrow L_{1}^{\sharp, 2}$ is continuous. Thus, $L_{2}^{\sharp}$ is an $L_{1}^{\sharp}$-module and $L_{2}^{\sharp, 2}$ is an $L_{1}^{\sharp, 2}$-module. 
Finally, to see that $L_{2}^{\sharp}$ and $L_{2}^{\sharp, 2}$ are algebras, we use the identities (4.5), noting that

$$
\begin{aligned}
\left\|\nabla^{*} \nabla(u \otimes v)\right\|_{L^{\sharp}} & \leq\left\|\nabla^{*} \nabla u\right\|_{L^{\sharp}}\|v\|_{C^{0}}+2\|\nabla u\|_{L^{2 \sharp}}\|\nabla v\|_{L^{2 \sharp}}+\|u\|_{C^{0}}\left\|\nabla^{*} \nabla v\right\|_{L^{\sharp}} \\
& \leq c\|u\|_{L_{2}^{\sharp}}\|v\|_{L_{2}^{\sharp}},
\end{aligned}
$$

so the multiplication $L_{2}^{\sharp} \times L_{2}^{\sharp} \rightarrow L_{2}^{\sharp}$ is continuous, while

$$
\begin{aligned}
\left\|\nabla^{2}(u \otimes v)\right\|_{L^{2}} & \leq\left\|\nabla^{2} u\right\|_{L^{2}}\|v\|_{C^{0}}+2\|\nabla u\|_{L^{4}}\|\nabla v\|_{L^{4}}+\|u\|_{C^{0}}\left\|\nabla^{2} v\right\|_{L^{2}} \\
& \leq c\|u\|_{C^{0} \cap L_{2}^{2}}\|v\|_{C^{0} \cap L_{2}^{2}} .
\end{aligned}
$$

The embedding $L_{2}^{\sharp} \subset C^{0}$ now implies that the multiplication $L_{2}^{\sharp, 2} \times L_{2}^{\sharp 2} \rightarrow$ $L_{2}^{\sharp, 2}$ is continuous.

\section{Critical-exponent Sobolev embeddings and estimates for Green's operators.}

We continue the notation and assumptions of Section 4. Our goal in this section is to prove the Sobolev embedding Lemmas 4.1 and 4.2 , and to derive estimates for the Green's operator $G_{A}$ of the Laplacian $\nabla_{A}^{*} \nabla_{A}$ on $\Omega^{0}(E)$. The key estimates described in this section are due to Taubes and they arise, in a variety of contexts, in the proofs of [17, Lemma 5.4], [21, Equation (2.14) \& Lemmas 3.5, 3.6, \& A.3], [23, Equation (3.4b) \& Lemma 6.2], [24, Lemma 5.6], and $[\mathbf{2 5}, \S 4(\mathrm{c}),(\mathrm{d}),(\mathrm{e})]$. However, we find it convenient to collect them here - together with some useful extensions and generalizations - both for the purposes of the present article and applications in $[\mathbf{8}],[\mathbf{9}]$.

5.1. Estimates for the covariant Laplacian $\nabla_{A}^{*} \nabla_{A}$. Let $G(x, y)$ be the kernel function for the Green's operator $\left(d^{*} d+1\right)^{-1}$ of the Laplacian $d^{*} d+1$ on $C^{\infty}(X)$. The kernel $G(x, y)$ of $\left(d^{*} d+1\right)^{-1}$ behaves like $\operatorname{dist}^{-2}(x, y)$ as $\operatorname{dist}(x, y) \rightarrow 0$ (see [25, Lemma 4.7] and $[\mathbf{2 4}, \S 5]$ ):

Lemma 5.1. The kernel $G(x, y)$ is a positive $C^{\infty}$ function away from the diagonal in $X \times X$ and as $\operatorname{dist}(x, y) \rightarrow 0$,

$$
G(x, y)=\frac{1}{4 \pi^{2} \operatorname{dist}^{2}(x, y)}+o\left(\operatorname{dist}^{-2}(x, y)\right) .
$$

Proof. These and other properties of $G$ are obtained by explicitly constructing $G$ from an initial choice of parametrix $H$ for $d^{*} d+1$ using the method of $[3, \S 4.2 .2-3]$, where the kernel for the Green's operator for $d^{*} d$ is constructed. Recall from $\left[\mathbf{1 9}\right.$, p. 132] that the kernel $G_{0}(x, y)$ for $\left(d^{*} d+1\right)^{-1}$ on $\mathbb{R}^{4}$ with its standard metric satisfies

$$
G_{0}(x, y)=\frac{1}{4 \pi^{2}|x-y|^{2}}+o\left(|x-y|^{-2}\right), \quad|x-y| \rightarrow 0 .
$$

The kernel $G$ is now constructed using $G_{0}$ by following the method of [3, $\S 4.2 .2-3]$. 
Lemma 5.1 implies that there is a constant $c$ depending at most on $g$ such that for all $x \neq y$ in $X$,

$$
c^{-1} \operatorname{dist}^{-2}(x, y) \leq G(x, y) \leq c \operatorname{dist}^{-2}(x, y) .
$$

Consequently, for all $u \in \Omega^{0}(E)$, we have

$$
c^{-1}\|u\|_{L^{\sharp}(X)} \leq\|G|u|\|_{C^{0}(X)} \leq c\|u\|_{L^{\sharp}(X)} .
$$

Lemma 4.2 will follow from the next estimate; a similar inequality is stated as Equation (3.4) in [24]; see [17, Lemma 5.4(a)] for a related result on $\mathbb{R}^{3}$.

Lemma 5.2. For all $f \in L_{1}^{2}\left(\mathbb{R}^{4}\right)$, where $\mathbb{R}^{4}$ has its standard metric,

$$
\sup _{x \in \mathbb{R}^{4}}\left\|\operatorname{dist}^{-1}(x, \cdot) f\right\|_{L^{2}\left(\mathbb{R}^{4}\right)} \leq \frac{1}{2}\|\nabla f\|_{L^{2}\left(\mathbb{R}^{4}\right)} .
$$

Suppose $X$ be a closed, oriented, Riemannian four-manifold. Then there is a positive constant $c$ such that for all $f \in L_{1}^{2}(X)$,

$$
\sup _{x \in X}\left\|\operatorname{dist}^{-1}(x, \cdot) f\right\|_{L^{2}(X)} \leq c\|f\|_{L_{1}^{2}(X)} .
$$

Proof. Let $f \in C_{0}^{\infty}\left(\mathbb{R}^{4}\right)$ and let $x=(r, \theta)$ denote polar coordinates centered at a point $x_{0} \in \mathbb{R}^{4}$, so $r=\left|x-x_{0}\right|$. Then

$$
\begin{aligned}
\int_{\mathbb{R}^{4}} r^{-2}|f|^{2} d x & =\int_{S^{3}} \int_{\mathbb{R}} r f^{2} d r d \theta \\
& =\frac{1}{2} \int_{S^{3}} \int_{\mathbb{R}} \frac{d r^{2}}{d r} f^{2} d r d \theta=-\frac{1}{2} \int_{S^{3}} \int_{\mathbb{R}} r^{2} f \frac{\partial f}{\partial r} d r d \theta
\end{aligned}
$$

via integration by parts. Therefore,

$$
\begin{aligned}
\int_{\mathbb{R}^{4}} r^{-2}|f|^{2} d x & =-\frac{1}{2} \int_{\mathbb{R}^{4}} r^{-1} f \frac{\partial f}{\partial r} d x \\
& \leq \frac{1}{2}\left(\int_{\mathbb{R}^{4}} r^{-2}|f|^{2} d x\right)^{1 / 2}\left(\int_{\mathbb{R}^{4}}|\nabla f|^{2} d x\right)^{1 / 2} .
\end{aligned}
$$

Hence, for all $f \in C_{0}^{\infty}\left(\mathbb{R}^{4}\right)$ we have

$$
\left\|\operatorname{dist}^{-1}\left(x_{0}, \cdot\right) f\right\|_{L^{2}\left(\mathbb{R}^{4}\right)} \leq \frac{1}{2}\|\nabla f\|_{L^{2}\left(\mathbb{R}^{4}\right)},
$$

and taking the supremum over $x_{0} \in \mathbb{R}^{4}$ yields the first assertion.

For a closed Riemannian manifold $X$, choosing a smooth partition of unity for $X$ and applying first assertion (when $X$ is $\mathbb{R}^{4}$ ) to each patch then yields the second assertion.

Proof of Lemma 4.1. Define $1 \leq p^{\prime}<2$ by setting $1=1 / p+1 / p^{\prime}$. Then Hölder's inequality implies that

$$
\begin{aligned}
& \left\|\operatorname{dist}^{-2}(x, \cdot)|u|\right\|_{L^{1}} \leq\left\|\operatorname{dist}^{-2}(x, \cdot)\right\|_{L^{p^{\prime}}}\|u\|_{L^{p}} \leq C\|u\|_{L^{p}}, \\
& \left\|\operatorname{dist}^{-1}(x, \cdot)|u|\right\|_{L^{2}} \leq\left\|\operatorname{dist}^{-1}(x, \cdot)\right\|_{L^{2 p^{\prime}}}\|u\|_{L^{2 p}} \leq C\|u\|_{L^{2 p}},
\end{aligned}
$$


which gives Assertions (1) and (2). By Lemma 5.2 and Kato's inequality, $|d| u|| \leq\left|\nabla_{A} u\right|$, we see that

$$
\begin{aligned}
\sup _{x \in X}\left\|\operatorname{dist}^{-1}(x, \cdot) u\right\|_{L^{p}} & =\sup _{x \in X}\left\|\operatorname{dist}^{-1}(x, \cdot)|u|\right\|_{L^{p}} \\
& \leq C\left(\|d|u|\|_{L^{p}}+\|u\|_{L^{p}}\right) \\
& \leq C\left(\left\|\nabla_{A} u\right\|_{L^{p}}+\|u\|_{L^{p}}\right) .
\end{aligned}
$$

Taking $p=2$ gives Assertion (3).

Lemma 4.2 will follow from the estimates below; the key estimates (1) and (2) in Lemma 5.3 below and the estimates (1), (2), and (3) in Lemma 5.4 are essentially those of Lemma 6.2 in [23], except that the dependence of the constant on $\left\|F_{A}\right\|_{L^{2}}$ is made explicit, but the argument is the same as that of $[\mathbf{2 3}]$.

Lemma 5.3. Let $X$ be a closed, oriented four-manifold with metric g. Then there is a constant $c$ with the following significance. Let $E$ be a Riemannian vector bundle over $X$ and let $A$ be an orthogonal $L_{2}^{2}$ connection on $E$ with curvature $F_{A}$. Then $L_{2}^{\sharp}(E) \subset C^{0} \cap L_{1}^{2}(E)$ and the following estimates hold:

$$
\begin{aligned}
\left\|\nabla_{A} u\right\|_{L^{2 \sharp}(X)}+\|u\|_{C^{0}(X)} & \leq c\left\|\nabla_{A}^{*} \nabla_{A} u\right\|_{L^{\sharp}(X)}+\|u\|_{L^{\sharp}(X)}, \\
\left\|\nabla_{A} u\right\|_{L^{2 \sharp}(X)}+\|u\|_{C^{0}(X)} & \leq c\left\|\nabla_{A}^{*} \nabla_{A} u\right\|_{L^{\sharp}(X)}+\|u\|_{L^{2}(X)}, \\
\|u\|_{L^{1}(X)} & \leq c\|u\|_{L^{\sharp}(X)}, \\
\|u\|_{L^{2}(X)} & \leq c\|u\|_{L^{2 \sharp}(X)}, \\
\left\|\nabla_{A} u\right\|_{L^{2}(X)} & \leq c\left\|\nabla_{A} u\right\|_{L^{2 \sharp}(X)} .
\end{aligned}
$$

Proof. For any $u \in C^{\infty}(E)$ there is the following pointwise identity [10, p. 93],

$$
\left|\nabla_{A} u\right|^{2}+\frac{1}{2} d^{*} d|u|^{2}=\left\langle\nabla_{A}^{*} \nabla_{A} u, u\right\rangle
$$

and thus:

$$
\left|\nabla_{A} u\right|^{2}+\frac{1}{2}\left(1+d^{*} d\right)|u|^{2}=\left\langle\nabla_{A}^{*} \nabla_{A} u, u\right\rangle+\frac{1}{2}|u|^{2} .
$$

Using this identity and the fact that $\int_{X} G(x, \cdot)\left(d^{*} d+1\right)|u|^{2} d V=|u|^{2}(x)$, we obtain

$$
\begin{aligned}
& \int_{X} G(x, \cdot)\left|\nabla_{A} u\right|^{2} d V+\frac{1}{2}|u|^{2}(x) \\
& \leq \int_{X} G(x, \cdot)\left|\left\langle\nabla_{A}^{*} \nabla_{A} u, u\right\rangle\right| d V+\frac{1}{2} \int_{X} G(x, \cdot)|u|^{2} d V .
\end{aligned}
$$

Therefore, from (5.1), we have

$$
\begin{aligned}
\left\|\left|\nabla_{A} u\right|^{2}\right\|_{L^{\sharp}}+\left\||u|^{2}\right\|_{C^{0}} & \leq c\left\|\left\langle\nabla_{A}^{*} \nabla_{A} u, u\right\rangle\right\|_{L^{\sharp}}+c\left\|\left.|| u\right|^{2}\right\|_{L^{\sharp}} \\
& \leq c\left\|\nabla_{A}^{*} \nabla_{A^{u}} u\right\|_{L^{\sharp}}\|u\|_{C^{0}}+c\|u\|_{L^{\sharp}}\|u\|_{C^{0}} .
\end{aligned}
$$


Consequently, using rearrangement with the last term, we see that

$$
\left\|\nabla_{A} u\right\|_{L^{2 \sharp}}+\|u\|_{C^{0}} \leq c\left\|\nabla_{A}^{*} \nabla_{A} u\right\|_{L^{\sharp}}+c\|u\|_{L^{\sharp}},
$$

giving (1). Combining this estimate with the embedding and interpolation inequalities, $\|u\|_{L^{\sharp}} \leq c\|u\|_{L^{4}} \leq c\|u\|_{L^{2}}^{1 / 2}\|u\|_{C^{0}}^{1 / 2}$, and again using rearrangement with the last term yields the bound in (2). Since $X$ is closed, for all $x \neq y$ we have $\operatorname{dist}(x, y) \leq M<\infty$, so

$$
\int_{X} \operatorname{dist}^{-2}(x, \cdot)|u| d V \geq M^{-2} \int_{X}|u| d V,
$$

and this gives the estimates in (3), (4), and (5).

Proof of Lemma 4.2. From Lemma 5.3 we have the estimate

$$
\|u\|_{C^{0}} \leq c\|u\|_{L_{2, A_{0}}^{\sharp}},
$$

for any $u \in C^{\infty}(E)$. Let $\left\{u_{m}\right\}$ be a sequence in $C^{\infty}(E)$ converging to $u \in$ $L_{2}^{\sharp}(E)$. The sequence $\left\{u_{m}\right\}$ is Cauchy in $L_{2}^{\sharp}(E)$ and applying the preceding estimate to the differences $u_{m_{2}}-u_{m_{1}}$, we see that it is Cauchy in the Banach space $C^{0}(E)$ and so the limit $u$ lies in $C^{0}(E)$. The same argument, with estimates (1) and (5) of Lemma 5.3, shows that $u \in L_{1}^{2}(E)$ and this yields Assertion (2) of the lemma. Assertion (1) follows in the same manner.

Lemma 5.4. Continue the hypotheses of Lemma 5.3. Then for any $u \in$ $\left(C^{0} \cap L_{2}^{2}\right)(E)$, we have

$$
\begin{gathered}
\left\|\nabla_{A}^{2} u\right\|_{L^{2}(X)} \leq\left\|\nabla_{A}^{*} \nabla_{A} u\right\|_{L^{2}(X)}+c\left\|F_{A}\right\|_{L^{2}(X)}^{1 / 2}\left\|\nabla_{A} u\right\|_{L^{4}(X)} \\
+\left\|F_{A}\right\|_{L^{2}(X)}\|u\|_{C^{0}(X)},
\end{gathered}
$$

Proof. The Bochner-Weitzenböck formula for the covariant Laplacian [11, Appendix, Theorem II.1] asserts that

$$
d_{A}^{*} d_{A}+d_{A} d_{A}^{*}=\nabla_{A}^{*} \nabla_{A}+\left\{F_{A}, \cdot\right\}
$$

where we use $\{\cdot, \cdot\}$ to denote a certain bilinear map whose precise form is unimportant here. Integrating by parts and noting that $d_{A}=\nabla_{A}$ and $d_{A}^{*} d_{A}=\nabla_{A}^{*} \nabla_{A}$ on $\Omega^{0}(X, V)$ and $F_{A}=d_{A} \circ d_{A}$ gives

$$
\begin{aligned}
\left\|\nabla_{A}^{2} u\right\|_{L^{2}}^{2} & =\left(\nabla_{A}^{*} \nabla_{A} \nabla_{A} u, \nabla_{A} u\right)_{L^{2}} \\
& =\left(\left(d_{A}^{*} d_{A}+d_{A} d_{A}^{*}\right) d_{A} u, d_{A} u\right)_{L^{2}}-\left(\left\{F_{A}, d_{A} u\right\}, \nabla_{A} u\right)_{L^{2}} \\
& =\left(d_{A}^{*} F_{A} u, d_{A} u\right)_{L^{2}}+\left(d_{A}\left(d_{A}^{*} d_{A}\right) u, d_{A} u\right)_{L^{2}}-\left(\left\{F_{A}, \nabla_{A} u\right\}, \nabla_{A} u\right)_{L^{2}} \\
& =\left(F_{A} u, F_{A} u\right)_{L^{2}}+\left(\nabla_{A}^{*} \nabla_{A} u, \nabla_{A}^{*} \nabla_{A} u\right)_{L^{2}}-\left(\left\{F_{A}, \nabla_{A} u\right\}, \nabla_{A} u\right)_{L^{2}} .
\end{aligned}
$$


Therefore, applying Hölder's inequality, we find that

$$
\left\|\nabla_{A}^{2} u\right\|_{L^{2}}^{2} \leq\left\|\nabla_{A}^{*} \nabla_{A} u\right\|_{L^{2}}^{2}+c\left\|F_{A}\right\|_{L^{2}}\left\|\nabla_{A} u\right\|_{L^{4}}^{2}+\left\|F_{A}\right\|_{L^{2}}^{2}\|u\|_{C^{0}}^{2},
$$

and taking square roots gives the desired bound in (1).

We now use integration by parts and Kato's inequality $|d| u|| \leq\left|\nabla_{A} u\right|$ to obtain an $L^{4}$ bound on $d_{A} u$ :

$$
\begin{aligned}
\left\|d_{A} u\right\|_{L^{4}}^{4} & =\left(d_{A} u,\left|d_{A} u\right|^{2} d_{A} u\right)_{L^{2}} \\
& =\left(u,\left|d_{A} u\right|^{2} d_{A}^{*} d_{A} u\right)_{L^{2}}+2\left(u,\left|d_{A} u\right| d_{A} u \wedge d\left|d_{A} u\right|\right)_{L^{2}}, \\
& =\|u\|_{C^{0}}\left\|d_{A} u\right\|_{L^{4}}^{2}\left\|d_{A}^{*} d_{A} u\right\|_{L^{2}}+2\|u\|_{C^{0}}\left\|d_{A} u\right\|_{L^{4}}^{2}\left\|\nabla_{A} d_{A} u\right\|_{L^{2}},
\end{aligned}
$$

and so, if $d_{A} u \not \equiv 0$,

$$
\left\|d_{A} u\right\|_{L^{4}} \leq\|u\|_{C^{0}}^{1 / 2}\left(\left\|d_{A}^{*} d_{A} u\right\|_{L^{2}}+2\left\|\nabla_{A}^{2} u\right\|_{L^{2}}\right)^{1 / 2},
$$

which gives the desired estimate in (2).

By combining the $L^{4}$ estimate for $\nabla_{A} u$ with the $L^{2}$ estimate for $\nabla_{A}^{2} u$, we obtain

$$
\begin{aligned}
\left\|\nabla_{A}^{2} u\right\|_{L^{2}} \leq\left\|\nabla_{A}^{*} \nabla_{A} u\right\|_{L^{2}}+\left\|F_{A}\right\|_{L^{2}}\|u\|_{C^{0}} \\
\quad+c\left\|F_{A}\right\|_{L^{2}}^{1 / 2}\|u\|_{C^{0}}^{1 / 2}\left(\left\|\nabla_{A}^{*} \nabla_{A} u\right\|_{L^{2}}+\left\|\nabla_{A}^{2} u\right\|_{L^{2}}\right)^{1 / 2} .
\end{aligned}
$$

We now use rearrangement with the last term above to give

$$
\left\|\nabla_{A}^{2} u\right\|_{L^{2}} \leq 2\left\|\nabla_{A}^{*} \nabla_{A} u\right\|_{L^{2}}+c\left\|F_{A}\right\|_{L^{2}}\|u\|_{C^{0}},
$$

and this establishes the desired bound in (3).

Lemma 5.5. Continue the hypotheses of Lemma 5.3. Then for any $u \in$ $L_{2}^{\sharp, 2}(E)$, we have:

$$
\|u\|_{L_{2, A}^{2}(X)}+\|u\|_{C^{0}(X)} \leq c\left(1+\left\|F_{A}\right\|_{L^{2}(X)}\right)\left(\left\|\nabla_{A}^{*} \nabla_{A} u\right\|_{L^{\sharp, 2}(X)}+\|u\|_{L^{2}(X)}\right) .
$$

Proof. From Assertion (3) of Lemma 5.4 we have the estimate

$$
\left\|\nabla_{A}^{2} u\right\|_{L^{2}} \leq 2\left\|\nabla_{A}^{*} \nabla_{A} u\right\|_{L^{2}}+c\left\|F_{A}\right\|_{L^{2}}\|u\|_{C^{0}},
$$

while integration by parts gives

$$
\left\|\nabla_{A} u\right\|_{L^{2}}=\left(\nabla_{A}^{*} \nabla_{A} u, u\right)_{L^{2}}^{1 / 2} \leq \frac{1}{\sqrt{2}}\left(\left\|\nabla_{A}^{*} \nabla_{A} u\right\|_{L^{2}}+\|u\|_{L^{2}}\right) .
$$

According to Lemma 5.3 we have

$$
\|u\|_{C^{0}} \leq c\left\|\nabla_{A}^{*} \nabla_{A} u\right\|_{L^{\sharp}}+c\|u\|_{L^{2}},
$$

and therefore the desired bound follows by combining these estimates. 
The above lemmas lead to the following estimates for the Green's operator $G_{A}: L^{\sharp, 2}(E) \rightarrow L_{2}^{\sharp, 2}(E)$ of the Laplacian $\nabla_{A}^{*} \nabla_{A}: L_{2}^{\sharp, 2}(E) \rightarrow L^{\sharp, 2}(E)$. For $u \in \Omega^{0}(E)$ define

$$
\|u\|_{\mathcal{L}_{2, A}^{\sharp, 2}(X)}=\left\|\nabla_{A}^{*} \nabla_{A} u\right\|_{L^{\sharp, 2}(X)}+\|u\|_{L^{\sharp, 2}(X)},
$$

and observe that this is equivalent to the $L_{2, A}^{\sharp 2}$ norm defined in Section 4, although the comparison depends on the $L^{2}$ norm of the curvature $F_{A}$.

Lemma 5.6. Continue the hypotheses of Lemma 5.3. Let $\nu_{0}[A]$ be the least positive eigenvalue of the Laplacian $\nabla_{A}^{*} \nabla_{A}$. Then for any $u \in L^{\sharp, 2} \cap$ $\left(\operatorname{Ker} \nabla_{A}^{*} \nabla_{A}\right)^{\perp}$, we have:

$$
\begin{aligned}
\left\|G_{A} u\right\|_{L_{2, A}^{\sharp}(X)} & \leq c\left(1+\nu_{0}[A]^{-1}\right)\|u\|_{L^{\sharp, 2}(X)}, \\
\left\|G_{A} u\right\|_{\mathcal{L}_{2, A}^{\sharp, 2}(X)} & \leq c\left(1+\nu_{0}[A]^{-1}\right)\|u\|_{L^{\sharp, 2}(X)}, \\
\left\|G_{A} u\right\|_{L_{2, A}^{\sharp, 2}(X)} & \leq c\left(1+\nu_{0}[A]^{-1}\right)\left(1+\left\|F_{A}\right\|_{L^{2}(X)}\right)\|u\|_{L^{\sharp, 2}(X)} .
\end{aligned}
$$

Proof. The first and second assertions follow from Lemma 5.3, the fact that $\nabla_{A}^{*} \nabla_{A} G_{A} u=u$ for $u \in\left(\operatorname{Ker} \nabla_{A}^{*} \nabla_{A}\right)^{\perp}$, and the eigenvalue estimate $\|u\|_{L^{2}} \leq$ $\nu_{0}[A]^{-1}\left\|\nabla_{A}^{*} \nabla_{A} u\right\|_{L^{2}}$, while the third assertion follows from the first and Lemma 5.5.

5.2. Elliptic estimates for $d_{A}^{+}+d_{A}^{*}$. To illustrate their application and to point to possible extensions, we note that the estimates of Section 5.1 for the covariant Laplacian $\nabla_{A}^{*} \nabla_{A}=d_{A}^{*} d_{A}$ on $\Omega^{0}(E)$ naturally extend to give estimates for the covariant Laplacians $\nabla_{A}^{*} \nabla_{A}$ on $\Omega^{\ell}(E)=\Omega^{0}\left(\Lambda^{\ell} \otimes E\right)$. Estimates for $\nabla_{A}^{*} \nabla_{A}$ on $\Omega^{0}\left(\Lambda^{1} \otimes E\right)$ and $\Omega^{0}\left(\Lambda^{+} \otimes E\right)$ are of particular interest since these can in turn be profitably compared (via the Bochner-Weitzenböck formulas [10, Equations (6.25) \& (6.26)], as in [24]) with the remaining Laplacians defined by the elliptic deformation complex for the anti-self-dual equation [5], [10], namely $d_{A} d_{A}^{*}+d_{A}^{+, *} d_{A}^{+}$on $\Omega^{0}\left(\Lambda^{1} \otimes \mathfrak{g}_{E}\right)$ and $d_{A}^{+} d_{A}^{+, *}$ on $\Omega^{0}\left(\Lambda^{+} \otimes \mathfrak{g}_{E}\right)$. Indeed, if $B_{1}$ and $B_{+}$are the Levi-Civita connections on $\Lambda^{1}$ and $\Lambda^{+}$induced by the Levi-Civita connection on $T X$ for the metric $g$, then the curvature " $F_{A}$ " in the estimates of the preceding subsection is simply replaced by $[\mathbf{1 2}$, p. 165]

$$
\begin{aligned}
F_{B_{1} \otimes A} & =F_{B_{1}} \otimes \operatorname{id}_{\mathfrak{g}_{E}}+\mathrm{id}_{\Lambda^{1}} \otimes F_{A}, \\
F_{B_{+} \otimes A} & =F_{B_{+}} \otimes \operatorname{id}_{\mathfrak{g}_{E}}+\operatorname{id}_{\Lambda^{+}} \otimes F_{A},
\end{aligned}
$$

where $F_{B_{1}}$ and $F_{B_{+}}$are expressed in terms of the Riemann curvature tensor $\mathrm{Rm}$ and where we abuse notation slightly and denote the connections on $E$ and $\mathfrak{g}_{E}$ both by $A$. (See [10, Appendix C] and [11, Appendix II].) In the interests of brevity we shall confine our attention to the case of $L_{\ell}^{p}$ estimates with $p=2$, though the methods can be modified to obtain estimates for $p \neq 2$ (some work is required-see [5, p. 426] for hints). 
In order to compute the required elliptic estimates for $d_{A}^{+}$we will need the Bochner-Weitzenböck formulas,

$$
\begin{aligned}
d_{A} d_{A}^{*}+2 d_{A}^{*} d_{A}^{+} & =\nabla_{A}^{*} \nabla_{A}+\{\mathrm{Ric}, \cdot\}-2\left\{F_{A}^{-}, \cdot\right\}, \\
2 d_{A}^{+} d_{A}^{*} & =\nabla_{A}^{*} \nabla_{A}-2\left\{W^{+}, \cdot\right\}+\frac{R}{3}+\left\{F_{A}^{+}, \cdot\right\},
\end{aligned}
$$

for the Laplacians on $\Omega^{1}\left(\mathfrak{g}_{E}\right)$ and $\Omega^{+}\left(\mathfrak{g}_{E}\right)$ [10, Equations (6.25) \& (6.26)]; here, Ric, $W^{+}$, and $R$ are the Ricci, self-dual Weyl, and scalar curvatures of the Riemannian metric $g$ on $X$. In applications to the degeneration of anti-self-dual or "almost anti-self-dual" connections $A$ as in [20], [22], $[\mathbf{2 3}],[\mathbf{2 4}]$, we can usually arrange to have a uniform $L^{\infty}$ bound on $F_{A}^{+}$, but not a uniform $L^{p}$ bound on $F_{A}^{-}$when $p>2$. We derive estimates in the remainder of this subsection with such applications and assumptions in view. To illustrate the nature of the difficulty we first derive a naive $L_{1, A}^{2}$ estimate for $a \in L_{1}^{2}\left(\mathfrak{g}_{E}\right)$ in terms of the $L^{2}$ norm of $\left(d_{A}^{*}+d_{A}^{+}\right) a$ :

Lemma 5.7. Let $X$ be a closed, oriented four-manifold with metric $g$. Then there is a constant $c$ with the following significance. Let $E$ be a Riemannian vector bundle over $X$ and let $A$ be an orthogonal $L_{4}^{2}$ connection on $E$ with curvature $F_{A}$. Then for any $a \in L_{1}^{2}\left(\Lambda^{1} \otimes \mathfrak{g}_{E}\right)$,

$$
\begin{aligned}
\|a\|_{L_{1, A}^{2}(X)} \leq & \sqrt{2}\left\|\left(d_{A}^{*}+d_{A}^{+}\right) a\right\|_{L^{2}(X)} \\
& +c\left(1+\left\|F_{A}^{-}\right\|_{C^{0}(X)}\right)^{1 / 2}\|a\|_{L^{2}(X)} .
\end{aligned}
$$

If a is $L^{2}$-orthogonal to $\operatorname{Ker} d_{A}^{+}$, so that $a=d_{A}^{*} v$ for some $v \in L_{2}^{2}\left(\Lambda^{+} \otimes \mathfrak{g}_{E}\right)$, then

$$
\begin{aligned}
\left\|d_{A}^{*} v\right\|_{L_{1, A}^{2}(X)} \leq & \sqrt{2}\left\|d_{A}^{+} d_{A}^{*} v\right\|_{L^{2}(X)}+c\left(1+\left\|F_{A}^{-}\right\|_{C^{0}(X)}\right)^{1 / 2}\|v\|_{L^{2}(X)} \\
& +\left\|F_{A}^{+}\right\|_{C^{0}(X)}\|v\|_{L^{2}(X)} .
\end{aligned}
$$

Proof. From the Bochner-Weitzenböck formula for $d_{A} d_{A}^{*}+2 d_{A}^{*} d_{A}^{+}$in (5.6) and integration by parts, we have:

$$
\begin{aligned}
\left\|\nabla_{A} a\right\|_{L^{2}}^{2} & =\left(\nabla_{A}^{*} \nabla_{A} a, a\right) \\
& =\left(d_{A} d_{A}^{*} a, a\right)+2\left(d_{A}^{*} d_{A}^{+} a, a\right)-(\{\text { Ric }, a\}, a)+2\left(\left\{F_{A}^{-}, a\right\}, a\right) \\
& \leq\left\|d_{A}^{*} a\right\|_{L^{2}}^{2}+2\left\|d_{A}^{+} a\right\|_{L^{2}}^{2}+c\left(1+\left\|F_{A}^{-}\right\|_{C^{0}}\right)\|a\|_{L^{2}}^{2}
\end{aligned}
$$

which gives (5.8). If $a=d_{A}^{*} v$, then $d_{A}^{*} d_{A}^{*} v=\left(d_{A}^{+} d_{A}\right)^{*} v=\left(F_{A}^{+}\right)^{*} v$, so that

$$
\left\|d_{A}^{*} d_{A}^{*} v\right\|_{L^{2}} \leq\left\|F_{A}^{+}\right\|_{C^{0}}\|v\|_{L^{2}} .
$$

Thus, (5.9) follows from (5.8) and the above inequality.

Since $d_{A}^{*}+d_{A}^{+}$is an elliptic operator, estimates of the above form follow from the general theory of linear elliptic operators. However, the preceding elementary derivation using the Bochner-Weitzenböck formula gives us a 
constant whose dependence on the curvature terms $F_{A}^{-}$and $F_{A}^{+}$is made explicit. In particular, we see that the estimate is only useful when we have a uniform $C^{0}$ bound on $F_{A}^{-}$independent of $A$, which is not possible when $A$ bubbles. At the cost of introducing a slightly stronger norm than the $L^{2}$ norm on the right hand side of the estimate above, we can derive an $L_{1, A}^{2}$ bound for $a=d_{A}^{*} v$ with an estimate constant depending on $\left\|F_{A}^{-}\right\|_{L^{2}(X)}$ rather than $\left\|F_{A}^{-}\right\|_{C^{0}(X)}$. Specifically, Equation (5.5) and Lemma 5.5 give the following $L_{2, A}^{2}$ estimates for sections of $\Lambda^{+} \otimes \mathfrak{g}_{E}$ :

Lemma 5.8. Continue the hypotheses of Lemma 5.7. Then the following estimate holds for any $v \in L_{2}^{\sharp, 2}\left(\Lambda^{+} \otimes \mathfrak{g}_{E}\right)$ :

$$
\|v\|_{L_{2, A}^{2}(X)}+\|v\|_{C^{0}(X)} \leq c\left(1+\left\|F_{A}\right\|_{L^{2}(X)}\right)\left(\left\|\nabla_{A}^{*} \nabla_{A} v\right\|_{L^{\sharp, 2}(X)}+\|v\|_{L^{2}(X)}\right) .
$$

We now replace the covariant Laplacian $\nabla_{A}^{*} \nabla_{A}$ in the estimates of Lemma 5.8 by the Laplacian $d_{A}^{+} d_{A}^{*}$ via the Bochner formula (5.7) to give:

Lemma 5.9. Continue the hypotheses of Lemma 5.8. Then there is a positive constant $\varepsilon=\varepsilon(c)$ such that the following holds. If $\left\|F_{A}^{+}\right\|_{L^{\sharp, 2}(X)}<\varepsilon$, then

$$
\|v\|_{L_{2, A}^{2}(X)}+\|v\|_{C^{0}(X)} \leq c\left(1+\left\|F_{A}\right\|_{L^{2}(X)}\right)\left(\left\|d_{A}^{+} d_{A}^{*} v\right\|_{L^{\sharp, 2}(X)}+\|v\|_{L^{2}(X)}\right) .
$$

Proof. From (5.5) and Lemma 4.3 we have

$$
\left\|\nabla_{A}^{*} \nabla_{A} v\right\|_{L^{\sharp, 2}} \leq 2\left\|d_{A}^{+} d_{A}^{*} v\right\|_{L^{\sharp, 2}}+c\|v\|_{L^{\sharp, 2}}+c\left\|F_{A}^{+}\right\|_{L^{\sharp, 2}}\|v\|_{C^{0}} .
$$

Combining the preceding estimate with that of Lemma 5.8, together with the embedding and interpolation inequalities $\|v\|_{L^{\sharp}} \leq c\|v\|_{L^{4}} \leq c\|v\|_{L^{2}}^{1 / 2}\|v\|_{C^{0}}^{1 / 2}$, and using rearrangement with the last term yields the desired bound. In particular, by choosing $\varepsilon(c)$ small enough that $c\left\|F_{A}^{+}\right\|_{L^{\sharp, 2}}\|v\|_{C^{0}} \leq 1 / 2$, we may use rearrangement to bring the right-hand term $\|v\|_{C^{0}}$ to the left-hand side.

Since $\left\|d_{A}^{*} v\right\|_{L_{1, A}^{2}} \leq\|v\|_{L_{2, A}^{2}}$, Lemma 5.9 yields an $L_{1, A}^{2}$ estimate for $d_{A}^{*} v$ :

Corollary 5.10. Continue the hypotheses of Lemma 5.9. Then:

$$
\left\|d_{A}^{*} v\right\|_{L_{1, A}^{2}(X)} \leq c\left(1+\left\|F_{A}\right\|_{L^{2}(X)}\right)\left(\left\|d_{A}^{+} d_{A}^{*} v\right\|_{L^{\sharp, 2}(X)}+\|v\|_{L^{2}(X)}\right) .
$$

Note that if $a \in \Omega^{1}\left(\mathfrak{g}_{E}\right)$ is $L^{2}$-orthogonal to $\operatorname{Ker} d_{A}^{+}$, so that $a=d_{A}^{*} v$ for some $v \in \Omega^{+}\left(\mathfrak{g}_{E}\right)$, and $\operatorname{Ker} d_{A}^{+} d_{A}^{+, *}=0$, then the estimate of Corollary 5.10 can be written in the more familiar form

$$
\|a\|_{L_{1, A}^{2}(X)} \leq c\left(1+\left\|F_{A}\right\|_{L^{2}(X)}\right)\left(\left\|d_{A}^{+} a\right\|_{L^{\sharp, 2}(X)}+\nu_{2}[A]^{-1 / 2}\|a\|_{L^{2}(X)}\right),
$$

where we make use of the eigenvalue estimate $\|v\|_{L^{2}} \leq \nu_{2}[A]^{-1 / 2}\left\|d_{A}^{*} v\right\|_{L^{2}}$; the term $d_{A}^{+} a$ above can be replaced by $\left(d_{A}^{+}+d_{A}^{*}\right) a$ without changing the estimate constants. Here, $\nu_{2}[A]$ is the least positive eigenvalue of the Laplacian $d_{A}^{+} d_{A}^{+, *}$. 


\section{Existence of gauge transformations via the method of continuity.}

In this section we complete the proof of Theorem 1.1, and hence the proof of Theorem 1.2, using the method of continuity. The strategy broadly follows that of Uhlenbeck's proof of Theorem 2.1 in [26]. The main new technical difficulty, not present in [26], is the need to compare distances in the Coulomb-gauge slice $\mathbf{S}_{A_{0}} \subset \mathcal{A}_{E}^{k}$ through the connection $A_{0}$ and gaugeinvariant distances in $\mathcal{B}_{E}^{k}$ from the point $\left[A_{0}\right]$. It is at this stage of the method of continuity (in proving openness - see Lemma 6.6) - that we need to employ the special norms and Green's operator estimates described in Sections 4 and 5 in order to achieve the requisite $C^{0}$ control of gauge transformations; the proof of closedness works, as one would expect, with standard Sobolev $L^{4}$ and $L_{1}^{2}$ norms. In [26], the $L^{2}$ norm of the curvature $F_{A}$ essentially serves as a gauge-invariant $L_{1}^{2}$ measure of distance from $[A]$ to $[\Gamma]$, where $\Gamma$ is the product connection on the product $G$ bundle over the unit ball. Our goal in this section is to prove:

Theorem 6.1. Let $X$ be a closed, smooth four-manifold with metric $g$ and let $G$ be a compact Lie group. Then there are positive constants $c, z$ with the following significance. Let $E$ be a $G$ bundle over $X$ and suppose that $k \geq 2$ is an integer. Given a point $\left[A_{0}\right]$ in $\mathcal{B}_{E}^{k}$, let $\nu_{0}\left[A_{0}\right]$ be the least positive eigenvalue of the Laplacian $\nabla_{A_{0}}^{*} \nabla_{A_{0}}$ on $\Omega^{0}\left(\mathfrak{g}_{E}\right)$ and set $K_{0}=(1+$ $\left.\nu_{0}\left[A_{0}\right]^{-1}\right)\left(1+\left\|F_{A_{0}}\right\|_{L^{2}}\right)$. Let $\varepsilon_{1}$ be a constant satisfying $0<\varepsilon_{1} \leq z K_{0}^{-2}(1+$ $\left.\nu_{0}\left[A_{0}\right]^{-1 / 2}\right)^{-1}$. Then the following hold:

(1) For any $[A] \in \mathcal{B}_{E}^{k}$ with $\operatorname{dist}_{L_{1, A_{0}}^{\sharp, 2}}\left([A],\left[A_{0}\right]\right)<\varepsilon_{1}$, then $u \in \mathcal{G}_{E}^{k+1}$ exists such that

(a) $d_{A_{0}}^{*}\left(u(A)-A_{0}\right)=0$,

(b) $\left\|u(A)-A_{0}\right\|_{L^{2 \sharp, 4}} \leq c K_{0} \operatorname{dist}_{\mathcal{L}_{1, A_{0}}^{\sharp, 2}}\left([A],\left[A_{0}\right]\right)$.

(2) For any $[A] \in \mathcal{B}_{E}^{k}$ with $\operatorname{dist}_{L_{1, A_{0}}^{\sharp, 2}}\left([A],\left[A_{0}\right]\right)<\varepsilon_{1}$, then $u \in \mathcal{G}_{E}^{k+1}$ exists such that

(a) $d_{A_{0}}^{*}\left(u(A)-A_{0}\right)=0$,

(b) $\left\|u(A)-A_{0}\right\|_{L^{2 \sharp, 4}} \leq c K_{0} \operatorname{dist}_{\mathcal{L}_{1, A_{0}}^{\sharp, 2}}\left([A],\left[A_{0}\right]\right)$,

(c) $\left\|u(A)-A_{0}\right\|_{L_{1, A_{0}}^{2}} \leq c K_{0} \operatorname{dist}_{L_{1, A_{0}}^{\sharp, 2}}\left([A],\left[A_{0}\right]\right)$.

Our first proof of Theorem 6.1, via the method of continuity, occupies Sections 6.1, 6.2 and 6.3. A rather different proof, via a direct application of the inverse function theorem using $L_{2}^{\sharp, 2}$ gauge transformations, is given in Section 8.

6.1. Distance functions on the quotient space. Our first task is to verify the existence of minimizing gauge transformations $u \in \mathcal{G}_{E}^{k+1}$ for the 
family of distance functions on $\mathcal{B}_{E}^{k}$ defined above: This is established in Lemma 6.3 and the proof uses the following version of Uhlenbeck's weak compactness theorem.

Proposition 6.2. Let $X$ be a closed, smooth, Riemannian four-manifold, let $G$ be a compact Lie group, let $M$ be a positive constant, let $A_{0}$ be an $L_{2}^{2}$ connection on a $G$ bundle $E$ over $X$. If $\left\{A_{\alpha}\right\}$ is a sequence of $L_{2}^{2}$ connections on $E$ such that $\left\|F_{A_{\alpha}}\right\|_{L_{1, A_{0}}^{2}} \leq M$, then there is a subsequence $\left\{\alpha^{\prime}\right\} \subset\{\alpha\}$ and a sequence of $L_{3}^{2}$ gauge transformations $\left\{u_{\alpha^{\prime}}\right\}$ such that $u_{\alpha^{\prime}}\left(A_{\alpha^{\prime}}\right)$ converges weakly in $L_{2, A_{0}}^{2}$ and strongly in $L_{1, A_{0}}^{p}$, for $1 \leq p<4$, to an $L_{2}^{2}$ connection $A_{\infty}$ on $E$.

Proof. From the Sobolev embedding $L_{1}^{2} \subset L^{p}, 2<p<4$, we obtain a uniform $L^{p}$ bound $\left\|F_{A_{\alpha}}\right\|_{L^{p}} \leq c M$ and so, according to [26, Theorem 3.6], there is a subsequence $\left\{\alpha^{\prime}\right\} \subset\{\alpha\}$ and a sequence of $L_{2}^{p}$ gauge transformations $\left\{u_{\alpha^{\prime}}\right\}$ such that $u_{\alpha^{\prime}}\left(A_{\alpha^{\prime}}\right)$ converges weakly in $L_{1, A_{0}}^{p}$ to an $L_{1}^{p}$ connection $A_{\infty}$ on $E$. The stronger conclusion above is obtained simply by reworking the proof of Theorem 3.6 in [26], using the following local estimate for the connections $A_{\alpha}$ over small balls $B \subset X$. Theorem 2.1 of [26] provides a sequence of local trivializations $v_{\alpha}:\left.P\right|_{B} \rightarrow B \times G$ such that $a_{\alpha}=v_{\alpha}\left(A_{\alpha}\right)-\Gamma$ satisfies $d^{*} a_{\alpha}=0$ and

$$
\left\|a_{\alpha}\right\|_{L_{1}^{p}(B)} \leq c\left\|F_{A_{\alpha}}\right\|_{L^{p}(B)}, \quad 2 \leq p<4,
$$

where $\Gamma$ is the product connection. Now $F_{A_{\alpha}}=d a_{\alpha}+a_{\alpha} \wedge a_{\alpha}$, so

$$
\begin{aligned}
\left\|a_{\alpha}\right\|_{L_{2}^{2}(B)} & \leq\left\|d a_{\alpha}\right\|_{L_{1}^{2}(B)}+\left\|a_{\alpha}\right\|_{L_{1}^{2}(B)} \\
& \leq\left\|a_{\alpha} \wedge a_{\alpha}\right\|_{L_{1}^{2}(B)}+\left\|F_{A_{\alpha}}\right\|_{L_{1}^{2}(B)}+\left\|a_{\alpha}\right\|_{L_{1}^{2}(B)} .
\end{aligned}
$$

Now, using the multiplication $L^{6} \times L^{3} \rightarrow L^{2}$, the embeddings $L_{1}^{3} \subset L_{1}^{12 / 5} \subset$ $L^{6}$ and $d\left(a_{\alpha} \wedge a_{\alpha}\right)=d a_{\alpha} \wedge a_{\alpha}-a_{\alpha} \wedge d a_{\alpha}$, we have

$$
\left\|d\left(a_{\alpha} \wedge a_{\alpha}\right)\right\|_{L^{2}} \leq c\left\|d a_{\alpha}\right\|_{L^{3}}\left\|a_{\alpha}\right\|_{L^{6}} \leq c\left\|a_{\alpha}\right\|_{L_{1}^{3}}^{2}
$$

while $\left\|a_{\alpha} \wedge a_{\alpha}\right\|_{L^{2}} \leq\left\|a_{\alpha}\right\|_{L^{4}(B)}^{2} \leq c\left\|a_{\alpha}\right\|_{L_{1}^{2}(B)}^{2}$. Hence, we obtain

$$
\begin{aligned}
\left\|a_{\alpha}\right\|_{L_{2}^{2}(B)} & \leq c\left\|F_{A_{\alpha}}\right\|_{L_{1}^{2}(B)}+c\left\|a_{\alpha}\right\|_{L_{1}^{3}(B)}^{2}+\left\|a_{\alpha}\right\|_{L_{1}^{2}(B)} \\
& \leq c\left\|F_{A_{\alpha}}\right\|_{L_{1}^{2}(B)}\left(1+\left\|F_{A_{\alpha}}\right\|_{L_{1}^{2}(B)}\right) \\
& \leq c\left\|F_{A_{\alpha}}\right\|_{L_{1, A_{0}}^{2}(B)}\left(1+\left\|F_{A_{\alpha}}\right\|_{L_{1, A_{0}}^{2}(B)}\right)\left(1+\left\|A_{0}-\Gamma\right\|_{L_{1}^{2}}\right) .
\end{aligned}
$$

In particular, the sequence of Coulomb-gauge, local connection matrices $\left\{a_{\alpha}\right\}$ is bounded in $L_{2}^{2}(B)$, so we can extract a weakly $L_{2}^{2}(B)$-convergent and strongly $L_{1}^{p}(B)$-convergent subsequence, via the compactness of embedding $L_{2}^{2}(B) \subset L_{1}^{p}(B)$ when $1 \leq p<4$. The patching argument used to complete the proof of Uhlenbeck's theorem now proceeds exactly as in [26] to give the desired conclusion. 
The proposition is used to extract the desired convergence in the next lemma.

Lemma 6.3. For any points $\left[A_{0}\right],[A]$ in $\mathcal{B}_{E}^{k}$ there are gauge transformations such that the following equalities hold:

$$
\begin{aligned}
\operatorname{dist}_{L^{4}}\left([A],\left[A_{0}\right]\right) & =\left\|u(A)-A_{0}\right\|_{L^{4}(X)}, \quad u \in \mathcal{G}_{E}^{3} \\
\operatorname{dist}_{\mathcal{L}_{1, A_{0}}^{\sharp, 2}}\left([A],\left[A_{0}\right]\right) & =\left\|v(A)-A_{0}\right\|_{\mathcal{L}_{1, A_{0}}^{\sharp, 2},}, \quad v \in \mathcal{G}_{E}^{3}, \\
\operatorname{dist}_{L_{1, A_{0}}^{\sharp, 2}}\left([A],\left[A_{0}\right]\right) & =\left\|w(A)-A_{0}\right\|_{L_{1, A_{0}}^{\sharp, 2}}, \quad w \in \mathcal{G}_{E}^{3}, \\
\operatorname{dist}_{L_{\ell, A_{0}}^{2}}\left([A],\left[A_{0}\right]\right) & =\left\|w_{\ell}(A)-A_{0}\right\|_{L_{\ell, A_{0}}^{2}}, \quad \ell=1 \text { and } 3 \leq \ell \leq k,
\end{aligned}
$$

where $w_{1} \in \mathcal{G}_{E}^{3}$ and $w_{\ell} \in \mathcal{G}_{E}^{\ell+1}$ in (4). The above distance functions (including the $\ell=2$ distance function in (4)) are continuous with respect to the quotient $L_{k}^{2}$ topology on $\mathcal{B}_{E}^{k}$.

Proof. Consider (1). Let $\left\{u_{\alpha}\right\}$ be a minimizing sequence in $\mathcal{G}_{E}^{k+1}$, so $\| u_{\alpha}(A)-$ $A_{0} \|_{L^{4}}$ converges to $\operatorname{dist}_{L^{4}}\left([A],\left[A_{0}\right]\right)$ as $\alpha \rightarrow \infty$. Setting $B_{\alpha}=u_{\alpha}(A)=$ $A-\left(d_{A} u_{\alpha}\right) u_{\alpha}^{-1} \in \mathcal{A}_{E}^{k}$, we see that $B_{\alpha} u_{\alpha}=A u_{\alpha}-d_{A} u_{\alpha}=A u_{\alpha}-d_{A_{0}} u_{\alpha}-$ $\left[A-A_{0}, u_{\alpha}\right]$, and thus

$$
d_{A_{0}} u_{\alpha}=u_{\alpha}\left(A-A_{0}\right)-\left(B_{\alpha}-A_{0}\right) u_{\alpha} .
$$

Therefore, as $\left\|u_{\alpha}\right\|_{C^{0}} \leq c(G)$, we have

$$
\left\|\nabla_{A_{0}} u_{\alpha}\right\|_{L^{4}} \leq c\left(\left\|A-A_{0}\right\|_{L^{4}}+\left\|B_{\alpha}-A_{0}\right\|_{L^{4}}\right),
$$

so the sequence $\left\{u_{\alpha}\right\} \subset L_{k+1}^{2}(\mathfrak{g l}(E))$ is bounded in $L_{1, A_{0}}^{4}(\mathfrak{g l}(E))$. So, passing to a subsequence, we may suppose that $\left\{u_{\alpha}\right\}$ converges weakly in $L_{1, A_{0}}^{4}(\mathfrak{g l}(E))$ and strongly in $L^{q}(\mathfrak{g l}(E))$, via the compact embedding $L_{1}^{4} \subset L^{q}$, for any $1 \leq q<\infty$, to a limit $u \in L_{1}^{4}(\mathfrak{g l}(E))$.

We also have $F_{B_{\alpha}}=F_{u_{\alpha}(A)}=u_{\alpha} F_{A} u_{\alpha}^{-1}$, so $\left\|F_{B_{\alpha}}\right\|_{L^{2}}=\left\|F_{A}\right\|_{L^{2}}$ and as $\nabla_{A_{0}} F_{B_{\alpha}}=\left(\nabla_{A_{0}} u_{\alpha}\right) \otimes F_{A} u_{\alpha}^{-1}+u_{\alpha}\left(\nabla_{A_{0}} F_{A}\right) u_{\alpha}^{-1}-u_{\alpha} F_{A} \otimes u_{\alpha}^{-1}\left(\nabla_{A_{0}} u_{\alpha}\right) u_{\alpha}^{-1}$, we see that

$$
\begin{aligned}
\left\|\nabla_{A_{0}} F_{B_{\alpha}}\right\|_{L^{2}} & \leq c\left(\left\|\nabla_{A_{0}} u_{\alpha}\right\|_{L^{4}}\left\|F_{A}\right\|_{L^{4}}+\left\|\nabla_{A_{0}} F_{A}\right\|_{L^{2}}\right) \\
& \leq c\left(1+\left\|u_{\alpha}\right\|_{L_{1, A_{0}}^{4}}\right)\left\|F_{A}\right\|_{L_{1, A_{0}}^{2}} .
\end{aligned}
$$

Hence, the sequence of $L_{k}^{2}$ connections $\left\{B_{\alpha}\right\}$ has curvature uniformly bounded in $L_{1, A_{0}}^{2}$ : Proposition 6.2 implies, after passing to a subsequence, that the sequence $\left\{B_{\alpha}\right\}$ converges weakly in $L_{2, A_{0}}^{2}$ and strongly in $L_{1, A_{0}}^{p}$, for $1 \leq p<4$, to an $L_{2}^{2} G$ connection $B$ on $E$. From (6.1) we obtain

$$
d_{A_{0}} u=u\left(A-A_{0}\right)-\left(B-A_{0}\right) u,
$$

a first-order linear elliptic equation in $u$ with $L_{2}^{2}$ coefficients. Therefore, $u \in L_{3}^{2}(\mathfrak{g l}(E))$ and $B=u(A)=A-\left(d_{A} u\right) u^{-1}$ lies in $\mathcal{A}_{E}^{2}$. It is not a priori 
clear that the limit $u$ actually lies in $\mathcal{G}_{E}^{3}$ (since the convergence was only weakly $L_{1, A_{0}}^{4}(\mathfrak{g l}(E))$ and strongly $\left.L^{q}(\mathfrak{g l}(E))\right)$ : However, the argument of the last paragraph in the proof of Lemma 4.2 .4 in [5, p. 130] applies (using the compactness of the structure group $G$ ) and shows that the limit gauge transformation $u$ lies in $\mathcal{G}_{E}^{3}$. Since $B_{\alpha}=u_{\alpha}(A)$ converges strongly in $L_{1, A_{0}}^{p}$ to $u(A)$ we now have

$$
\operatorname{dist}_{L^{4}}\left([A],\left[A_{0}\right]\right)=\lim _{\alpha \rightarrow \infty}\left\|u_{\alpha}(A)-A_{0}\right\|_{L^{4}}=\left\|u(A)-A_{0}\right\|_{L^{4}},
$$

as required in (1). The same argument proves Assertions (2) and (3) and Assertion (4) when $\ell=1$. The case $\ell \geq 3$ in (4) is straightforward as we can now apply Lemma 3.1 to obtain the desired convergence.

It remains to check $L_{k}^{2}$ continuity. We just consider (1), as the remaining cases are identical. If $\left[A_{\alpha}\right] \in \mathcal{B}_{E}^{k}$ is a sequence converging to $\left[A_{\infty}\right] \in \mathcal{B}_{E}^{k}$, then there is a sequence of gauge transformations $s_{\alpha} \in \mathcal{G}_{E}^{k+1}$ such that $s_{\alpha}\left(A_{\alpha}\right)$ converges in $L_{k, A_{0}}^{2}$ to $A_{\infty} \in \mathcal{A}_{E}^{k}$ and, in particular, in $L^{4}$. But then

$$
\begin{aligned}
& \left|\operatorname{dist}_{L^{4}}\left(\left[A_{\alpha}\right],\left[A_{0}\right]\right)-\operatorname{dist}_{L^{4}}\left(\left[A_{\infty}\right],\left[A_{0}\right]\right)\right| \\
& \quad=\left|\operatorname{dist}_{L^{4}}\left(\left[s_{\alpha}\left(A_{\alpha}\right)\right],\left[A_{0}\right]\right)-\operatorname{dist}_{L^{4}}\left(\left[A_{\infty}\right],\left[A_{0}\right]\right)\right| \\
& \quad \leq \operatorname{dist}_{L^{4}}\left(\left[s_{\alpha}\left(A_{\alpha}\right)\right],\left[A_{\infty}\right]\right) \leq\left\|s_{\alpha}\left(A_{\alpha}\right)-A_{\infty}\right\|_{L^{4}},
\end{aligned}
$$

and so

as desired.

$$
\lim _{\alpha \rightarrow \infty} \operatorname{dist}_{L^{4}}\left(\left[A_{\alpha}\right],\left[A_{0}\right]\right)=\operatorname{dist}_{L^{4}}\left(\left[A_{\infty}\right],\left[A_{0}\right]\right),
$$

6.2. Closedness. Let $\mathfrak{B} \subset \bar{B}_{\left[A_{0}\right]}^{1, \sharp, 2}(\varepsilon)$ be the subset of points $[A]$ such that there exists a gauge transformation $u \in \mathcal{G}_{E}^{k+1}$ satisfying the conclusions of Assertion (2) of Theorem 6.1 ; let $\mathfrak{B}^{*} \subset \bar{B}_{\left[A_{0}\right]}^{1, *, 2}(\varepsilon)$ be the subset of points $[A]$ such that there exists a gauge transformation $u \in \mathcal{G}_{E}^{k+1}$ satisfying the conclusions of Assertion (1). As in the proof of Theorem 2.1 in [26], we apply the method of continuity to show that $\mathfrak{B}^{*}=\bar{B}_{\left[A_{0}\right]}^{1, *, 2}(\varepsilon)$ and $\mathfrak{B}=\bar{B}_{\left[A_{0}\right]}^{1, \sharp, 2}(\varepsilon)$ for small enough $\varepsilon$. Not surprisingly, we have:

Lemma 6.4. The balls $\bar{B}_{\left[A_{0}\right]}^{1, *, 2}(\varepsilon)$ and $\bar{B}_{\left[A_{0}\right]}^{1, \sharp, 2}(\varepsilon)$ are connected.

Proof. If $[A] \in \bar{B}_{\left[A_{0}\right]}^{1, \sharp, 2}(\varepsilon)$, there is a gauge transformation $u \in \mathcal{G}_{E}^{k+1}$ such that

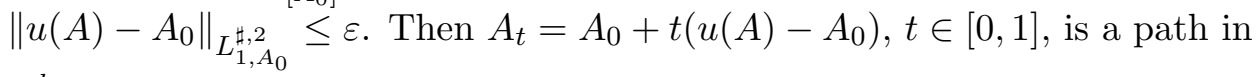
$\mathcal{A}_{E}^{k}$ joining $A_{0}$ to $u(A)$ and $\left\|A_{t}-A_{0}\right\|_{L_{1, A_{0}}^{\sharp, 2}}=t\left\|u(A)-A_{0}\right\|_{L_{1, A_{0}}^{\sharp, 2}} \leq t \varepsilon$, so the path $\left[A_{t}\right]$ lies in $\bar{B}_{\left[A_{0}\right]}^{1, \sharp, 2}(\varepsilon)$ and joins $\left[A_{0}\right]$ to $[A]$. Similarly for $\bar{B}_{\left[A_{0}\right]}^{1, *, 2}(\varepsilon)$.

Our task then reduces to showing that $\mathfrak{B}^{*}$ is an open and closed subspace of $\bar{B}_{\left[A_{0}\right]}^{1, *, 2}(\varepsilon)$ and that $\mathfrak{B}$ is an open and closed subspace of $\bar{B}_{\left[A_{0}\right]}^{1, \sharp, 2}(\varepsilon)$. First we consider closedness: 
Lemma 6.5. The subspaces $\mathfrak{B}^{*} \subset \bar{B}_{\left[A_{0}\right]}^{1, *, 2}(\varepsilon)$ and $\mathfrak{B} \subset \bar{B}_{\left[A_{0}\right]}^{1, \sharp, 2}(\varepsilon)$ are closed.

Proof. It suffices to consider the second assertion as the same argument yields the first. Suppose $\left[A_{\alpha}\right]$ is a sequence of points in $\mathfrak{B}$ which converges in $\mathcal{B}_{E}^{k}$ to a point $\left[B_{\infty}\right]$. We may suppose, without loss of generality, that $A_{\alpha} \in \mathcal{A}_{E}^{k}$ is the corresponding sequence of connections, representing the gauge-equivalence classes $\left[A_{\alpha}\right]$, which satisfy the defining conditions for $\mathfrak{B}$ :

$$
\begin{aligned}
& d_{A_{0}}^{*}\left(A_{\alpha}-A_{0}\right)=0, \\
&\left\|A_{\alpha}-A_{0}\right\|_{L^{2 \sharp, 4}} \leq c K_{0} \operatorname{dist}_{\mathcal{L}_{1, A_{0}}^{\sharp, 2}}\left(\left[A_{\alpha}\right],\left[A_{0}\right]\right), \\
&\left\|A_{\alpha}-A_{0}\right\|_{L_{1, A_{0}}^{2}} \leq c K_{0} \operatorname{dist}_{L_{1, A_{0}}^{\sharp, 2}}\left(\left[A_{\alpha}\right],\left[A_{0}\right]\right) .
\end{aligned}
$$

Since $\left[A_{\alpha}\right]$ converges in $\mathcal{B}_{E}^{k}$ to $\left[B_{\infty}\right]$, there is a sequence of gauge transformations $u_{\alpha} \in \mathcal{G}_{E}^{k+1}$ such that $B_{\alpha}:=u_{\alpha}\left(A_{\alpha}\right)$ converges in $L_{k, A_{0}}^{2}$ to $B_{\infty} \in \mathcal{A}_{E}^{k}$. Since $B_{\alpha}=u_{\alpha}\left(A_{\alpha}\right)$ and $d_{A_{0}}^{*}\left(A_{\alpha}-A_{0}\right)=0$, we have

$$
\begin{aligned}
d_{A_{0}} u_{\alpha}= & u_{\alpha}\left(A_{\alpha}-A_{0}\right)-\left(B_{\alpha}-A_{0}\right) u_{\alpha}, \\
d_{A_{0}}^{*} d_{A_{0}} u_{\alpha}=- & *\left(d_{A_{0}} u_{\alpha} \wedge *\left(A_{\alpha}-A_{0}\right)\right)-\left(d_{A_{0}}^{*}\left(B_{\alpha}-A_{0}\right)\right) u_{\alpha} \\
& -*\left(*\left(B_{\alpha}-A_{0}\right) \wedge d_{A_{0}} u_{\alpha}\right),
\end{aligned}
$$

and so, as $\left\|u_{\alpha}\right\|_{C^{0}} \leq 1$,

$$
\begin{gathered}
\left\|d_{A_{0}} u_{\alpha}\right\|_{L^{2 \sharp, 4}} \leq\left\|A_{\alpha}-A_{0}\right\|_{L^{2 \sharp, 4}}+\left\|B_{\alpha}-A_{0}\right\|_{L^{2 \sharp, 4}} \\
\left\|d_{A_{0}}^{*} d_{A_{0}} u_{\alpha}\right\|_{L^{\sharp, 2}} \leq\left\|d_{A_{0}} u_{\alpha}\right\|_{L^{2 \sharp, 4}}\left\|A_{\alpha}-A_{0}\right\|_{L^{2 \sharp, 4}}+\left\|d_{A_{0}}^{*} B_{\alpha}-A_{0}\right\|_{L^{\sharp, 2}} \\
\left\|B_{\alpha}-A_{0}\right\|_{L^{2 \sharp, 4}}\left\|d_{A_{0}} u_{\alpha}\right\|_{L^{2 \sharp, 4}} .
\end{gathered}
$$

Therefore, the sequence $u_{\alpha}$ is bounded in $L_{2, A_{0}}^{2}(\mathfrak{g l}(E))$ and so, passing to a subsequence, we may suppose that $u_{\alpha}$ converges weakly in $L_{2, A_{0}}^{2}(\mathfrak{g l}(E))$ (and strongly in $L_{1, A_{0}}^{p}$, for any $p<4$ via the compact embedding $L_{2}^{2} \subset L_{1}^{p}$ ) to a limit $u_{\infty} \in L^{\infty} \cap L_{2, A_{0}}^{2}(\mathfrak{g l}(E))$.

On the other hand, using $A_{\alpha}=u_{\alpha}^{-1}\left(B_{\alpha}\right)$, we have $\left\|F_{A_{\alpha}}\right\|_{L^{2}}=\left\|F_{B_{\alpha}}\right\|_{L^{2}}$ and the derivation of (6.2) gives

$$
\left\|\nabla_{A_{0}} F_{A_{\alpha}}\right\|_{L^{2}} \leq c\left(1+\left\|u_{\alpha}\right\|_{L_{1, A_{0}}^{4}}\right)\left\|F_{B_{\alpha}}\right\|_{L_{1, A_{0}}^{2}},
$$

so the sequence $A_{\alpha}$ has curvature uniformly bounded in $L_{1, A_{0}}^{2}$. Thus, after passing to a subsequence we may assume by Proposition 6.2 that the sequence $A_{\alpha}$ converges weakly in $L_{2, A_{0}}^{2}$ and strongly in $L_{1, A_{0}}^{p}, 2 \leq p<4$, to a limit $A_{\infty} \in \mathcal{A}_{E}^{2}$.

Taking weak limits in (6.5) and (6.6) yields

$$
d_{A_{0}} u_{\infty}=u_{\infty}\left(A_{\infty}-A_{0}\right)-\left(B_{\infty}-A_{0}\right) u_{\infty}
$$

The equation (6.7) is first order, linear, elliptic in $u_{\infty} \in L^{\infty} \cap L_{2}^{2}$ with $L_{2}^{2}$ coefficients. Hence, $u_{\infty}$ is in $L_{3}^{2}(\mathfrak{g l}(E))$ and in particular, in $\mathcal{G}_{E}^{3}$, while 
$B_{\infty}=u_{\infty}\left(A_{\infty}\right)$. From (6.7) we see that

$$
A_{\infty}-A_{0}=u_{\infty}^{-1}\left(B_{\infty}-A_{0}\right) u_{\infty}+u_{\infty}^{-1} d_{A_{0}} u_{\infty}
$$

and so, as $d_{A_{0}}^{*}\left(A_{\infty}-A_{0}\right)=0$, we have

$$
d_{A_{0}}^{*}\left(u_{\infty}^{-1} d_{A_{0}} u_{\infty}+u_{\infty}^{-1}\left(B_{\infty}-A_{0}\right) u_{\infty}\right)=0 .
$$

This is a second-order elliptic equation for $u_{\infty} \in \mathcal{G}_{E}^{3}$ with $L_{k}^{2}$ coefficients: In particular, since $u_{\infty} \in L_{2}^{p}$ for $2 \leq p \leq 4$, a standard elliptic bootstrapping argument then implies that $u_{\infty} \in L_{k+1}^{2}$ (see, for example, the proof of Proposition 3.3 in [6]) and therefore $A_{\infty}=u_{\infty}^{-1}\left(B_{\infty}\right) \in \mathcal{A}_{E}^{k}$.

Now, taking weak limits in (6.4), we have

$$
\begin{aligned}
d_{A_{0}}^{*}\left(A_{\infty}-A_{0}\right) & =\lim _{\alpha \rightarrow \infty} d_{A_{0}}^{*}\left(A_{\alpha}-A_{0}\right)=0, \\
\left\|A_{\infty}-A_{0}\right\|_{L^{2 \sharp, 4}} & =\lim _{\alpha \rightarrow \infty}\left\|A_{\alpha}-A_{0}\right\|_{L^{2 \sharp, 4}} \leq \lim _{\alpha \rightarrow \infty} c K_{0} \operatorname{dist}_{\mathcal{L}_{1, A_{0}}^{\sharp, 2}}\left(\left[A_{\alpha}\right],\left[A_{0}\right]\right), \\
\left\|A_{\alpha}-A_{0}\right\|_{L_{1, A_{0}}^{\sharp, 2}} & =\lim _{\alpha \rightarrow \infty}\left\|A_{\alpha}-A_{0}\right\|_{L_{1, A_{0}}^{\sharp, 2}} \leq \lim _{\alpha \rightarrow \infty} c K_{0} \operatorname{dist}_{L_{1, A_{0}}^{\sharp, 2}}\left(\left[A_{\alpha}\right],\left[A_{0}\right]\right) .
\end{aligned}
$$

Moreover, as $B_{\infty}=u_{\infty}\left(A_{\infty}\right)$ and $u_{\infty} \in \mathcal{G}_{E}^{k+1}$,

$$
\begin{gathered}
\lim _{\alpha \rightarrow \infty} \operatorname{dist}_{\mathcal{L}_{1, A_{0}}^{\sharp, 2}}\left(\left[A_{\alpha}\right],\left[A_{0}\right]\right)=\operatorname{dist}_{\mathcal{L}_{1, A_{0}}^{\sharp, 2}}\left(\left[B_{\infty}\right],\left[A_{0}\right]\right)=\operatorname{dist}_{\mathcal{L}_{1, A_{0}}^{\sharp, 2}}\left(\left[A_{\infty}\right],\left[A_{0}\right]\right), \\
\lim _{\alpha \rightarrow \infty} \operatorname{dist}_{L_{1, A_{0}}^{\sharp, 2}}\left(\left[A_{\alpha}\right],\left[A_{0}\right]\right)=\operatorname{dist}_{L_{1, A_{0}}^{\sharp, 2}}\left(\left[B_{\infty}\right],\left[A_{0}\right]\right)=\operatorname{dist}_{L_{1, A_{0}}^{\sharp, 2}}\left(\left[A_{\infty}\right],\left[A_{0}\right]\right),
\end{gathered}
$$

where the $L_{k}^{2}$ continuity of the distance functions is given by Lemma 6.3. Therefore, $\left[B_{\infty}\right]=\left[A_{\infty}\right] \in \mathfrak{B}$. Thus, $\mathfrak{B}$ is closed in $\mathcal{B}_{E}^{k}$ and in particular, closed in $\bar{B}_{\left[A_{0}\right]}^{1, \sharp, 2}(\varepsilon)$, as desired.

6.3. Openness. We must first compare distances from the connection $A_{0}$ in the Coulomb slice through $A_{0}$ in $\mathcal{A}_{E}^{k}$ and gauge-invariant distances in $\mathcal{B}_{E}^{k}$ from the point $\left[A_{0}\right]$ :

Lemma 6.6. Let $(X, g)$ be a closed, smooth, Riemannian four-manifold. Then there are positive constants $c, z$ with the following significance. Let $K_{0}=\left(1+\nu_{0}\left[A_{0}\right]^{-1}\right)\left(1+\left\|F_{A_{0}}\right\|_{L^{2}}\right)$. If $A \in \mathcal{A}_{E}^{k}$ satisfies

- $d_{A_{0}}^{*}\left(A-A_{0}\right)=0$,

- $\left\|A-A_{0}\right\|_{L^{2 \sharp, 4}} \leq z K_{0}^{-1}$,

then the following hold:

(1) If $\operatorname{dist}_{\mathcal{L}_{1, A_{0}}^{\sharp, 2}}\left([A],\left[A_{0}\right]\right) \leq z K_{0}^{-1}$, then

$$
\left\|A-A_{0}\right\|_{L^{2 \sharp, 4}} \leq c K_{0} \operatorname{dist}_{\mathcal{L}_{1, A_{0}}^{\sharp, 2}}\left([A],\left[A_{0}\right]\right) ;
$$

(2) If $\operatorname{dist}_{L_{1, A_{0}}^{\sharp, 2}}\left([A],\left[A_{0}\right]\right) \leq z K_{0}^{-1}$, then

$$
\begin{aligned}
&\left\|A-A_{0}\right\|_{L^{2 \sharp, 4}} \leq c K_{0} \operatorname{dist}_{\mathcal{L}_{1, A_{0}}^{\sharp, 2}}\left([A],\left[A_{0}\right]\right), \\
&\left\|A-A_{0}\right\|_{L_{1, A_{0}}^{2}} \leq c K_{0} \operatorname{dist}_{L_{1, A_{0}}^{\sharp, 2}}\left([A],\left[A_{0}\right]\right) .
\end{aligned}
$$


Proof. Recall that for either distance function, minimizing gauge transformations in $\mathcal{G}_{E}^{3}$ exist by Lemma 6.3 ; for convenience, we denote both by $u \in \mathcal{G}_{E}^{3}$ although they need not a priori coincide. Since $B:=u(A)=$ $A-\left(d_{A} u\right) u^{-1} \in \mathcal{A}_{E}^{2}$, we have

$$
u(A)-A_{0}=u\left(A-A_{0}\right) u^{-1}-\left(d_{A_{0}} u\right) u^{-1} .
$$

Our task, in essence, is to estimate the second term on the right above. Rewriting this equality gives a first-order, linear elliptic equation in $u$ with $L_{2}^{2}$ coefficients:

$$
d_{A_{0}} u=u\left(A-A_{0}\right)-\left(B-A_{0}\right) u .
$$

Let $u_{0} \in L_{3}^{2}(\mathfrak{g l}(E))$ be the $L^{2}$ orthogonal projection of $u \in \mathcal{G}_{E}^{3} \subset L_{3}^{2}(\mathfrak{g l}(E))$ onto $\operatorname{Ker}\left(\left.d_{A_{0}}\right|_{L_{3}^{2}}\right)^{\perp}$, so $u=u_{0}+\gamma$, where $\gamma \in \operatorname{Ker} d_{A_{0}} \subset \Omega^{0}(\mathfrak{g l}(E))$. Thus, as $d_{A_{0}}^{*}\left(A-A_{0}\right)=0$ and $d_{A_{0}} u=d_{A_{0}} u_{0}$, we see that

$$
\begin{aligned}
d_{A_{0}}^{*} d_{A_{0}} u_{0}=- & *\left(d_{A_{0}} u \wedge *\left(A-A_{0}\right)\right)+u d_{A_{0}}^{*}\left(A-A_{0}\right) \\
& -\left(d_{A_{0}}^{*}\left(B-A_{0}\right)\right) u-*\left(*\left(B-A_{0}\right) \wedge d_{A_{0}} u\right) \\
=- & *\left(d_{A_{0}} u_{0} \wedge *\left(A-A_{0}\right)\right)-\left(d_{A_{0}}^{*}\left(B-A_{0}\right)\right) u \\
& -*\left(*\left(B-A_{0}\right) \wedge d_{A_{0}} u_{0}\right) .
\end{aligned}
$$

Therefore, using the bound $\|u\|_{C^{0}} \leq 1$ for any $u \in \mathcal{G}_{E}^{3}$ (as the representation for $G$ is orthogonal), we have

$$
\begin{aligned}
\left\|\Delta_{A_{0}}^{0} u_{0}\right\|_{L^{\sharp, 2}} \leq & \left\|d_{A_{0}} u_{0}\right\|_{L^{2 \sharp, 4}}\left\|A-A_{0}\right\|_{L^{2 \sharp, 4}}+\left\|d_{A_{0}}^{*}\left(B-A_{0}\right)\right\|_{L^{\sharp, 2}}\|u\|_{C^{0}} \\
& +\left\|B-A_{0}\right\|_{L^{2 \sharp, 4}}\left\|d_{A_{0}} u_{0}\right\|_{L^{2 \sharp, 4}} \\
\leq C & \left(\left\|A-A_{0}\right\|_{L^{2 \sharp, 4}}+\left\|B-A_{0}\right\|_{L^{2 \sharp, 4}}\right)\left\|d_{A_{0}}^{*} d_{A_{0}} u_{0}\right\|_{L^{\sharp, 2}} \\
& +\left\|d_{A_{0}}^{*}\left(B-A_{0}\right)\right\|_{L^{\sharp, 2}},
\end{aligned}
$$

where $C=c K_{0}$. Now $\left\|B-A_{0}\right\|_{L^{2 \sharp, 4}} \leq c\left\|B-A_{0}\right\|_{L_{1, A_{0}}^{2}}$ via the embed$\operatorname{ding} L_{1}^{2} \subset L^{2 \sharp, 4}$ of Lemma 4.1. For either $\operatorname{dist}_{\mathcal{L}_{1, A_{0}}^{\sharp, 2}}\left([A],\left[A_{0}\right]\right) \leq \frac{1}{4} C^{-1}$ or $\operatorname{dist}_{L_{1, A_{0}}^{\sharp, 2}}\left([A],\left[A_{0}\right]\right) \leq \frac{1}{4} C^{-1}$ and $\left\|A-A_{0}\right\|_{L^{2 \sharp, 4}} \leq \frac{1}{4} C^{-1}$, rearrangement yields

$$
\left\|\Delta_{A_{0}}^{0} u_{0}\right\|_{L^{\sharp, 2}} \leq 2\left\|d_{A_{0}}^{*}\left(B-A_{0}\right)\right\|_{L^{\sharp, 2}} .
$$

On the other hand, from Lemma 5.6 we have

$$
\begin{aligned}
&\left\|u_{0}\right\|_{L_{2, A_{0}}^{\sharp, 2}} \leq C\left\|\Delta_{A_{0}}^{0} u_{0}\right\|_{L^{\sharp, 2}}, \\
&\left\|u_{0}\right\|_{L_{1, A_{0}}^{4}} \leq C\left\|\Delta_{A_{0}}^{0} u_{0}\right\|_{L^{\sharp, 2}},
\end{aligned}
$$


where $C=c K_{0}$ and the second bound follows from the embedding $L_{2}^{2} \subset L_{1}^{4}$. So, combining (6.10) and (6.11) yields:

$$
\begin{aligned}
& \left\|u_{0}\right\|_{L_{2, A_{0}}^{\sharp, 2}} \leq C\left\|d_{A_{0}}^{*}\left(B-A_{0}\right)\right\|_{L^{\sharp, 2}}, \\
& \left\|u_{0}\right\|_{L_{1, A_{0}}^{4}} \leq C\left\|d_{A_{0}}^{*}\left(B-A_{0}\right)\right\|_{L^{\sharp, 2}} .
\end{aligned}
$$

Consequently, using $d_{A_{0}} u=d_{A_{0}} u_{0}$ and (6.9) rewritten in the form,

$$
u^{-1}\left(B-A_{0}\right) u=\left(A-A_{0}\right)-u^{-1} d_{A_{0}} u_{0},
$$

we obtain

$$
\begin{aligned}
\left\|A-A_{0}\right\|_{L^{2 \sharp, 4}} & \leq\left\|u^{-1}\left(B-A_{0}\right) u\right\|_{L^{2 \sharp, 4}}+\left\|u^{-1} d_{A_{0}} u_{0}\right\|_{L^{2 \sharp, 4}}, \\
\left\|A-A_{0}\right\|_{L_{1, A_{0}}^{2}} & \leq\left\|u^{-1}\left(B-A_{0}\right) u\right\|_{L_{1, A_{0}}^{2}}+\left\|u^{-1} d_{A_{0}} u_{0}\right\|_{L_{1, A_{0}}^{2}} .
\end{aligned}
$$

From (6.14) and (6.12), we see that

$$
\begin{aligned}
\left\|A-A_{0}\right\|_{L^{2 \sharp, 4}} & \leq\left\|B-A_{0}\right\|_{L^{2 \sharp, 4}}+\left\|d_{A_{0}} u_{0}\right\|_{L^{2 \sharp, 4}} \\
& \leq \operatorname{dist}_{\mathcal{L}_{1, A_{0}}^{\sharp, 2}}\left([A],\left[A_{0}\right]\right)+C\left\|d_{A_{0}}^{*}\left(B-A_{0}\right)\right\|_{L^{\sharp, 2}} \\
& \leq(1+C) \operatorname{dist}_{\mathcal{L}_{1, A_{0}}^{\sharp, 2}}\left([A],\left[A_{0}\right]\right),
\end{aligned}
$$

giving the desired $L^{2 \sharp, 4}$ estimate for $A-A_{0}$.

Considering the first term in (6.13), we have

$$
\begin{aligned}
\nabla_{A_{0}}\left(u^{-1}\left(B-A_{0}\right) u\right)= & -u^{-1}\left(\nabla_{A_{0}} u\right) u^{-1} \otimes\left(B-A_{0}\right) u \\
& +u^{-1}\left(\nabla_{A_{0}}\left(B-A_{0}\right)\right) u+u^{-1}\left(B-A_{0}\right) \otimes \nabla_{A_{0}} u,
\end{aligned}
$$

and so applying (6.12), noting that $\nabla_{A_{0}} u=\nabla_{A_{0}} u_{0}$ and $\|u\|_{C^{0}} \leq 1$, we have

$$
\begin{aligned}
\left\|\nabla_{A_{0}}\left(u^{-1}\left(B-A_{0}\right) u\right)\right\|_{L^{2}} & \leq\left\|\nabla_{A_{0}} u_{0}\right\|_{L^{4}}\left\|B-A_{0}\right\|_{L^{4}}+\left\|\nabla_{A_{0}}\left(B-A_{0}\right)\right\|_{L^{2}} \\
& \leq C \operatorname{dist}_{L_{1, A_{0}}^{\sharp, 2}}^{2}\left([A],\left[A_{0}\right]\right)+\operatorname{dist}_{L_{1, A_{0}}^{\sharp, 2}}\left([A],\left[A_{0}\right]\right) .
\end{aligned}
$$

Thus, if $\operatorname{dist}_{L_{1, A_{0}}^{\sharp, 2}}\left([A],\left[A_{0}\right]\right) \leq \frac{1}{4} C^{-1}$, say, we obtain

$$
\left\|\nabla_{A_{0}}\left(u^{-1}\left(B-A_{0}\right) u\right)\right\|_{L^{2}} \leq 2 \operatorname{dist}_{L_{1, A_{0}}^{\sharp, 2}}\left([A],\left[A_{0}\right]\right) .
$$

Similarly, considering the second term in (6.13), we have

$$
\nabla_{A_{0}}\left(u^{-1} d_{A_{0}} u_{0}\right)=-u^{-1}\left(\nabla_{A_{0}} u\right) u^{-1} \otimes d_{A_{0}} u+u^{-1} \nabla_{A_{0}} d_{A_{0}} u
$$

and therefore, by (6.12), we see that

$$
\begin{aligned}
\left\|\nabla_{A_{0}}\left(u^{-1} d_{A_{0}} u_{0}\right)\right\|_{L^{2}} & \leq\left\|\nabla_{A_{0}} u_{0}\right\|_{L^{4}}^{2}+\left\|\nabla_{A_{0}}^{2} u_{0}\right\|_{L^{2}} \\
& \leq C \operatorname{dist}_{L_{1, A_{0}}^{\sharp, 2}}\left([A],\left[A_{0}\right]\right)\left(1+C \operatorname{dist}_{L_{1, A_{0}}^{\sharp, 2}}\left([A],\left[A_{0}\right]\right)\right) .
\end{aligned}
$$


Provided dist $_{L_{1, A_{0}}^{\sharp, 2}}\left([A],\left[A_{0}\right]\right) \leq \frac{1}{4} C^{-1}$, we obtain

$$
\left\|\nabla_{A_{0}}\left(u^{-1} d_{A_{0}} u_{0}\right)\right\|_{L^{2}} \leq 2 C \operatorname{dist}_{L_{1, A_{0}}^{\sharp, 2}}\left([A],\left[A_{0}\right]\right) .
$$

Taking the $L^{2}$ norm of (6.13) and applying (6.12) to estimate the second term gives

$$
\begin{aligned}
\left\|A-A_{0}\right\|_{L^{2}} & \leq\left\|B-A_{0}\right\|_{L^{2}}+\left\|d_{A_{0}} u_{0}\right\|_{L^{2}} \\
& \leq \operatorname{dist}_{L_{1, A_{0}}^{\sharp, 2}}\left([A],\left[A_{0}\right]\right)+C\left\|d_{A_{0}}^{*}\left(B-A_{0}\right)\right\|_{L^{\sharp, 2}},
\end{aligned}
$$

and so

$$
\left\|A-A_{0}\right\|_{L^{2}} \leq(1+C) \operatorname{dist}_{L_{1, A_{0}}^{\sharp, 2}}\left([A],\left[A_{0}\right]\right) .
$$

Combining the estimates (6.15), (6.16), (6.17), and (6.18) yields

$$
\left\|A-A_{0}\right\|_{L_{1, A_{0}}^{2}} \leq 2(1+C) \operatorname{dist}_{L_{1, A_{0}}^{\sharp, 2}}\left([A],\left[A_{0}\right]\right),
$$

giving the desired $L_{1, A_{0}}^{2}$ estimate for $A-A_{0}$.

Naturally, a comparison - going in the other direction - of distances from $A_{0}$ in the Coulomb slice in $\mathcal{A}_{E}^{k}$ through $A_{0}$ and gauge-invariant distances in $\mathcal{B}_{E}^{k}$ from the point $\left[A_{0}\right]$ is elementary: If $A \in \mathbf{S}_{A_{0}}$ and $\left\|A-A_{0}\right\|_{L_{k, A_{0}}^{2}}<\delta$, say, then Lemma 4.1 implies that

$$
\begin{aligned}
& \operatorname{dist}_{\mathcal{L}_{1, A_{0}}^{\sharp, 2}}\left([A],\left[A_{0}\right]\right) \leq c\left\|A-A_{0}\right\|_{L_{k, A_{0}}^{2}}<c \delta, \quad k \geq 1, \\
& \operatorname{dist}_{L_{1, A_{0}}^{\sharp, 2}}\left([A],\left[A_{0}\right]\right) \leq c\left\|A-A_{0}\right\|_{L_{k, A_{0}}^{2}}<c \delta, \quad k \geq 2,
\end{aligned}
$$

for some positive constant $c(X, g, k)$. The observation is used in concluding that $\mathfrak{B}^{*}, \mathfrak{B}$ are open subspaces of $\bar{B}_{\left[A_{0}\right]}^{1, *, 2}\left(\varepsilon_{1}\right), \bar{B}_{\left[A_{0}\right]}^{1, \sharp, 2}\left(\varepsilon_{1}\right)$, respectively:

Lemma 6.7. Let $(X, g)$ be a closed, smooth, Riemannian four-manifold and let $G$ be a compact Lie group. Then there is a positive constant $z$ with the following significance. Let $K_{0}=\left(1+\nu_{0}\left[A_{0}\right]^{-1}\right)\left(1+\left\|F_{A_{0}}\right\|_{L^{2}}\right)$. If $\varepsilon_{1}<$ $z K_{0}^{-2}\left(1+\nu_{0}\left[A_{0}\right]^{-1 / 2}\right)^{-1}$, then:

- $\mathfrak{B}^{*}$ is an open subspace of $\bar{B}_{\left[A_{0}\right]}^{1, *, 2}\left(\varepsilon_{1}\right)$;

- $\mathfrak{B}$ is an open subspace of $\bar{B}_{\left[A_{0}\right]}^{1, \sharp, 2}\left(\varepsilon_{1}\right)$.

Proof. It suffices to consider the second assertion, as the argument for the first is identical. Suppose $[A] \in \mathfrak{B}$ and that $A \in \mathcal{A}_{E}^{k}$ is a representative satisfying the defining conditions for $\mathfrak{B}$. Then $A$ satisfies $d_{A_{0}}^{*}\left(A-A_{0}\right)=0$ and the estimates

$$
\begin{aligned}
\left\|A-A_{0}\right\|_{L^{2 \sharp, 4}} & \leq c_{0} K_{0} \operatorname{dist}_{\mathcal{L}_{1, A_{0}}^{\sharp, 2}}\left([A],\left[A_{0}\right]\right), \leq c_{0} K_{0} \varepsilon_{1} \\
\left\|A-A_{0}\right\|_{L_{1, A_{0}}^{2}} & \leq c_{0} K_{0} \operatorname{dist}_{L_{1, A_{0}}^{\sharp, 2}}\left([A],\left[A_{0}\right]\right) \leq c_{0} K_{0} \varepsilon_{1},
\end{aligned}
$$


while $\left\|A-A_{0}\right\|_{L^{2 \sharp, 4}} \leq c_{1}\left\|A-A_{0}\right\|_{L_{1, A_{0}}^{2}}$ via the Sobolev embedding $L_{1}^{2} \subset$ $L^{2 \sharp, 4}$ and Kato's inequality. Consequently, if $c_{1} c_{0} K_{0} \varepsilon_{1} \leq \frac{1}{2} \varepsilon_{0}$, then $A \in$ $\mathbf{B}_{A_{0}}^{4}\left(\varepsilon_{0}\right) \subset \mathcal{A}_{E}^{k}$ and we see that

$$
\bar{B}_{\left[A_{0}\right]}^{1, \sharp, 2}\left(\varepsilon_{1}\right) \subset \pi\left(\mathbf{B}_{\left[A_{0}\right]}^{4}\left(\varepsilon_{0}\right)\right) .
$$

Lemma 3.6 implies that the map $\pi: \mathbf{B}_{\left[A_{0}\right]}^{4}\left(\varepsilon_{0}\right) / \operatorname{Stab}_{A_{0}} \rightarrow \mathcal{B}_{E}^{k}$ given by $A^{\prime} \mapsto\left[A^{\prime}\right]$ is a local homeomorphism onto its image $\pi\left(\mathbf{B}_{\left[A_{0}\right]}^{4}\left(\varepsilon_{0}\right)\right)$ for any $\varepsilon_{0}<$ $z\left(1+\nu_{0}\left[A_{0}\right]^{-1 / 2}\right)^{-1}$. In particular, if $A^{\prime} \in \mathbf{B}_{\left[A_{0}\right]}^{4}\left(\varepsilon_{0}\right)$ and $\left\|A^{\prime}-A\right\|_{L_{k, A_{0}}^{2}}<\delta$, then $A^{\prime} \in B_{\left[A_{0}\right]}^{1, \sharp, 2}\left(\varepsilon_{1}\right) \subset \bar{B}_{\left[A_{0}\right]}^{1, \sharp, 2}\left(\varepsilon_{1}\right)$ for small enough $\delta$.

The embedding $L_{1}^{2} \subset L^{2 \sharp, 4}$ and Lemma 6.6 imply that if $\left\|A^{\prime}-A_{0}\right\|_{L_{1, A_{0}}^{2}} \leq$ $z K_{0}^{-1}$ and $\operatorname{dist}_{L_{1, A_{0}}^{\sharp, 2}}\left(\left[A^{\prime}\right],\left[A_{0}\right]\right) \leq z K_{0}^{-1}$, then

$$
\begin{aligned}
&\left\|A^{\prime}-A_{0}\right\|_{L^{2 \sharp, 4}} \leq c K_{0} \operatorname{dist}_{\mathcal{L}_{1, A_{0}}^{\sharp, 2}}\left(\left[A^{\prime}\right],\left[A_{0}\right]\right) \leq c K_{0} \varepsilon_{1}, \\
&\left\|A^{\prime}-A_{0}\right\|_{L_{1, A_{0}}^{2}} \leq c K_{0} \operatorname{dist}_{L_{1, A_{0}}^{\sharp, 2}}\left(\left[A^{\prime}\right],\left[A_{0}\right]\right) \leq c K_{0} \varepsilon_{1} .
\end{aligned}
$$

These inequalities are satisfied by $A$; moreover $\operatorname{dist}_{L_{1, A_{0}}^{\sharp, 2}}\left([A],\left[A_{0}\right]\right) \leq \varepsilon_{1}$ and $\left\|A-A_{0}\right\|_{L_{1, A_{0}}^{2}} \leq c_{0} K \varepsilon_{1}$. Require that $\varepsilon_{1} \leq \frac{1}{2} z K_{0}^{-1}$ and $c_{0} K_{0} \varepsilon_{1} \leq \frac{1}{2} z K_{0}^{-1}$, so $\varepsilon_{1} \leq \frac{1}{2} z \min \left\{1, c_{0}\right\} K_{0}^{-2}$. Hence, if $A^{\prime}$ is $L_{k, A_{0}}^{2}$-close enough to $A$ (where $k \geq 2$ ), we can ensure $\left[A^{\prime}\right]$ obeys the last two defining conditions for $\mathfrak{B}$ and so $\left[A^{\prime}\right] \in \mathfrak{B}$. Thus, $\mathfrak{B} \subset \bar{B}_{\left[A_{0}\right]}^{1, \sharp, 2}\left(\varepsilon_{1}\right)$ is open, as desired.

We can now conclude the proof of Theorem 6.1:

Proof of Theorem 6.1. Lemmas 6.5 and 6.7 imply that $\mathfrak{B}$ is an open and closed subset of the connected space $\bar{B}_{\left[A_{0}\right]}^{1, \sharp, 2}\left(\varepsilon_{1}\right)$, so $\mathfrak{B}=\bar{B}_{\left[A_{0}\right]}^{1, \sharp, 2}\left(\varepsilon_{1}\right)$. Similarly for $\mathfrak{B}^{*}$ and $\bar{B}_{\left[A_{0}\right]}^{1, *, 2}\left(\varepsilon_{1}\right)$ and hence the result follows.

Similarly, we conclude the proofs of our main theorems:

Proof of Theorem 1.1. Given Theorem 6.1, the only assertion left unaccounted for is the uniqueness of the gauge transformation $u \in \mathcal{G}_{E}^{k+1}$, modulo $\operatorname{Stab}_{A_{0}}$. But this follows from Lemma 3.7, just as in the paragraph immediately following the proof of that lemma.

Proof of Theorem 1.2. For the proof of Assertion (1), see the first paragraph of Section 1.3. The first inclusion in Assertion (2), namely $B_{\left[A_{0}\right]}^{1, \sharp, 2}\left(\varepsilon_{1}\right) \subset$ $B_{\left[A_{0}\right]}^{1, *, 2}\left(c_{1} \varepsilon_{1}\right)$, follows from the definition (1.3) of the two distance functions defining the balls (1.4) and the Sobolev embedding $L_{1}^{2} \subset L^{2 \sharp, 4}$ in Lemma 4.1. The second inclusion in Assertion (2), namely $B_{\left[A_{0}\right]}^{1, *, 2}\left(c_{1} \varepsilon_{1}\right) \subset \pi\left(\mathbf{B}_{A_{0}}^{4}\left(c_{2} K_{0} \varepsilon_{1}\right)\right)$, 
follows from the definition (1.4) of these balls and Assertion (1) in Theorem 1.1.

\section{Critical-exponent Sobolev norms and the group of gauge transformations.}

We now define an $L_{2}^{\sharp, 2}$ space of gauge transformations, by analogy with the definition of $\mathcal{G}_{E}^{k+1}$ when $k \geq 2$, and set

$$
\mathcal{G}_{E}^{2, \sharp, 2}:=\left\{u \in L_{2}^{\sharp, 2}(\mathfrak{g l}(E)): u \in G \text { a.e. }\right\} \subset L_{k}^{2}(\mathfrak{g l}(E)) .
$$

It is not entirely clear a priori that $\mathcal{G}_{E}^{2, \sharp 2}$ is a Banach Lie group. In the case of its counterpart, $\mathcal{G}_{E}^{k+1}$, the manifold structure follows from the fact that the exponential map

$$
\operatorname{Exp}: T_{\operatorname{id}_{E}} \mathcal{G}_{E}=\Omega^{0}\left(\mathfrak{g}_{E}\right) \rightarrow \mathcal{G}_{E}, \quad \zeta \mapsto \operatorname{Exp} \zeta,
$$

extends to a smooth map Exp $: L_{k+1}^{2}\left(\mathfrak{g}_{E}\right) \rightarrow L_{k+1}^{2}\left(\mathfrak{g}_{E}\right)$ and defines a system of smooth coordinate charts for $\mathcal{G}_{E}^{k+1}$. Here, Exp is defined pointwise at $u \in \mathcal{G}_{E}$ for $\zeta \in T_{\operatorname{id}_{E}} \mathcal{G}_{E}$ by setting

$$
\left(\operatorname{Exp}_{u} \zeta\right)(x):=\exp _{u(x)}(\zeta(x)), \quad x \in X,
$$

where $\exp : \mathfrak{g} \rightarrow G$ is the usual, $C^{\infty}$ exponential map for the Lie group $G$ on the right-hand side [10, Appendix A].

To verify that $\mathcal{G}_{E}^{2, \sharp 2}$ is in fact a Banach Lie group we will need estimates for the covariant derivatives of the exponential map. The estimates below follow by reworking the usual proof of the Sobolev lemma for left composition of Sobolev sections by smooth vector bundle maps [15, Lemma 9.9]. The difference here is that we keep track of the dependence of the constants on the geometric data: This precision is required for the implicit function argument in the next section in order to complete the proof of our slice theorem.

For $\chi, \zeta, \xi \in \Omega^{0}\left(\mathfrak{g}_{E}\right)$, the differentials

$$
\begin{aligned}
& (D \operatorname{Exp})_{\chi}: \Omega^{0}\left(\mathfrak{g}_{E}\right) \rightarrow T_{\operatorname{Exp} \chi} \mathcal{G}_{E}, \quad \zeta \mapsto(D \operatorname{Exp})_{\chi} \zeta, \\
& \left(D^{2} \operatorname{Exp}\right)_{\chi, \zeta}: \Omega^{0}\left(\mathfrak{g}_{E}\right) \rightarrow T_{\operatorname{Exp} \chi} \mathcal{G}_{E}, \quad \xi \mapsto\left(D^{2} \operatorname{Exp}\right)_{\chi, \zeta} \xi,
\end{aligned}
$$

are defined pointwise by setting

$$
\begin{aligned}
\left.(D \operatorname{Exp})_{\chi} \zeta\right|_{x} & =(D \exp )_{\chi(x)} \zeta(x), \\
\left.\left(D^{2} \operatorname{Exp}\right)_{\chi, \zeta} \xi\right|_{x} & =(D \exp )_{\chi(x), \zeta(x)} \xi(x),
\end{aligned}
$$

for any $x \in X$. When writing the differential $\left(D^{2} \operatorname{Exp}\right)_{\chi, \zeta}$ above, we have identified $T_{(D \exp )_{\chi} \zeta}\left(T_{\operatorname{Exp} \chi} \mathcal{G}_{E}\right)$ with $T_{\operatorname{Exp} \chi} \mathcal{G}_{E}$. 
The maps $(D \operatorname{Exp})_{\chi}: \Omega^{0}\left(\mathfrak{g}_{E}\right) \rightarrow \Omega^{0}\left(\mathfrak{g}_{E}\right)$ and $\left(D^{2} \operatorname{Exp}\right)_{\chi, \zeta}: \Omega^{0}\left(\mathfrak{g}_{E}\right) \rightarrow$ $\Omega^{0}\left(\mathfrak{g}_{E}\right)$ extend linearly to maps

$$
\begin{aligned}
& (D \operatorname{Exp})_{\chi}: C^{\infty}\left(\otimes^{\ell}\left(T^{*} X\right) \otimes \mathfrak{g}_{E}\right) \rightarrow C^{\infty}\left(\otimes^{\ell}\left(T^{*} X\right) \otimes \mathfrak{g}_{E}\right), \\
& \left(D^{2} \operatorname{Exp}\right)_{\chi}: C^{\infty}\left(\otimes^{\ell}\left(T^{*} X\right) \otimes \mathfrak{g}_{E}\right) \rightarrow C^{\infty}\left(\otimes^{\ell}\left(T^{*} X\right) \otimes \mathfrak{g}_{E}\right),
\end{aligned}
$$

for $\ell \geq 1$, by setting

$$
\begin{aligned}
(D \operatorname{Exp})_{\chi}(\theta \otimes \zeta) & =\theta \otimes(D \operatorname{Exp})_{\chi} \zeta, \\
\left(D^{2} \operatorname{Exp}\right)_{\chi, \zeta}(\theta \otimes \xi) & =\theta \otimes\left(D^{2} \operatorname{Exp}\right)_{\chi, \zeta} \xi,
\end{aligned}
$$

for $\theta \in \otimes^{\ell}\left(T^{*} X\right)$ and $\xi \in \Omega^{0}\left(\mathfrak{g}_{E}\right)$. As usual, we embed $\mathcal{G}_{E} \subset \Omega^{0}\left(\mathfrak{g}_{E}\right)$ in order to compute the covariant derivatives of sections $u \in \mathcal{G}_{E}$.

Lemma 7.1. Let $G$ be a compact Lie group. Then there is a positive constant $c(G)$ with the following significance. Let $X$ be a closed, smooth, Riemannian four-manifold. If $A$ is a $C^{\infty}$ connection on a $G$ bundle $E$, and $\chi \in \Omega^{0}\left(\mathfrak{g}_{E}\right)$, then we have pointwise bounds:

$$
\begin{aligned}
\left|\nabla_{A} e^{\chi}\right| & \leq\left|\nabla_{A} \chi\right|+c|\chi|\left|\nabla_{A} \chi\right|, \\
\left|\nabla_{A}^{2} e^{\chi}\right| & \leq c\left(|\chi|+\left|\nabla_{A} \chi\right|\right)\left|\nabla_{A} \chi\right|+c(1+|\chi|)\left|\nabla_{A}^{2} \chi\right|, \\
\left|\nabla_{A}^{*} \nabla_{A} e^{\chi}\right| & \leq c\left(|\chi|+\left|\nabla_{A} \chi\right|\right)\left|\nabla_{A} \chi\right|+c(1+|\chi|)\left|\nabla_{A}^{*} \nabla_{A} \chi\right| .
\end{aligned}
$$

Proof. We have

$$
\nabla_{A} e^{\chi}=\nabla_{A}(\operatorname{Exp} \chi)=(D \operatorname{Exp})_{\chi} \circ d_{A} \chi \in \Omega^{1}\left(\mathfrak{g}_{E}\right) .
$$

Since $(D \exp )_{0}=\operatorname{id}_{\mathbb{E}}$ and $\exp : \mathfrak{g} \rightarrow G$ is analytic, we have the pointwise bound $\left|(D \exp )_{\chi(x)}-\operatorname{id}_{\mathbb{E}}\right| \leq c(G)|\chi(x)|$ and thus a pointwise bound

$$
\left|(D \operatorname{Exp})_{\chi}-\mathrm{id}_{E}\right| \leq c|\chi|,
$$

noting that $(D \operatorname{Exp})_{0}=\operatorname{id}_{E}$. Therefore, we have

$$
\left|\nabla_{A} e^{\chi}\right| \leq\left|\nabla_{A} \chi\right|+c|\chi|\left|\nabla_{A} \chi\right|
$$

which gives the first assertion.

Define $\Phi(\chi, \zeta)=(D \operatorname{Exp})_{\chi}(\zeta) \in \Omega^{1}\left(\mathfrak{g}_{E}\right)$, for $\chi \in \Omega^{0}\left(\mathfrak{g}_{E}\right)$ and $\zeta \in \Omega^{1}\left(\mathfrak{g}_{E}\right)$, noting that $\Phi$ is nonlinear in $\chi$, but linear in $\zeta$. Thus,

$$
\nabla_{A}^{2} u=\left(D_{1} \Phi\right)_{\left(\chi, \nabla_{A} \chi\right)}\left(\nabla_{A} \chi\right)+\left(D_{2} \Phi\right)_{\chi}\left(\nabla_{A}^{2} \chi\right)
$$

where $D_{i} \Phi, i=1,2$, denote the partial derivatives of $\Phi$ with respect to first and second variables. Since $(D \Phi)_{(0,0)}=\left(D^{2} \operatorname{Exp}\right)_{(0,0)}=\mathrm{id}_{E}$, as $\left(D^{2} \exp \right)_{0,0}$ $=\mathrm{id}_{\mathbb{E}}$, and $\exp : \mathfrak{g} \rightarrow G$ is analytic we have the pointwise bound

$$
\left|\nabla_{A}^{2} u\right| \leq c\left(|\chi|+\left|\nabla_{A} \chi\right|\right)\left|\nabla_{A} \chi\right|+c(1+|\chi|)\left|\nabla_{A}^{2} \chi\right|,
$$

giving the second assertion. Similarly, as $* \Phi(\chi, \zeta)=\Phi(\chi, * \zeta)$ and $\nabla_{A}^{*} \nabla_{A} u=$ $-* \nabla_{A} * \nabla_{A} u$, we have

$$
\left|\nabla_{A}^{*} \nabla_{A} u\right| \leq c\left(|\chi|+\left|\nabla_{A} \chi\right|\right)\left|\nabla_{A} \chi\right|+c(1+|\chi|)\left|\nabla_{A}^{*} \nabla_{A} \chi\right|,
$$


giving the third assertion.

The preceding pointwise bounds for $\nabla_{A} u, \nabla_{A}^{2} u$, and $\nabla_{A}^{*} \nabla_{A} u$ yield the following estimates for the exponential map:

Lemma 7.2. Continue the hypotheses of Lemma 7.1. If $k \geq 2$ is an integer $\left(\right.$ so $\left.L_{k+1}^{2} \subset C^{0}\right), A$ is an $L_{k}^{2}$ connection on a $G$ bundle $E$, and $\chi \in L_{k+1}^{2}\left(\mathfrak{g}_{E}\right)$, then $e^{\chi} \in \mathcal{G}_{E}^{k+1}$ satisfies

$$
\begin{array}{r}
\left\|\nabla_{A} e^{\chi}\right\|_{L^{2}(X)} \leq\left\|\nabla_{A} \chi\right\|_{L^{2}(X)}+c\|\chi\|_{C^{0}(X)}\left\|\nabla_{A} \chi\right\|_{L^{2}(X)}, \\
\left\|\nabla_{A} e^{\chi}\right\|_{L^{\sharp}(X)} \leq\left\|\nabla_{A} \chi\right\|_{L^{\sharp}(X)}+c\|\chi\|_{C^{0}(X)}\left\|\nabla_{A} \chi\right\|_{L^{\sharp}(X)}, \\
\left\|\nabla_{A}^{2} e^{\chi}\right\|_{L^{2}(X)} \leq c\|\chi\|_{C^{0}(X)}\left\|\nabla_{A} \chi\right\|_{L^{2}(X)}+\left\|\nabla_{A} \chi\right\|_{L^{4}(X)}^{2} \\
+c\left(\mid 1+\|\chi\|_{C^{0}(X)}\right)\left\|\nabla_{A}^{2} \chi\right\|_{L^{2}(X)}, \\
\left\|\nabla_{A}^{*} \nabla_{A} e^{\chi}\right\|_{L^{\sharp}(X)} \leq c\|\chi\|_{C^{0}(X)}\left\|\nabla_{A} \chi\right\|_{L^{\sharp}(X)}+\left\|\nabla_{A} \chi\right\|_{L^{2 \sharp}(X)}^{2} \\
+c\left(\mid 1+\|\chi\|_{C^{0}(X)}\right)\left\|\nabla_{A}^{*} \nabla_{A} \chi\right\|_{L^{\sharp}(X)} .
\end{array}
$$

The bounds (1)-(4) continue to hold for $\chi \in L_{2}^{\sharp, 2}\left(\mathfrak{g}_{E}\right) \subset C^{0}\left(\mathfrak{g}_{E}\right)$, with $A$ an $L_{1}^{\sharp, 2}$ connection on $E$, and $\operatorname{Exp}: \Omega^{0}\left(\mathfrak{g}_{E}\right) \rightarrow \mathcal{G}_{E}$ extends to a continuous map $\operatorname{Exp}: L_{2}^{\sharp, 2}\left(\mathfrak{g}_{E}\right) \rightarrow \mathcal{G}_{E}^{2, \sharp, 2}$.

Let $\mathcal{A}_{E}^{1, \sharp, 2}=A_{0}+L_{1, A_{0}}^{\sharp, 2}\left(\Lambda^{1} \otimes \mathfrak{g}_{E}\right)$, for any $C^{\infty}$ reference connection $A_{0}$ on $E$. Recall that we have an embedding $L_{2}^{\sharp, 2}\left(\mathfrak{g}_{E}\right) \subset C^{0}\left(\mathfrak{g}_{E}\right)$ and that the space $L_{2}^{\sharp, 2}\left(\mathfrak{g}_{E}\right)$ is an algebra, while $L_{1}^{\sharp, 2}\left(\Lambda^{1} \otimes \mathfrak{g}_{E}\right)$ and $L_{1}^{2}\left(\Lambda^{1} \otimes \mathfrak{g}_{E}\right)$ are $L_{2}^{\sharp, 2}\left(\mathfrak{g}_{E}\right)$-modules. Therefore, the proofs of Propositions (A.2) and (A.3) in [10] extend to give the following analogue for $\mathcal{G}_{E}^{2, \sharp, 2}$ in place of $\mathcal{G}_{E}^{k+1}$ :

Lemma 7.3. Let $X$ be a closed Riemannian four-manifold and let $E$ be a Hermitian vector bundle over $X$. Then the following hold.

(1) The space $\mathcal{G}_{E}^{2, \sharp, 2}$ is a Banach Lie group with Lie algebra $T_{\mathrm{id}_{E}} \mathcal{G}_{E}^{2, \sharp, 2}=$ $L_{2}^{\sharp, 2}\left(\mathfrak{g}_{E}\right) ;$

(2) The action of $\mathcal{G}_{E}^{2, \sharp, 2}$ on $\mathcal{A}_{E}^{1}$ and $\mathcal{A}_{E}^{1, \sharp, 2}$ is smooth;

(3) For $A \in \mathcal{A}_{E}^{1, \sharp, 2}$, the differential, at the identity $\operatorname{id}_{E} \in \mathcal{G}_{E}^{2, \sharp, 2}$, of the map $\mathcal{G}_{E}^{2, \sharp, 2} \rightarrow \mathcal{A}_{E}^{1, \sharp, 2}$ given by $u \mapsto u(A)=A-\left(d_{A} u\right) u^{-1}$ is $\zeta \mapsto-d_{A} \zeta$ as a map $L_{2}^{2, \sharp}\left(\mathfrak{g}_{E}\right) \rightarrow L_{1}^{\sharp, 2}\left(\Lambda^{1} \otimes \mathfrak{g}_{E}\right)$, and similarly for $A \in \mathcal{A}_{E}^{1}$.

\section{Existence of gauge transformations via the inverse function theorem.}

Our goal in this section is to give an alternative, "direct" proof of Theorem 6.1 via the inverse function theorem. A direct argument-due to our overarching constraint that the constants given there ultimately depend at most on the $L^{2}$ norm of the curvature and the least positive eigenvalue 
$\nu_{0}\left[A_{0}\right]$ - appears to be difficult within the standard framework of $L_{2}^{p}(p>2)$ gauge transformations acting on $L_{1}^{p}$ connections; if this constraint is dropped then a direct proof is standard. However, we shall see that a direct argument is fairly straightforward within the framework of $L_{2}^{\sharp 2}$ gauge transformations.

We already know that $\pi\left(\mathbf{B}_{A_{0}}^{4}\left(\varepsilon_{0}\right)\right)$ is open in $\mathcal{B}_{E}^{k}$, so it necessarily contains an $L_{k, A_{0}}^{2}$-ball centered at $\left[A_{0}\right]$. Via the inverse function theorem we estimate the radii of $\mathcal{L}_{1, A_{0}}^{\sharp, 2}$ and $L_{1, A_{0}}^{\sharp, 2}$ balls, $B_{\left[A_{0}\right]}^{1, *, 2}(\varepsilon)$ and $B_{\left[A_{0}\right]}^{1, \sharp, 2}(\varepsilon)$, which are contained in $\pi\left(\mathbf{B}_{A_{0}}^{4}\left(\varepsilon_{0}\right)\right)$. Let us first dispose of the question of regularity for solutions to the second-order gauge-fixing equation:

Lemma 8.1. Let $X$ be a closed, Riemannian four-manifold. Then there is a constant $\varepsilon$ with the following significance. Let $G$ be compact Lie group and let $k \geq 2$ be an integer. Suppose that $A_{0}$ is an $L_{k}^{2}$ connection on a $G$ bundle $E$, that $a \in L_{k}^{2}\left(\Lambda^{1} \otimes \mathfrak{g}_{E}\right)$ and $\chi \in L_{2}^{\sharp}\left(\mathfrak{g}_{E}\right)$, and that $u=e^{\chi}$ is a solution to

$$
d_{A_{0}}^{*}\left(\left(d_{A_{0}} u\right) u^{-1}-u a u^{-1}\right)=0 .
$$

If $\left\|d_{A_{0}} u\right\|_{L^{4}}<\varepsilon$ then $\chi \in L_{k+1}^{2}\left(\mathfrak{g}_{E}\right)$ and $u=e^{\chi} \in \mathcal{G}_{E}^{k+1}$.

Proof. Differentiation and right multiplication by $u$ yields

$$
\begin{aligned}
& d_{A_{0}}^{*} d_{A_{0}} u+*\left(\left(* d_{A_{0}} u\right) \wedge u^{-1} d_{A_{0}} u\right)+*\left(d_{A_{0}} u \wedge * a\right)+u d_{A_{0}}^{*} a \\
& +*\left(u a \wedge * u^{-1} d_{A_{0}} u\right)=0 .
\end{aligned}
$$

From Lemma 4.2 we know that $u \in C^{0} \cap L_{1}^{2}$ and so the last four terms in (8.1) are in $L^{2}$. Hence, $d_{A_{0}}^{*} d_{A_{0}} u$ is in $L^{2}$ and so $u \in L_{2}^{2}$ by elliptic regularity for $d_{A_{0}}^{*} d_{A_{0}}$. The Sobolev embedding $L_{1}^{2} \subset L^{4}$ and multiplication $L^{4} \times L^{q} \rightarrow L^{p}$ for $2 \leq p<4$ and $1 / p=1 / 4+1 / q$ (so $4 \leq q<\infty$ ) now show that the last three terms in (8.1) are in $L^{p}$, so the equation takes the simpler form

$$
d_{A_{0}}^{*} d_{A_{0}} u+*\left(\left(* d_{A_{0}} u\right) \wedge u^{-1} d_{A_{0}} u\right)=v,
$$

where $v \in L^{p}\left(\mathfrak{g}_{E}\right)$ is the tautologically defined right-hand side and $u \in$ $L^{\infty} \cap L_{2}^{2}$. Setting $b=d_{A_{0}} u$ and noting that $d_{A_{0}} b=F_{A_{0}} u$, with $F_{A_{0}} \in$ $L_{k-1}^{2}\left(\Lambda^{2} \otimes \mathfrak{g}_{E}\right) \subset L_{1}^{2}\left(\Lambda^{2} \otimes \mathfrak{g}_{E}\right)$ and $F_{A_{0}} u \in L_{1}^{2}\left(\Lambda^{2} \otimes \mathfrak{g}_{E}\right)$. Thus, we may conveniently rewrite (8.2) as a first-order elliptic equation in $b \in L_{1}^{2}\left(\Lambda^{1} \otimes \mathfrak{g}_{E}\right)$,

$$
\left(d_{A_{0}}^{*}+d_{A_{0}}\right) b+*\left((* b) \wedge u^{-1} b\right)=v^{\prime} \in L^{p}\left(\mathfrak{g}_{E}\right) \oplus L^{p}\left(\Lambda^{2} \otimes \mathfrak{g}_{E}\right),
$$

where $2<p<4$ and $v^{\prime}=F_{A_{0}} u+v$. Finally, (8.3) can be rewritten as a local equation by writing $A_{0}=\Gamma+a_{0}$, where $\Gamma$ is the product connection in a local trivialization for $E$ over a small ball $U \subset X$. Thus, the operator $d_{A_{0}}^{*}+d_{A_{0}}$ is replaced by $d^{*}+d$ in (8.2) and the additional terms are absorbed into the $L^{p}$ inhomogeneous term $v^{\prime}$ to give:

$$
\left(d^{*}+d\right) b+*\left((* b) \wedge u^{-1} b\right)=v^{\prime \prime} \in L^{p}\left(U, \mathfrak{g}_{E}\right) \oplus L^{p}\left(U, \Lambda^{2} \otimes \mathfrak{g}_{E}\right) .
$$


This is a first-order, elliptic equation with a quadratic non-linearity and Proposition 3.10 in [6] implies that the solution $b=d_{A_{0}} u \in L_{1}^{2}\left(U, \Lambda^{1} \otimes \mathfrak{g}_{E}\right)$ is necessarily in $L_{1}^{p}\left(U^{\prime}, \Lambda^{1} \otimes \mathfrak{g}_{E}\right)$ for $U^{\prime} \Subset U$, provided $\|b\|_{L^{4}(U)}<\varepsilon(g, p, U)$, and so $u \in L_{2}^{p}\left(U^{\prime}, \mathfrak{g}_{E}\right)$. In particular, we find that $b \in L_{1}^{p}\left(X, \Lambda^{1} \otimes \mathfrak{g}_{E}\right)$ and $u \in L_{2}^{p}\left(X, \mathfrak{g}_{E}\right)$ for any $2<p<4$, provided $\left\|d_{A_{0}} u\right\|_{L^{4}}<\varepsilon(g, p, X)$. The bootstrapping argument of Proposition 3.3 in [6] now implies that $d_{A_{0}} u \in$ $L_{k}^{2}\left(X, \Lambda^{1} \otimes \mathfrak{g}_{E}\right)$. Thus $u \in \mathcal{G}_{E}^{k+1}$ and $\chi \in L_{k+1}^{2}\left(X, \mathfrak{g}_{E}\right)$, as desired.

We can now proceed to the main argument:

Theorem 8.2. Let $X$ be a closed, Riemannian four-manifold and let $G$ be compact Lie group. Then there are positive constants $c, z$ with the following significance. Let $E$ be a $G$ bundle over $X$ and suppose that that $A_{0} \in \mathcal{A}_{E}^{2}$, let $K_{0}\left[A_{0}\right]=\left(1+\nu_{0}\left[A_{0}\right]^{-1}\right)\left(1+\left\|F_{A_{0}}\right\|_{L^{2}}\right)$ and let $\varepsilon_{1}$ be a constant satisfying

$$
0<\varepsilon_{1} \leq z K_{0}^{-2} \text {. }
$$

If $A \in \mathcal{A}_{E}^{2}$ obeys $\left\|A-A_{0}\right\|_{L_{1, A_{0}}^{\sharp, 2}}<\varepsilon_{1}$ then $u \in \mathcal{G}_{E}^{3}$ exists such that

- $d_{A_{0}}^{*}\left(u(A)-A_{0}\right)=0$;

- $\left\|u(A)-A_{0}\right\|_{L_{1, A_{0}}^{2}} \leq c K_{0}\left\|A-A_{0}\right\|_{L_{1, A_{0}}^{\sharp, 2}}$;

- $\left\|u-\operatorname{id}_{E}\right\|_{L_{2, A_{0}}^{\sharp, 2}}<c K_{0}\left\|A-A_{0}\right\|_{L_{1, A_{0}}^{\sharp, 2}}$.

Proof. The argument is broadly similar to that of Lemma 3.6, except that we can show $\boldsymbol{\Psi}$ is a diffeomorphism directly — rather than just a local diffeomorphism - using the slightly stronger norms now at our disposal. Moreover, on this occasion we seek precise bounds on the solutions so we keep track of the dependence of constants on the curvature $F_{A_{0}}$ and the least positive eigenvalue $\nu_{0}=\nu_{0}\left[A_{0}\right]$ of the Laplacian $\Delta_{A_{0}}=d_{A_{0}}^{*} d_{A_{0}}$.

Write $A=A_{0}+a$ and observe that

$$
u(A)-A_{0}=A-A_{0}-\left(d_{A} u\right) u^{-1}=u a u^{-1}-\left(d_{A_{0}} u\right) u^{-1} .
$$

Recall that we have an $L^{2}$-orthogonal decomposition

$$
\Omega^{0}\left(\mathfrak{g}_{E}\right)=\left(\operatorname{Ker} d_{A_{0}}\right)^{\perp} \oplus \operatorname{Ker} d_{A_{0}}=\operatorname{Im} d_{A_{0}}^{*} \oplus \operatorname{Ker} d_{A_{0}},
$$

and that $d_{A_{0}}^{*}: L_{1}^{2}\left(\Lambda^{1} \otimes \mathfrak{g}_{E}\right) \rightarrow L^{2}\left(\mathfrak{g}_{E}\right)$ has closed range; this gives

$$
\begin{aligned}
L_{2 ; A_{0}}^{\sharp, 2}\left(\mathfrak{g}_{E}\right) & =\left.\left(\left.\operatorname{Ker} d_{A_{0}}\right|_{L_{2 ; A_{0}}^{\sharp, 2}}\right)^{\perp} \oplus \operatorname{Ker} d_{A_{0}}\right|_{L_{2 ; A_{0}}^{\sharp, 2}} \\
& =\left(\left.\operatorname{Ker} d_{A_{0}}^{*}\right|_{L_{2 ; A_{0}}^{\sharp, 2}}\right)^{\perp} \oplus\left(\left.\operatorname{Im} d_{A_{0}}^{*}\right|_{L_{1, A_{0}}^{\sharp, 2}}\right) .
\end{aligned}
$$

We have a similar $L^{2}$-orthogonal decomposition

$$
\Omega^{1}\left(\mathfrak{g}_{E}\right)=\operatorname{Im} d_{A_{0}} \oplus \operatorname{Ker} d_{A_{0}}^{*}=\left(\operatorname{Ker} d_{A_{0}}^{*}\right)^{\perp} \oplus \operatorname{Ker} d_{A_{0}}^{*},
$$


and $d_{A_{0}}: L_{1}^{2}\left(\mathfrak{g}_{E}\right) \rightarrow L^{2}\left(\Lambda^{1} \otimes \mathfrak{g}_{E}\right)$ has closed range; this leads to the $L^{2}$ orthogonal decomposition

$$
\begin{aligned}
L_{1, A_{0}}^{\sharp, 2}\left(\Lambda^{1} \otimes \mathfrak{g}_{E}\right) & =\left(\left.\operatorname{Im} d_{A_{0}}\right|_{L_{2 ; A_{0}}^{\sharp, 2}}\right) \oplus\left(\left.\operatorname{Ker} d_{A_{0}}^{*}\right|_{L_{1, A_{0}}^{\sharp, 2}}\right) \\
& =\left(\left.\operatorname{Ker} d_{A_{0}}^{*}\right|_{L_{2 ; A_{0}}^{\sharp, 2}}\right)^{\perp} \oplus\left(\left.\operatorname{Ker} d_{A_{0}}^{*}\right|_{L_{1, A_{0}}^{\sharp, 2}}\right) .
\end{aligned}
$$

We now define a map

$$
\begin{aligned}
& \Psi:\left(\operatorname{Ker}\left(\left.d_{A_{0}}\right|_{L_{2}^{\sharp, 2}}\right)\right)^{\perp} \oplus \operatorname{Ker}\left(\left.d_{A_{0}}^{*}\right|_{L_{1}^{\sharp, 2}}\right) \rightarrow L_{1, A_{0}}^{\sharp, 2}\left(\Lambda^{1} \otimes \mathfrak{g}_{E}\right), \\
& (\chi, a) \mapsto u a u^{-1}-\left(d_{A_{0}} u\right) u^{-1},
\end{aligned}
$$

where $u=e^{\chi}$ and the differential at $(\chi, a)$ given by

$$
\begin{gathered}
(D \Psi)_{(\chi, a)}:\left(\operatorname{Ker}\left(\left.d_{A_{0}}\right|_{L_{2}^{\sharp, 2}}\right)\right)^{\perp} \oplus \operatorname{Ker}\left(\left.d_{A_{0}}^{*}\right|_{L_{1}^{\sharp, 2}}\right) \rightarrow L_{1}^{\sharp, 2}\left(\Lambda^{1} \otimes \mathfrak{g}_{E}\right), \\
(\zeta, b) \mapsto u\left(-d_{A} \oplus \iota\right) u^{-1}(\zeta, b)=u\left(-d_{A} \zeta+b\right) u^{-1},
\end{gathered}
$$

since $(D \boldsymbol{\Psi})_{(0, a)}(\zeta, b)=-d_{A} \zeta+b$ and $\boldsymbol{\Psi}$ is $\mathcal{G}_{E}$-equivariant. Moreover, we have

$$
\left(D^{2} \boldsymbol{\Psi}\right)_{(\chi, a)}((\zeta, b),(\eta, \alpha))=u\left[\eta,-d_{A} \zeta+b\right] u^{-1}+u[\alpha, \zeta] u^{-1}
$$

for $(\zeta, b),(\eta, \alpha) \in\left(\operatorname{Ker}\left(\left.d_{A_{0}}\right|_{L_{2}^{\sharp, 2}}\right)\right)^{\perp} \oplus \operatorname{Ker}\left(\left.d_{A_{0}}^{*}\right|_{L_{1}^{\sharp, 2}}\right)$.

We now verify that the conditions of the inverse function theorem (Theorem 3.2) hold for suitable constants $K$ and $\delta$. The operator

$$
d_{A_{0}}:\left(\operatorname{Ker}\left(\left.d_{A_{0}}\right|_{L_{2}^{\sharp, 2}}\right)\right)^{\perp} \rightarrow\left(\operatorname{Ker}\left(\left.d_{A_{0}}^{*}\right|_{L_{1}^{\sharp, 2}}\right)\right)^{\perp}
$$

has a two-sided inverse

$$
G_{A_{0}}^{0} d_{A_{0}}^{*}:\left(\operatorname{Ker}\left(\left.d_{A_{0}}^{*}\right|_{L_{1}^{\sharp, 2}}\right)\right)^{\perp} \rightarrow\left(\operatorname{Ker}\left(\left.d_{A_{0}}\right|_{L_{2}^{\sharp, 2}}\right)\right)^{\perp} .
$$

Indeed, for $b \in\left(\operatorname{Ker}\left(\left.d_{A_{0}}^{*}\right|_{L_{1}^{\sharp, 2}}\right)\right)^{\perp}$, we have

$$
\left\|G_{A_{0}}^{0} d_{A_{0}}^{*} b\right\|_{L_{2, A_{0}}^{\sharp, 2}} \leq c_{0} K_{0}\left\|d_{A_{0}}^{*} b\right\|_{L^{\sharp, 2}} \leq c_{0} K_{0}\|b\|_{L_{1, A_{0}}^{\sharp, 2}},
$$

and so $G_{A_{0}}^{0} d_{A_{0}}^{*}$ has Hom $\left(L_{1, A_{0}}^{\sharp, 2}, L_{2, A_{0}}^{\sharp, 2}\right)$ operator norm bound

$$
\left\|G_{A_{0}}^{0} d_{A_{0}}^{*}\right\| \leq c_{0} K_{0} \text {. }
$$

In particular, we see that $(D \boldsymbol{\Psi})_{(0,0)}^{-1}=G_{A_{0}}^{0} d_{A_{0}}^{*} \oplus$ id satisfies

$$
\left\|(D \Psi)_{(0,0)}^{-1}\right\| \leq c_{0} K_{0}
$$

the first of the conditions we need to verify for $(D \boldsymbol{\Psi})_{(0,0)}$ in order to apply the inverse function theorem. 
It remains to compare $(D \Psi)_{(\chi, a)}$ and $(D \Psi)_{(0,0)}$ using the mean value theorem,

$(8.9) \quad(D \boldsymbol{\Psi})_{(\chi, a)}(\zeta, b)-(D \boldsymbol{\Psi})_{(0,0)}(\zeta, b)=\int_{0}^{1}\left(D^{2} \boldsymbol{\Psi}\right)_{(t \chi, t a)}((\zeta, b),(\chi, a)) d t$.

Thus, we need an estimate for $D^{2} \mathbf{\Psi}$ :

Claim 8.3. There is a universal polynomial function $f(x, y)$, depending only on $(X, g)$ and $G$, with $f(0,0)=0$, such that the following holds. For any $t \in[0,1]$ we have:

$$
\begin{aligned}
& \left\|\left(D^{2} \boldsymbol{\Psi}\right)_{(t \chi, t a)}((\zeta, b),(\chi, a))\right\|_{L_{1, A_{0}}^{\sharp, 2}} \\
& \leq f\left(\|\chi\|_{L_{2, A_{0}}^{\sharp, 2}},\|a\|_{L_{1, A_{0}}^{\sharp, 2}}\right)\left(\|\zeta\|_{L_{2, A_{0}}^{\sharp, 2}}+\|b\|_{L_{1, A_{0}}^{\sharp, 2}}\right) .
\end{aligned}
$$

Proof. From (8.7) we have the $L^{\sharp, 2}$ estimate

$$
\begin{aligned}
& \left\|\left(D^{2} \boldsymbol{\Psi}\right)_{(t \chi, t a)}((\zeta, b),(\chi, a))\right\|_{L^{\sharp, 2}} \\
& \quad \leq c\|\chi\|_{C^{0}}\left(\left\|d_{A_{0}} \zeta\right\|_{L^{\sharp, 2}}+\|a\|_{L^{\sharp, 2}}\|\zeta\|_{C^{0}}+\|b\|_{L^{\sharp, 2}}\right)+c\|a\|_{L^{\sharp, 2}}\|\zeta\|_{C^{0}},
\end{aligned}
$$

and thus:

$$
\begin{aligned}
& \left\|\left(D^{2} \boldsymbol{\Psi}\right)_{(t \chi, t a)}((\zeta, b),(\chi, a))\right\|_{L^{\sharp, 2}} \\
& \leq c\left(\|\chi\|_{L_{2, A_{0}}^{\sharp, 2}}+\|a\|_{L^{\sharp, 2}}\|\chi\|_{L_{2, A_{0}}^{\sharp, 2}}+\|a\|_{L^{\sharp, 2}}\right)\left(\|\zeta\|_{L_{2, A_{0}}^{\sharp, 2}}+\|b\|_{L^{\sharp, 2}}\right) .
\end{aligned}
$$

The $L^{2}$ estimate of $\nabla_{A_{0}}\left(D^{2} \boldsymbol{\Psi}\right)_{(t \chi, t a)}((\zeta, b),(\chi, a))$ is given by

$$
\begin{aligned}
& \left\|\nabla_{A_{0}}\left(D^{2} \mathbf{\Psi}\right)_{(t \chi, t a)}((\zeta, b),(\chi, a))\right\|_{L^{2}} \\
& \leq c\left(\left\|\nabla_{A_{0}} u\right\|_{L^{4}}\|\chi\|_{C^{0}}+\left\|\nabla_{A_{0}} \chi\right\|_{L^{4}}\right)\left(\left\|d_{A_{0}} \zeta\right\|_{L^{4}}+\|a\|_{L^{4}}\|\zeta\|_{C^{0}}+\|b\|_{L^{4}}\right) \\
& \quad+c\|\chi\|_{C^{0}}\left(\left\|\nabla_{A_{0}}^{2} \zeta\right\|_{L^{2}}+\left\|\nabla_{A_{0}} a\right\|_{L^{2}}\|\zeta\|_{C^{0}}+\|a\|_{L^{4}}\left\|\nabla_{A_{0}} \zeta\right\|_{L^{4}}+\left\|\nabla_{A_{0}} b\right\|_{L^{2}}\right) \\
& \quad+c\left\|\nabla_{A_{0}} u\right\|_{L^{4}}\|a\|_{L^{4}}\|\zeta\|_{L^{4}}+c\left\|\nabla_{A_{0}} a\right\|_{L^{2}}\|\zeta\|_{C^{0}}+c\|a\|_{L^{4}}\left\|\nabla_{A_{0}} \zeta\right\|_{L^{4}},
\end{aligned}
$$

and hence, using Lemma 7.2 to estimate $u=e^{\chi}$ in terms of $\chi$,

$$
\begin{aligned}
& \left\|\nabla_{A_{0}}\left(D^{2} \boldsymbol{\Psi}\right)_{(t \chi, t a)}((\zeta, b),(\chi, a))\right\|_{L^{2}} \\
& \quad \leq f_{1}\left(\|\chi\|_{L_{2, A_{0}}^{\sharp, 2}},\|a\|_{L_{1, A_{0}}^{2}}\right)\left(\|\zeta\|_{L_{2, A_{0}}^{\sharp, 2}}+\|b\|_{L_{1, A_{0}}^{2}}\right),
\end{aligned}
$$

where $f_{1}(x, y)$ is a polynomial function with $f_{1}(0,0)=0$.

Noting that $d_{A_{0}}^{*} a=0$, we have

$$
\begin{aligned}
d_{A_{0}}^{*}[a, \zeta] & =d_{A_{0}}^{*}(a \zeta-\zeta a) \\
& =\left(d_{A_{0}}^{*} a\right) \zeta-a \wedge d_{A_{0}} \zeta-*\left(d_{A_{0}} \zeta \wedge * a\right)-\zeta\left(d_{A_{0}}^{*} a\right) \\
& =-a \wedge d_{A_{0}} \zeta-*\left(d_{A_{0}} \zeta \wedge * a\right),
\end{aligned}
$$


and similarly for $d_{A_{0}}^{*}[\chi, b]$ since $d_{A_{0}}^{*} b=0$. For any $\beta \in L_{1}^{2}\left(\Lambda^{1} \otimes \mathfrak{g}_{E}\right)$ we have $(8.13)$

$$
\begin{aligned}
d_{A_{0}}^{*}\left(u \beta u^{-1}\right)= & \left.-* d_{A_{0}}\left(u(* \beta) u^{-1}\right)\right) \\
= & -*\left(d_{A_{0}} u \wedge * \beta u^{-1}\right)+u\left(d_{A_{0}}^{*} \beta\right) u^{-1} \\
& -* u\left((* \beta) \wedge u\left(d_{A_{0}} u\right) u^{-1}\right) .
\end{aligned}
$$

Therefore, Equations (8.7), (8.12), and (8.13) and the estimates for $u=e^{\chi}$ in Lemma 7.2 yield

$$
\begin{aligned}
\| d_{A_{0}}^{*} & \left(D^{2} \boldsymbol{\Psi}\right)_{(\chi, a)}((\zeta, b),(\chi, a)) \|_{L^{\sharp, 2}} \\
& \leq\left\|d_{A_{0}}^{*}\left(u\left[\chi,-d_{A} \zeta+b\right] u^{-1}+u[a, \zeta] u^{-1}\right)\right\|_{L^{\sharp, 2}} \\
& \leq f_{2}\left(\|\chi\|_{L_{2, A_{0}}^{\sharp, 2}},\|a\|_{L^{2 \sharp, 4}}\right)\left(\|\zeta\|_{L_{2, A_{0}}^{\sharp, 2}}+\|b\|_{L^{2 \sharp, 4}}\right),
\end{aligned}
$$

where $f_{2}(x, y)$ is a polynomial function with $f_{2}(0,0)=0$. The claim now follows by combining (8.10), (8.11), and (8.14).

Therefore, from Claim 8.3 and (8.9) we have

$$
\begin{aligned}
& \left\|(D \Psi)_{(\chi, a)}(\zeta, b)-(D \Psi)_{(0,0)}(\zeta, b)\right\|_{L_{1, A_{0}}^{\sharp, 2}} \\
& \leq f\left(\|\chi\|_{L_{2, A_{0}}^{\sharp, 2}},\|a\|_{L_{1, A_{0}}^{\sharp, 2}}\right)\left(\|\zeta\|_{L_{2, A_{0}}^{\sharp, 2}}+\|b\|_{L_{1, A_{0}}^{\sharp, 2}}\right) .
\end{aligned}
$$

Consequently, with respect to the Hom $\left(L_{2, A_{0}}^{\sharp, 2}, L_{1, A_{0}}^{\sharp, 2}\right)$ operator norm, (8.15) yields the bound

$$
\left\|(D \boldsymbol{\Psi})_{(\chi, a)}-(D \boldsymbol{\Psi})_{(0,0)}\right\| \leq \frac{1}{2} c_{0}^{-1} K_{0}^{-1},
$$

where $c_{0} K_{0}=K$ is the constant of $(8.8)$, provided $(\chi, a)$ satisfies the constraint

$$
\|\chi\|_{L_{2, A_{0}}^{\sharp, 2}}+\|a\|_{L_{1, A_{0}}^{\sharp, 2}} \leq c_{1} K_{0}^{-1}=\delta .
$$

Define balls centered at the origins in $\left(\operatorname{Ker}\left(\left.d_{A_{0}}\right|_{L_{2}^{\sharp, 2}}\right)\right)^{\perp}$ and $\operatorname{Ker}\left(\left.d_{A_{0}}^{*}\right|_{L_{1}^{\sharp, 2}}\right)$ by setting

$$
\begin{aligned}
B_{0}^{\perp ; 2, \sharp, 2}(\delta) & =\left\{\chi \in\left(\operatorname{Ker}\left(\left.d_{A_{0}}\right|_{L_{2}^{\sharp, 2}}\right)\right)^{\perp}:\|\chi\|_{L_{2, A_{0}}^{\sharp, 2}}<\delta\right\}, \\
\mathbf{B}_{0}^{1, \sharp, 2}(\delta) & =\left\{a \in \operatorname{Ker}\left(\left.d_{A_{0}}^{*}\right|_{L_{1}^{\sharp, 2}}\right):\|a\|_{L_{1, A_{0}}^{\sharp, 2}}<\delta\right\} .
\end{aligned}
$$

Hence, Theorem 3.2 implies that the map

$$
\mathbf{\Psi}: B_{0}^{\perp ; 2, \sharp, 2}(\delta) \times \mathbf{B}_{0}^{1, \sharp, 2}(\delta) \rightarrow \mathcal{A}_{E}^{1, \sharp, 2}
$$

is injective, its image is an open subset of $\mathcal{A}_{E}^{1, \sharp, 2}$ and contains the ball $B_{A_{0}}^{1, \sharp, 2}(\delta /(2 K))$, the inverse map $\Psi^{-1}$ is a diffeomorphism from $B_{A_{0}}^{1, \sharp, 2}(\delta /(2 K))$ 
onto its image, and if $\left(\chi_{1}, A_{1}\right),\left(\chi_{2}, A_{2}\right)$ are points in $B_{0}^{\perp, 2, \sharp, 2}(\delta) \times \mathbf{B}_{0}^{1, \sharp, 2}(\delta)$, then

$$
\left\|\chi_{1}-\chi_{2}\right\|_{L_{2, A_{0}}^{\sharp, 2}}+\left\|A_{1}-A_{2}\right\|_{L_{1, A_{0}}^{\sharp, 2}} \leq 2 K\left\|u_{1}\left(A_{1}\right)-u_{2}\left(A_{2}\right)\right\|_{L_{1, A_{0}}^{\sharp, 2}},
$$

where $u_{i}=e^{\chi_{i}}, i=1,2$. In particular, setting $\left(\chi_{2}, A_{2}-A_{0}\right)=(0,0)$, we see that if $A$ is a point in $\mathcal{A}_{E}^{1, \sharp, 2}$ such that $\left\|A-A_{0}\right\|_{L_{1, A_{0}}^{\sharp, 2}}<\delta /(2 K)$, then there is a unique solution $\left(\chi, u^{-1}(A)\right)=\mathbf{\Psi}^{-1}(A)$ in $B_{0}^{\perp, 2, \sharp, 2}(\delta) \times \mathbf{B}_{0}^{1, \sharp, 2}(\delta)$. Here, $u=e^{\chi}$ is a gauge transformation with $\chi \in B_{0}^{\perp ; 2, \sharp, 2}(\delta)$ such that

$$
\begin{aligned}
d_{A_{0}}^{*}\left(u^{-1}(A)-A_{0}\right) & =0, \\
\|\chi\|_{L_{2, A_{0}}^{\sharp, 2}}+\left\|u^{-1}(A)-A_{0}\right\|_{L_{1, A_{0}}^{\sharp, 2}} & \leq 2 K\left\|A-A_{0}\right\| L_{1, A_{0}}^{\sharp, 2} .
\end{aligned}
$$

Lemma 7.2 implies that $u=e^{\chi}$ satisfies

$$
\left\|u-\mathrm{id}_{E}\right\|_{L_{2, A_{0}}^{\sharp, 2}} \leq f_{3}\left(\|\chi\|_{L_{2, A_{0}}^{\sharp, 2}}\right) \leq c\|\chi\|_{L_{2, A_{0}}^{\sharp, 2}} \leq c_{2} \delta,
$$

where $f_{3}(x)$ is a polynomial with coefficients depending only on $(X, g)$ and $G$ such that $f_{3}(0)=0$. Noting that $K=c_{0} K_{0}, \delta=c_{1} K_{0}^{-1}$, and $\delta /(2 K)=$ $\frac{1}{2} c_{0} c_{1} K_{0}^{-2}$, the desired estimates follows from (8.18) and (8.19). Finally, Lemma 8.1 implies that $u \in \mathcal{G}_{E}^{3}$ and this completes the proof of the theorem.

While the $L_{1}^{2}$ estimate of Theorem 8.2 suffices for most purposes, it is occasionally useful to have the weaker $L^{2 \sharp, 4}$ bound at hand. Recall from Section 4 that we defined

$$
\|a\|_{\mathcal{L}_{1, A_{0}}^{\sharp, 2}}=\|a\|_{L^{2 \sharp, 4}}+\left\|d_{A_{0}}^{*} a\right\|_{L^{\sharp, 2}}, \quad a \in \Omega^{1}\left(\mathfrak{g}_{E}\right) .
$$

A slight modification of the proof of Theorem 8.2 yields:

Theorem 8.4. Continue the hypotheses of Theorem 8.2. Then for any $A \in$ $\mathcal{A}_{E}^{2}$ such that $\left\|A-A_{0}\right\|_{\mathcal{L}_{1, A_{0}}^{\sharp, 2}}<\varepsilon_{1}$ there is a gauge transformation $u \in \mathcal{G}_{E}^{3}$ with the following properties:

- $d_{A_{0}}^{*}\left(u(A)-A_{0}\right)=0$;

- $\left\|u(A)-A_{0}\right\|_{L^{2 \sharp, 4}} \leq c K_{0}\left\|A-A_{0}\right\|_{\mathcal{L}_{1, A_{0}}^{\sharp, 2}} ;$

- $\left\|u-\operatorname{id}_{E}\right\|_{L_{2, A_{0}}^{\sharp, 2}}<c K_{0}\left\|A-A_{0}\right\|_{\mathcal{L}_{1, A_{0}}^{\sharp, 2}}$.

Proof. The first difference in the argument is that the map $\boldsymbol{\Psi}$ in (8.5) is replaced by

$$
\begin{aligned}
& \Psi:\left(\operatorname{Ker}\left(\left.d_{A_{0}}\right|_{L_{2}^{\sharp, 2}}\right)\right)^{\perp} \oplus \operatorname{Ker}\left(\left.d_{A_{0}}^{*}\right|_{\mathcal{L}_{1}^{\sharp, 2}}\right) \rightarrow \mathcal{L}_{1, A_{0}}^{\sharp, 2}\left(\Lambda^{1} \otimes \mathfrak{g}_{E}\right), \\
& \quad(\chi, a) \mapsto u a u^{-1}-\left(d_{A_{0}} u\right) u^{-1} .
\end{aligned}
$$

The second difference is that Claim 8.3 is replaced by: 
Claim 8.5. There is a universal polynomial function $f(x, y)$, depending only on $(X, g)$ and $G$, with $f(0,0)=0$, such that the following holds. For any $t \in[0,1]$ we have:

$$
\begin{aligned}
& \left\|\left(D^{2} \boldsymbol{\Psi}\right)_{(t \chi, t a)}((\zeta, b),(\chi, a))\right\|_{\mathcal{L}_{1, A_{0}}^{\sharp, 2}} \\
& \quad \leq f\left(\|\chi\|_{L_{2, A_{0}}^{\sharp, 2}},\|a\|_{L^{2 \sharp, 4}}\right)\left(\|\zeta\|_{L_{2, A_{0}}^{\sharp, 2}}+\|b\|_{L^{2 \sharp, 4}}\right) .
\end{aligned}
$$

Proof. From (8.7) we now have the $L^{2 \sharp, 4}$ estimate

$$
\begin{aligned}
& \left\|\left(D^{2} \boldsymbol{\Psi}\right)_{(t \chi, t a)}((\zeta, b),(\chi, a))\right\|_{L^{2 \sharp, 4}} \\
& \quad \leq c\|\chi\|_{C^{0}}\left(\left\|d_{A_{0}} \zeta\right\|_{L^{2 \sharp, 4}}+\|a\|_{L^{2 \sharp, 4}}\|\zeta\|_{C^{0}}+\|b\|_{L^{2 \sharp, 4}}\right)+c\|a\|_{L^{2 \sharp, 4}}\|\zeta\|_{C^{0}},
\end{aligned}
$$

and thus:

$$
\begin{aligned}
& \left\|\left(D^{2} \boldsymbol{\Psi}\right)_{(t \chi, t a)}((\zeta, b),(\chi, a))\right\|_{L^{2 \sharp, 4}} \\
& \leq c\left(\|\chi\|_{L_{2, A_{0}}^{\sharp, 2}}+\|a\|_{L^{2 \sharp, 4}}\|\chi\|_{L_{2, A_{0}}^{\sharp, 2}}+\|a\|_{L^{2 \sharp, 4}}\right) \\
& \quad \times\left(\|\zeta\|_{L_{2, A_{0}}^{\sharp, 2}}+\|b\|_{L^{2 \sharp, 4}}\right) .
\end{aligned}
$$

Combining (8.14) and (8.21) yields the claim.

The rest of the argument is just as before. This completes the proof of the theorem.

We now have our second proof of Theorem 6.1 via Theorems 8.2 and 8.4:

Proof of Theorem 6.1. From the hypotheses we have $A_{0} \in \mathcal{A}_{E}^{k}$ and $[A] \in \mathcal{B}_{E}^{k}$ with $k \geq 2$. According to Lemma 6.3, there is gauge transformation $w \in \mathcal{G}_{E}^{3}$ such that

$$
\operatorname{dist}_{L_{1, A_{0}}^{\sharp, 2}}\left([A],\left[A_{0}\right]\right)=\left\|w(A)-A_{0}\right\|_{L_{1, A_{0}}^{\sharp, 2}},
$$

where $A \in \mathcal{A}_{E}^{k}$, so Theorems 8.2 and the argument of 8.4 imply that there is a gauge transformation $v \in \mathcal{G}_{E}^{3}$ so that $u(A)$ satisfies the conclusions of Assertion (2) with $u=v w \in \mathcal{G}_{E}^{3}$. Since $d_{A_{0}}^{*}\left(u(A)-A_{0}\right)=0$ and $u \in \mathcal{G}_{E}^{3}$ and $A, A_{0} \in \mathcal{A}_{E}^{k}$, a standard bootstrapping argument implies that $u \in \mathcal{G}_{E}^{k+1}$.

Similarly, by Lemma 6.3, there is gauge transformation $w \in \mathcal{G}_{E}^{3}$ such that

$$
\operatorname{dist}_{\mathcal{L}_{1, A_{0}}^{\sharp, 2}}\left([A],\left[A_{0}\right]\right)=\left\|w(A)-A_{0}\right\|_{L_{1, A_{0}}^{\sharp, 2}},
$$

so Assertion (1) follows from Theorem 8.4 in the same manner. 


\section{References}

[1] R.A. Adams, Sobolev Spaces, Academic Press, Orlando, FL, 1975, MR 56 \#9247, Zbl 314.46.030.

[2] M.F. Atiyah, N.J. Hitchin and I.M. Singer, Self-duality in four-dimensional Riemannian geometry, Proc. Royal Soc. London, A 362 (1978), 425-461, MR 80d:53023, Zbl 389.53011.

[3] T. Aubin, Nonlinear Analysis on Complex Manifolds. Monge-Ampère Equations, Springer, New York, 1982, MR 85j:58002, Zbl 512.53044.

[4] S.K. Donaldson, The approximation of instantons, Geom. Funct. Anal., 3 (1993), 179-200, MR 94k:58030, Zbl 778.57010.

[5] S.K. Donaldson and P.B. Kronheimer, The Geometry of Four-Manifolds, Oxford Univ. Press, Oxford, 1990, MR 92a:57036, Zbl 820.57002.

[6] P.M.N. Feehan and T.G. Leness, PU(2) monopoles. I: Regularity, Uhlenbeck compactness, and transversality, J. Differential Geom., 49 (1998), 265-410, MR 2000e:57052.

[7] _ $P U(2)$ monopoles. II: Top-level Seiberg-Witten moduli spaces and Witten's conjecture in low degrees, J. Reine Angew. Math., to appear.

[8] — Donaldson invariants and wall-crossing formulas. I: Continuity of gluing and obstruction maps, submitted to a print journal.

[9] _ $P U(2)$ monopoles. III: Existence of gluing and obstruction maps, submitted to a print journal.

[10] D. Freed and K.K. Uhlenbeck, Instantons and Four-Manifolds, 2nd ed., Springer, New York, 1991, MR 91i:57019.

[11] H.B. Lawson, The Theory of Gauge Fields in Four Dimensions, Conf. Board Math. Sci., 58, Amer. Math. Soc., Providence, RI, 1985, MR 87d:58044, Zbl 597.53001.

[12] H.B. Lawson and M.-L. Michelsohn, Spin Geometry, Princeton Univ. Press, Princeton, NJ, 1988.

[13] P.K. Mitter and C.M. Viallet, On the bundle of connections and the gauge orbit manifold in Yang-Mills theory, Comm. Math. Phys., 79 (1981), 451-472, MR 83f:81056, Zbl 474.58004.

[14] J.W. Morgan, Gauge theory and the topology of smooth four-manifolds, Harvard University lecture notes, 1988.

[15] R.S. Palais, Foundations of Global Non-linear Analysis, Benjamin, New York, 1968, MR 40 \#2130, Zbl 164.11102.

[16] T.H. Parker, Gauge theories on four-dimensional Riemannian manifolds, Comm. Math. Phys., 85 (1982), 563-602, MR 84b:58036, Zbl 502.53022.

[17] T.H. Parker and C.H. Taubes, On Witten's proof of the positive energy theorem, Comm. Math. Phys., 84 (1982), 223-238, MR 83m:83020, Zbl 528.58040.

[18] I.M. Singer, Some remarks on the Gribov ambiguity, Comm. Math. Phys., 60 (1978), 7-12, MR 80d:53025, Zbl 379.53009.

[19] E. Stein, Singular Integral Operators and Differentiability Properties of Functions, Princeton Univ. Press, Princeton, NJ, 1970, Zbl 207.13501.

[20] C.H. Taubes, Self-dual Yang-Mills connections on non-self-dual 4-manifolds, J. Differential Geom., 17 (1982), 139-170, MR 83i:53055, Zbl 484.53026. 
[21] _ Path-connected Yang-Mills moduli spaces, J. Differential Geom., 19 (1984), 337-392, MR 85m:58049, Zbl 551.53040.

[22] _ Self-dual connections on 4-manifolds with indefinite intersection matrix, J. Differential Geom., 19 (1984), 517-560, MR 86b:53025, Zbl 552.53011.

[23] _ A framework for Morse theory for the Yang-Mills functional, Invent. Math., 94 (1988), 327-402, MR 90a:58035, Zbl 665.58006.

[24] _ The stable topology of self-dual moduli spaces, J. Differential Geom., 29 (1989), 162-230, MR 90f:58023, Zbl 669.58005.

[25] _ The existence of anti-self-dual conformal structures, J. Differential Geom., 36 (1992), 163-253, MR 93j:53063, Zbl 822.53006.

[26] K.K. Uhlenbeck, Connections with $L^{p}$ bounds on curvature, Comm. Math. Phys., 83 (1982), 31-42, MR 83e:53035, Zbl 499.58019.

Received July 29, 1999 and revised August 10, 2000. The author was supported in part by an NSF Mathematical Sciences Postdoctoral Fellowship under grant DMS 9306061 and by NSF grant DMS 9704174.

Rutgers University

PiscataWAy, NJ 08854-8019

E-mail address: feehan@math.rutgers.edu

UNIVERSITY OF DUBLIN

Trinity College

DUBLIN 2

IRELAND 